\title{
Dark energy: investigation and modeling
}

\author{
Shinji Tsujikawa ${ }^{1}$ \\ ${ }^{1}$ Department of Physics, Faculty of Science, Tokyo University of Science, \\ 1-3, Kagurazaka, Shinjuku-ku, Tokyo 162-8601, Japan
}

(Dated: April 12, 2010)

\begin{abstract}
Constantly accumulating observational data continue to confirm that about $70 \%$ of the energy density today consists of dark energy responsible for the accelerated expansion of the Universe. We present recent observational bounds on dark energy constrained by the type Ia supernovae, cosmic microwave background, and baryon acoustic oscillations. We review a number of theoretical approaches that have been adopted so far to explain the origin of dark energy. This includes the cosmological constant, modified matter models (such as quintessence, k-essence, coupled dark energy, unified models of dark energy and dark matter), modified gravity models (such as $f(R)$ gravity, scalar-tensor theories, braneworlds), and inhomogeneous models. We also discuss observational and experimental constraints on those models and clarify which models are favored or ruled out in current observations.
\end{abstract}

\section{Contents}

\section{Introduction}

II. Observational constraints on dark energy

A. Supernovae Ia observations

B. $\mathrm{CMB}$

C. $\mathrm{BAO}$

III. Cosmological constant

IV. Modified matter models $\quad 11$

A. Quintessence 11

B. k-essence

C. Coupled dark energy $\quad 17$

1. The coupling between dark energy and dark matter 17

2. Coupled dark energy and coincidence problem $\quad 19$

3. Chameleon mechanism $\quad 20$

4. Varying $\alpha+22$

D. Unified models of dark energy and dark matter 23

V. Modified gravity models $\quad 24$

A. $f(R)$ gravity 24

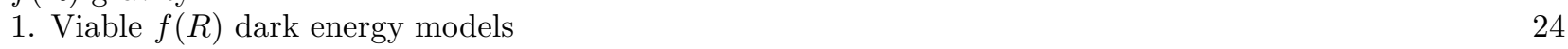

2. Observational signatures of $f(R)$ dark energy models 26

3. Local gravity constraints $\quad 28$

4. Palatini $f(R)$ gravity $\quad 29$

B. Gauss-Bonnet dark energy models $\quad 30$

C. Scalar-tensor theories $\quad 31$

D. DGP model

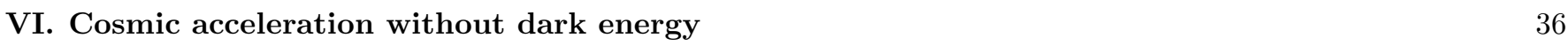

A. Inhomogeneous Lemaître-Tolman-Bondi model $\quad 36$

B. Backreaction of cosmological perturbations

\begin{tabular}{ll} 
VII. Conclusions & 38 \\
\hline
\end{tabular}

\begin{tabular}{ll} 
Acknowledgments & 39 \\
\hline
\end{tabular}

\begin{tabular}{ll} 
References & 39 \\
\hline
\end{tabular} 


\section{INTRODUCTION}

The discovery of the late-time cosmic acceleration reported in 1998 [1, 2] based on the type Ia Supernovae (SN Ia) observations opened up a new field of research in cosmology. The source for this acceleration, dubbed dark energy [3], has been still a mystery in spite of tremendous efforts to understand its origin over the last decade [4 12]. Dark energy is distinguished from ordinary matter in that it has a negative pressure whose equation of state $w_{\mathrm{DE}}$ is close to -1 . Independent observational data such as SN Ia [13 18], Cosmic Microwave Background (CMB) 19 22], and Baryon acoustic oscillations (BAO) [23 25] have continued to confirm that about $70 \%$ of the energy density of the present Universe consists of dark energy.

The simplest candidate for dark energy is the so-called cosmological constant $\Lambda$ whose equation of state is $w_{\mathrm{DE}}=-1$. If the cosmological constant originates from a vacuum energy of particle physics, its energy scale is significantly larger than the dark energy density today [26] $\left(\rho_{\mathrm{DE}}^{(0)} \simeq 10^{-47} \mathrm{GeV}^{4}\right)$. Hence we need to find a mechanism to obtain the tiny value of $\Lambda$ consistent with observations. A lot of efforts have been made in this direction under the framework of particle physics. For example, the recent development of string theory shows that it is possible to construct de Sitter vacua by compactifying extra dimensions in the presence of fluxes with an account of non-perturbative corrections [27].

The first step toward understanding the property of dark energy is to clarify whether it is a simple cosmological constant or it originates from other sources that dynamically change in time. The dynamical dark energy models can be distinguished from the cosmological constant by considering the evolution of $w_{\mathrm{DE}}$. The scalar field models of DE such as quintessence [28 38] and k-essence [39 41] predict a wide variety of variations of $w_{\mathrm{DE}}$, but still the current observational data are not sufficient to provide some preference of such models over the $\Lambda$-Cold-Dark-Matter $(\Lambda \mathrm{CDM})$ model. Moreover, the field potentials need to be sufficiently flat such that the field evolves slowly enough to drive the present cosmic acceleration. This demands that the field mass is extremely small $\left(m_{\phi} \simeq 10^{-33} \mathrm{eV}\right)$ relative to typical mass scales appearing in particle physics [42, 43]. However it is not entirely hopeless to construct viable scalar-field dark energy models in the framework of particle physics. We note that there is another class of modified matter models based on perfect fluids-so-called (generalized) the Chaplygin gas model [44, 45]. If these models are responsible for explaining the origin of dark matter as well as dark energy, then they are severely constrained from the matter power spectrum in galaxy clustering [46].

There exists another class of dynamical dark energy models that modify General Relativity. The models that belong to this class are $f(R)$ gravity [47, 49 52] ( $f$ is a function of the Ricci scalar $R$ ), scalar-tensor theories [53 57], and Dvali, Gabadadze and Porrati (DGP) braneworld model [58]. The attractive feature of these models is that the cosmic acceleration can be realized without recourse to a dark energy component. If we modify gravity from General Relativity, however, there are stringent constraints coming from local gravity tests as well as a number of observational constraints such as large-scale structure (LSS) and CMB. Hence the restriction on modified gravity models is in general very tight compared to modified matter models. We shall construct viable modified gravity models and discuss their observational and experimental signatures.

In addition to the above mentioned models, there are attempts to explain the cosmic acceleration without dark energy. One example is the void model in which an apparent accelerated expansion is induced by a large spatial inhomogeneity [59 63]. Another example is the so-called backreaction model in which the backreaction of spatial inhomogeneities on the Friedmann-Lemaittre-Robertson-Walker (FLRW) background is responsible for the real acceleration [64 66]. We shall discuss these models as well.

This review is organized as follows. In Sec. III we provide recent observational constraints on dark energy obtained by SN Ia, CMB, and BAO data. In Sec. III we review theoretical attempts to explain the origin of the cosmological constant consistent with the low energy scale of dark energy. In Sec. IV we discuss modified gravity models of dark energy-including quintessence, k-essence, coupled dark energy, and unified models of dark energy and dark matter. In Sec. $\mathrm{V}$ we review modified gravity models and provide a number of ways to distinguish those models observationally from the $\Lambda$ CDM model. Sec. VI is devoted to the discussion about the cosmic acceleration without using dark energy. We conclude in Sec. VII]

We use units such that $c=\hbar=1$, where $c$ is the speed of light and $\hbar$ is reduced Planck's constant. The gravitational constant $G$ is related to the Planck mass $m_{\mathrm{pl}}=1.2211 \times 10^{19} \mathrm{GeV}$ via $G=1 / m_{\mathrm{pl}}^{2}$ and the reduced Planck mass $M_{\mathrm{pl}}=2.4357 \times 10^{18} \mathrm{GeV}$ via $\kappa^{2} \equiv 8 \pi G=1 / M_{\mathrm{pl}}^{2}$, respectively. We write the Hubble constant today as $H_{0}=100 h \mathrm{~km} \mathrm{sec}^{-1} \mathrm{Mpc}^{-1}$, where $h$ describes the uncertainty on the value $H_{0}$. We use the metric signature $(-,+,+,+)$. 


\section{OBSERVATIONAL CONSTRAINTS ON DARK ENERGY}

The late-time cosmic acceleration is supported by a number of independent observations-such as (i) supernovae observations, (ii) Cosmic Microwave Background (CMB), and (iii) Baryon acoustic oscillations (BAO). In this section we discuss observational constraints on the property of dark energy.

\section{A. Supernovae Ia observations}

In 1998 Riess et al. [1] and Perlmutter et al. et al. [2] independently reported the late-time cosmic acceleration by observing distant supernovae of type Ia (SN Ia). The line-element describing a 4-dimensional homogeneous and isotropic Universe, which is called the FLRW space-time, is given by [67]

$$
\mathrm{d} s^{2}=g_{\mu \nu} \mathrm{d} x^{\mu} \mathrm{d} x^{\nu}=-\mathrm{d} t^{2}+a^{2}(t)\left[\frac{\mathrm{d} r^{2}}{1-K r^{2}}+r^{2}\left(\mathrm{~d} \theta^{2}+\sin ^{2} \theta \mathrm{d} \phi^{2}\right)\right],
$$

where $a(t)$ is the scale factor with cosmic time $t$, and $K=+1,-1,0$ correspond to closed, open and flat geometries, respectively. The redshift $z$ is defined by $z=a_{0} / a-1$, where $a_{0}=1$ is the scale factor today.

In order to discuss the cosmological evolution in the low-redshift regime $(z<\mathcal{O}(1))$, let us consider non-relativistic matter with energy density $\rho_{m}$ and dark energy with energy density $\rho_{\mathrm{DE}}$ and pressure $P_{\mathrm{DE}}$, satisfying the continuity equations

$$
\begin{aligned}
& \dot{\rho}_{m}+3 H \rho_{m}=0, \\
& \dot{\rho}_{\mathrm{DE}}+3 H\left(\rho_{\mathrm{DE}}+P_{\mathrm{DE}}\right)=0,
\end{aligned}
$$

which correspond to the conservation of the energy-momentum tensor $T_{\mu \nu}$ for each component $\left(\nabla_{\mu} T^{\mu \nu}=0\right.$, where $\nabla$ represents a covariant derivative). Note that a dot represents a derivative with respect to $t$. The cosmological dynamics is known by solving the Einstein equations

$$
G_{\mu \nu}=8 \pi G T_{\mu \nu}
$$

where $G_{\mu \nu}$ is the Einstein tensor. For the metric (1) the (00) component of the Einstein equations gives 67]

$$
H^{2}=\frac{8 \pi G}{3}\left(\rho_{m}+\rho_{\mathrm{DE}}\right)-\frac{K}{a^{2}}
$$

where $H \equiv \dot{a} / a$ is the Hubble parameter. We define the density parameters

$$
\Omega_{m} \equiv \frac{8 \pi G \rho_{m}}{3 H^{2}}, \quad \Omega_{\mathrm{DE}} \equiv \frac{8 \pi G \rho_{\mathrm{DE}}}{3 H^{2}}, \quad \Omega_{K} \equiv-\frac{K}{(a H)^{2}},
$$

which satisfy the relation $\Omega_{m}+\Omega_{\mathrm{DE}}+\Omega_{K}=1$ from Eq. (15). Integrating Eqs. (2) and (3), we obtain

$$
\rho_{m}=\rho_{m}^{(0)}(1+z)^{3}, \quad \rho_{\mathrm{DE}}=\rho_{\mathrm{DE}}^{(0)} \exp \left[\int_{0}^{z} \frac{3\left(1+w_{\mathrm{DE}}\right)}{1+\tilde{z}} \mathrm{~d} \tilde{z}\right]
$$

where " 0 " represents the values today and $w_{\mathrm{DE}}=P_{\mathrm{DE}} / \rho_{\mathrm{DE}}$ is the equation of state of dark energy. Plugging these relations into Eq. (5), it follows that

$$
H^{2}(z)=H_{0}^{2}\left[\Omega_{m}^{(0)}(1+z)^{3}+\Omega_{\mathrm{DE}}^{(0)} \exp \left\{\int_{0}^{z} \frac{3\left(1+w_{\mathrm{DE}}\right)}{1+\tilde{z}} \mathrm{~d} \tilde{z}\right\}+\Omega_{K}^{(0)}(1+z)^{2}\right] .
$$

The expansion rate $H(z)$ can be known observationally by measuring the luminosity distance $d_{L}(z)$ of SN Ia. The luminosity distance is defined by $d_{I}^{2} \equiv L_{s} /(4 \pi \mathcal{F})$, where $L_{s}$ is the absolute luminosity of a source and $\mathcal{F}$ is an observed flux. It is a textbook exercise [9, 12, 67] to derive $d_{L}(z)$ for the FLRW metric (11):

$$
d_{L}(z)=\frac{1+z}{H_{0} \sqrt{\Omega_{K}^{(0)}}} \sinh \left(\sqrt{\Omega_{K}^{(0)}} \int_{0}^{z} \frac{\mathrm{d} \tilde{z}}{E(\tilde{z})}\right)
$$


where $E(z) \equiv H(z) / H_{0}$. The function $f_{K}(\chi) \equiv 1 / \sqrt{\Omega_{K}^{(0)}} \sinh \left(\sqrt{\Omega_{K}^{(0)}} \chi\right)$ can be understood as $f_{K}(\chi)=\sin \chi$ (for $K=+1), f_{K}(\chi)=\chi$ (for $K=0$ ), and $f_{K}(\chi)=\sinh \chi$ (for $\left.K=-1\right)$. For the flat case $(K=0)$, Eq. (9) reduces to $d_{L}(z)=(1+z) \int_{0}^{z} \mathrm{~d} \tilde{z} / H(\tilde{z})$, i.e.

$$
H(z)=\left[\frac{\mathrm{d}}{\mathrm{d} z}\left(\frac{d_{L}(z)}{1+z}\right)\right]^{-1}
$$

Hence the measurement of the luminosity distance $d_{L}(z)$ of SN Ia allows us to find the expansion history of the Universe for $z<\mathcal{O}(1)$.

The luminosity distance $d_{L}$ is expressed in terms of an apparent magnitude $m$ and an absolute magnitude $M$ of an object, as

$$
m-M=5 \log _{10}\left(\frac{d_{L}}{10 \mathrm{pc}}\right) .
$$

The absolute magnitude $M$ at the peak of brightness is the same for any SN Ia under the assumption of standard candles, which is around $M \simeq-19[1,2]$. The luminosity distance $d_{L}(z)$ is known from Eq. (11) by observing the apparent magnitude $m$. The redshift $z$ of an object is known by measuring the wavelength $\lambda_{0}$ of light relative to its wavelength $\lambda$ in the rest frame, i.e. $z=\lambda_{0} / \lambda-1$. The observations of many SN Ia provide the dependence of the luminosity distance $d_{L}$ in terms of $z$.

Expanding the function (9) around $z=0$, it follows that

$$
\begin{aligned}
d_{L}(z) & =\frac{1}{H_{0}}\left[z+\left\{1-\frac{E^{\prime}(0)}{2}\right\} z^{2}+\mathcal{O}\left(z^{3}\right)\right] \\
& =\frac{1}{H_{0}}\left[z+\frac{1}{4}\left(1-3 w_{\mathrm{DE}} \Omega_{\mathrm{DE}}^{(0)}+\Omega_{K}^{(0)}\right) z^{2}+\mathcal{O}\left(z^{3}\right)\right]
\end{aligned}
$$

where a prime represents a derivative with respect to $z$. Note that, in the second line, we have used Eq. (8). In the presence of dark energy $\left(w_{\mathrm{DE}}<0\right.$ and $\left.\Omega_{\mathrm{DE}}^{(0)}>0\right)$ the luminosity distance gets larger than that in the flat Universe without dark energy. For smaller (negative) $w_{\mathrm{DE}}$ and for larger $\Omega_{\mathrm{DE}}^{(0)}$ this tendency becomes more significant. The open Universe without dark energy can also give rise to a larger value of $d_{L}(z)$, but the density parameter $\Omega_{K}^{(0)}$ is constrained to be close to 0 from the WMAP data (more precisely, $-0.0175<\Omega_{K}^{(0)}<0.0085$ [21]). Hence, in the low redshift regime $(z<1)$, the luminosity distance in the open Universe is hardly different from that in the flat Universe without dark energy.

As we see from Eq. (12), the observational data in the high redshift regime $(z>0.5)$ allows us to confirm the presence of dark energy. The SN Ia data released by Riess et al. [1] and Perlmutter et al. [2] in 1998 in the redshift regime $0.2<z<0.8$ showed that the luminosity distances of observed SN Ia tend to be larger than those predicted in the flat Universe without dark energy. Assuming a flat Universe with a dark energy equation of state $w_{\mathrm{DE}}=-1$ (i.e. the cosmological constant), Perlmutter et al. 2] found that the cosmological constant is present at the $99 \%$ confidence level. According to their analysis the density parameter of non-relativistic matter today was constrained to be $\Omega_{m}^{(0)}=0.28_{-0.08}^{+0.09}$ ( $68 \%$ confidence level) in the flat universe with the cosmological constant.

Over the past decade, more SN Ia data have been collected by a number of high-redshift surveys-such as SuperNova Legacy Survey (SNLS) 13], Hubble Space Telescope (HST) [14, 15], and "Equation of State: SupErNovae trace Cosmic Expansion" (ESSENCE) 16, 17] survey. These data also confirmed that the Universe entered the epoch of cosmic acceleration after the matter-dominated epoch. If we allow the case in which dark energy is different from the cosmological constant (i.e. $w_{\mathrm{DE}} \neq-1$ ), then observational constraints on $w_{\mathrm{DE}}$ and $\Omega_{\mathrm{DE}}^{(0)}\left(\right.$ or $\Omega_{m}^{(0)}$ ) are not so stringent. In Fig. 1 we show the observational contours on $\left(w_{\mathrm{DE}}, \Omega_{m}^{(0)}\right)$ for constant $w_{\mathrm{DE}}$ obtained from the "Union08" SN Ia data by Kowalski et al. [18]. Clearly the SN Ia data alone are not yet sufficient to place tight bounds on $w_{\mathrm{DE}}$.

In the flat Universe dominated by dark energy with constant $w_{\mathrm{DE}}$, it follows from Eq. (8) that $H^{2} \simeq H_{0}^{2} \Omega_{\mathrm{DE}}^{(0)}(1+$ $z)^{3\left(1+w_{\mathrm{DE}}\right)} \propto a^{-3\left(1+w_{\mathrm{DE}}\right)}$. Integrating this equation, we find that the scale factor evolves as $a \propto t^{2 /\left(3\left(1+w_{\mathrm{DE}}\right)\right)}$ for $w_{\mathrm{DE}}>-1$ and $a \propto e^{H t}$ for $w_{\mathrm{DE}}=-1$. The cosmic acceleration occurs for $-1 \leq w_{\mathrm{DE}}<-1 / 3$. In fact, Fig. 1 ishows that $w_{\mathrm{DE}}$ is constrained to be smaller than $-1 / 3$. If $w_{\mathrm{DE}}<-1$, which is called phantoms or ghosts [68], the solution corresponding to the expanding Universe is given by $a \propto\left(t_{s}-t\right)^{2 /\left(3\left(1+w_{\mathrm{DE}}\right)\right)}$, where $t_{s}$ is a constant. In this case the Universe ends at $t=t_{s}$ with a so-called big rip singularity [69, 70] at which the curvature grows toward infinity. ${ }^{1}$ The

\footnotetext{
1 There are other classes of finite-time singularities studied in Refs. 71 79$]$. In some cases quantum effects can moderate such singularities
} 


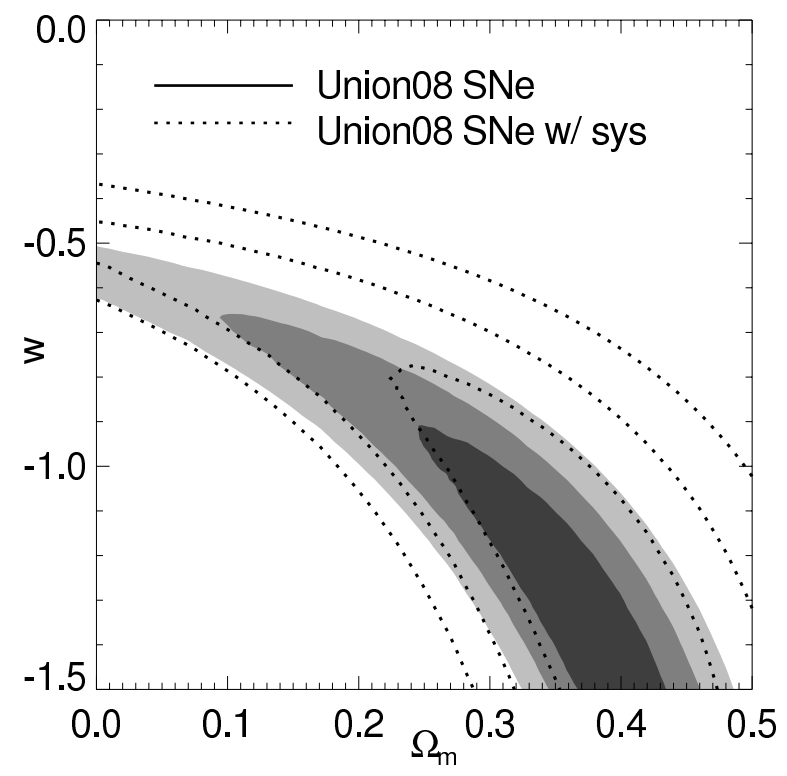

Figure 1: $68.3 \%, 95.4 \%$, and $99.7 \%$ confidence level contours on $w_{\mathrm{DE}}$ and $\Omega_{m}^{(0)}$ (denoted as $w$ and $\Omega_{m}$ in the figure) constrained by the Union08 SN Ia datasets. The equation of state $w_{\mathrm{DE}}$ is assumed to be constant. From Ref. [18].

current observations allow the possibility of the phantom equation of state. We note, however, that the dark energy equation of state smaller than -1 does not necessarily imply the appearance of the big rip singularity. In fact, in some of modified gravity models such as $f(R)$ gravity, it is possible to realize $w_{\mathrm{DE}}<-1$ without having a future big rip singularity [83].

If the dark equation of state is not constant, we need to parametrize $w_{\mathrm{DE}}$ as a function of the redshift $z$. This smoothing process is required because the actual observational data have discrete values of redshifts with systematic and statistical errors. There are several ways of parametrizations proposed so far. In general one can write such parametrizations in the form

$$
w_{\mathrm{DE}}(z)=\sum_{n=0} w_{n} x_{n}(z)
$$

where $n$ 's are integers. We show a number of examples for the expansions:

$$
\begin{array}{ll}
\text { (i) Redshift : } & x_{n}(z)=z^{n}, \\
\text { (ii) Scale factor : } & x_{n}(z)=(1-a)^{n}=\left(\frac{z}{1+z}\right)^{n}, \\
\text { (iii) Logarithmic : } & x_{n}(z)=[\ln (1+z)]^{n} .
\end{array}
$$

The parametrization (i) was introduced by Huterer and Turner [3] and Weller and Albrecht [84] with $n \leq 1$, i.e. $w_{\mathrm{DE}}=w_{0}+w_{1} z$. Chevalier and Polarski [85] and Linder [86] proposed the parametrization (ii) with $n \leq 1$, i.e.

$$
w_{\mathrm{DE}}(z)=w_{0}+w_{1}(1-a)=w_{0}+w_{1} \frac{z}{1+z} .
$$

This has a dependence $w_{\mathrm{DE}}(z)=w_{0}+w_{1}$ for $z \rightarrow \infty$ and $w_{\mathrm{DE}}(z) \rightarrow w_{0}$ for $z \rightarrow 0$. A more general form, $w_{\mathrm{DE}}(z)=w_{0}+w_{1} z /(1+z)^{p}$, was proposed by Jassal et al. [87]. The parametrization (iii) with $n \leq 1$ was introduced by Efstathiou [88]. A functional form that can be used for a fast transition of $w_{\mathrm{DE}}(z)$ was also proposed [89, 90]. In addition to the parametrization of $w_{\mathrm{DE}}$, a number of authors assumed parametric forms of $d_{L}(z)$ [91], or $H(z)$ 92 94. Many works placed observational constraints on the property of dark energy by using such parametrizations [95-109].

[75, 80-82]. 


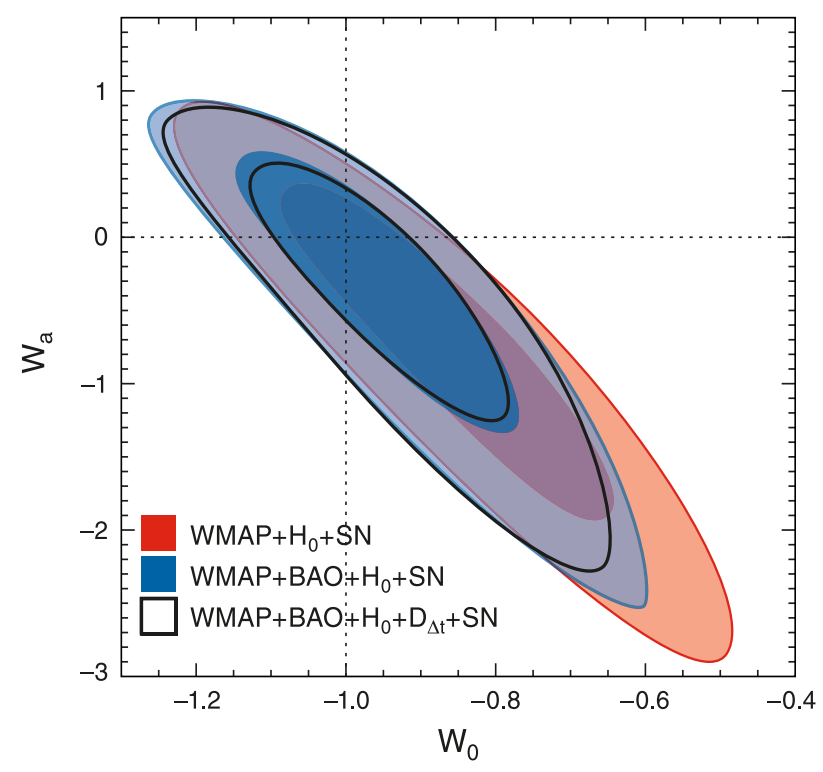

Figure 2: Observational constraints on the parameters $w_{0}$ and $w_{1}$ (denoted as $w_{a}$ in the figure) for the parametrization (17). The contours show the $68 \%$ and $95 \%$ confidence level from WMAP $+H_{0}+\mathrm{SN}$ (red), WMAP $+\mathrm{BAO}+H_{0}+\mathrm{SN}(\mathrm{blue})$, and $\mathrm{WMAP}+\mathrm{BAO}+H_{0}+D_{\Delta t}+\mathrm{SN}$ (black), for a flat universe. From Ref. 22].

In Fig. 2 we show the SN Ia constraints combined with other measurements such as the WMAP 7-year [22] and the BAO data 25]. The parametrization (17) is used in this analysis. The Gaussian prior on the present-day Hubble constant [110], $H_{0}=74.2 \pm 3.6 \mathrm{~km} \mathrm{sec}^{-1} \mathrm{Mpc}^{-1}$ (68\% confidence level), is also included in the analysis (obtained from the magnitude-redshift relation of 240 low- $z$ SN Ia at $z<0.1$ ). In Fig. 2, " $D_{\Delta t}$ " means a constraint coming from the measurement of gravitational lensing time delays [111]. The joint constraint from WMAP $+\mathrm{BAO}+H_{0}+D_{\Delta t}+\mathrm{SN}$ gives the bound

$$
w_{0}=-0.93 \pm 0.13, \quad w_{1}=-0.41_{-0.71}^{+0.72},
$$

at the $68 \%$ confidence level. Hence the current observational data are consistent with the flat Universe in the presence of the cosmological constant $\left(w_{0}=-1, w_{1}=0\right)$.

\section{B. CMB}

The temperature anisotropies in CMB are affected by the presence of dark energy. The position of the acoustic peaks in CMB anisotropies depends on the expansion history from the decoupling epoch to the present. Hence the presence of dark energy leads to the shift for the positions of acoustic peaks. There is also another effect called the Integrated-Sachs-Wolfe (ISW) effect [112] induced by the variation of the gravitational potential during the epoch of the cosmic acceleration. Since the ISW effect is limited to large-scale perturbations, the former effect is typically more important.

The cosmic inflation in the early Universe [113 116] predicts nearly scale-invariant spectra of density perturbations through the quantum fluctuation of a scalar field. This is consistent with the CMB temperature anisotropies observed by COBE [117] and WMAP [19]. The perturbations are "frozen" after the scale $\lambda=(2 \pi / k) a$ ( $k$ is a comoving wavenumber) leaves the Hubble radius $H^{-1}$ during inflation $\left(\lambda>H^{-1}\right)$ [118, 119]. After inflation, the perturbations cross inside the Hubble radius again $\left(\lambda<H^{-1}\right)$ and they start to oscillate as sound waves. This second horizon crossing occurs earlier for larger $k$ (i.e. for smaller scale perturbations).

We define the sound horizon as $r_{s}(\eta)=\int_{0}^{\eta} \mathrm{d} \tilde{\eta} c_{s}(\tilde{\eta})$, where $c_{s}$ is the sound speed and $\mathrm{d} \eta=a^{-1} \mathrm{~d} t$. The sound speed squared is given by

$$
c_{s}^{2}=1 /\left[3\left(1+R_{s}\right)\right], \quad R_{s}=3 \rho_{b} /\left(4 \rho_{\gamma}\right),
$$

where $\rho_{b}$ and $\rho_{\gamma}$ are the energy densities of baryons and photons, respectively. The characteristic angle for the location 
of CMB acoustic peaks is [120]

$$
\theta_{A} \equiv \frac{r_{s}\left(z_{\mathrm{dec}}\right)}{d_{A}^{(c)}\left(z_{\mathrm{dec}}\right)}
$$

where $d_{A}^{(c)}$ is the comoving angular diameter distance related with the luminosity distance $d_{L}$ via the duality relation $d_{A}^{(c)}=d_{L} /(1+z)$ [12, 67], and $z_{\mathrm{dec}} \simeq 1090$ is the redshift at the decoupling epoch. The CMB multipole $\ell_{A}$ that corresponds to the angle (20) is

$$
\ell_{A}=\frac{\pi}{\theta_{A}}=\pi \frac{d_{A}^{(c)}\left(z_{\mathrm{dec}}\right)}{r_{s}\left(z_{\mathrm{dec}}\right)}
$$

Using Eq. (9) and the background equation $3 H^{2}=8 \pi G\left(\rho_{m}+\rho_{r}\right)$ for the redshift $z>z_{\text {dec }}$ (where $\rho_{m}$ and $\rho_{r}$ are the energy density of non-relativistic matter and radiation, respectively), we obtain [121, 122]

$$
\ell_{A}=\frac{3 \pi}{4} \sqrt{\frac{\omega_{b}}{\omega_{\gamma}}}\left[\ln \left(\frac{\sqrt{R_{s}\left(a_{\mathrm{dec}}\right)+R_{s}\left(a_{\mathrm{eq}}\right)}+\sqrt{1+R_{s}\left(a_{\mathrm{dec}}\right)}}{1+\sqrt{R_{s}\left(a_{\mathrm{eq}}\right)}}\right)\right]^{-1} \mathcal{R}
$$

where $\omega_{b} \equiv \Omega_{b}^{(0)} h^{2}$ and $\omega_{\gamma} \equiv \Omega_{\gamma}^{(0)} h^{2}$, and $\mathcal{R}$ is the so-called CMB shift parameter defined by 123 ]

$$
\mathcal{R} \equiv \sqrt{\frac{\Omega_{m}^{(0)}}{\Omega_{K}^{(0)}}} \sinh \left(\sqrt{\Omega_{K}^{(0)}} \int_{0}^{z_{\mathrm{dec}}} \frac{\mathrm{d} z}{E(z)}\right) .
$$

The quantity $R_{s}=3 \rho_{b} /\left(4 \rho_{\gamma}\right)$ can be expressed as

$$
R_{s}(a)=\left(3 \omega_{b} / 4 \omega_{\gamma}\right) a
$$

In Eq. (22), $a_{\mathrm{dec}}$ and $a_{\mathrm{eq}}$ correspond to the scale factor at the decoupling epoch and at the radiation-matter equality, respectively.

The change of cosmic expansion history from the decoupling epoch to the present affects the CMB shift parameter, which gives rise to the shift for the multipole $\ell_{A}$. The general relation for all peaks and troughs of observed CMB anisotropies is given by [124]

$$
\ell_{m}=\ell_{A}\left(m-\phi_{m}\right),
$$

where $m$ represents peak numbers $\left(m=1\right.$ for the first peak, $m=1.5$ for the first trough,...) and $\phi_{m}$ is the shift of multipoles. For a given cosmic curvature $\Omega_{K}^{(0)}$, the quantity $\phi_{m}$ depends weakly on $\omega_{b}$ and $\omega_{m} \equiv \Omega_{m}^{(0)} h^{2}$. The shift of the first peak can be fitted as $\phi_{1}=0.265[124]$. The WMAP 5-year bound on the CMB shift parameter is given by [21]

$$
\mathcal{R}=1.710 \pm 0.019
$$

at the $68 \%$ confidence level. Taking $\mathcal{R}=1.710$ together with other values $\omega_{b}=0.02265, \omega_{m}=0.1369$, and $\omega_{\gamma}=2.469 \times 10^{-5}$ constrained by the WMAP 5-year data, we obtain $\ell_{A} \simeq 300$ from Eq. (22). Using the relation (25) with $\phi_{1}=0.265$ we find that the first acoustic peak corresponds to $\ell_{1} \simeq 220$, as observed in CMB anisotropies.

In the flat Universe $(K=0)$ the CMB shift parameter is simply given by $\mathcal{R}=\sqrt{\Omega_{m}^{(0)}} \int_{0}^{z_{\mathrm{dec}}} \mathrm{d} z / E(z)$. For smaller $\Omega_{m}^{(0)}$ (i.e. for larger $\left.\Omega_{\mathrm{DE}}^{(0)}\right), \mathcal{R}$ tends to be smaller. For the cosmological constant $\left(w_{\mathrm{DE}}=-1\right)$ the normalized Hubble expansion rate is given by $E(z)=\left[\Omega_{m}^{(0)}(1+z)^{3}+\Omega_{\mathrm{DE}}^{(0)}\right]^{1 / 2}$. Under the bound (26) the density parameter is constrained to be $0.72<\Omega_{\mathrm{DE}}^{(0)}<0.77$. This is consistent with the bound coming from the SN Ia data. One can also show that, for increasing $w_{\mathrm{DE}}$, the observationally allowed values of $\Omega_{m}^{(0)}$ gets larger. However, $\mathcal{R}$ depends weakly on the $w_{\mathrm{DE}}$. Hence the CMB data alone do not provide a tight constraint on $w_{\mathrm{DE}}$. In Fig. 3 we show the joint observational constraints on $w_{\mathrm{DE}}$ and $\Omega_{m}^{(0)}$ (for constant $w_{\mathrm{DE}}$ ) obtained from the WMAP 5-year data and the Union08 SN Ia data [18]. The joint observational constraints provide much tighter bounds compared to the individual constraint from $\mathrm{CMB}$ and SN Ia. For the flat Universe Kowalski et al. [18] obtained the bounds $w_{\mathrm{DE}}=-0.955_{-0.066-0.060}^{+0.060+0.059}$ and $\Omega_{m}^{(0)}=0.265_{-0.021}^{+0.022+0.018}$ (with statistical and systematic errors) from the combined data analysis of CMB and SN Ia. See also Refs. [95, 125 133] for related observational constraints. 

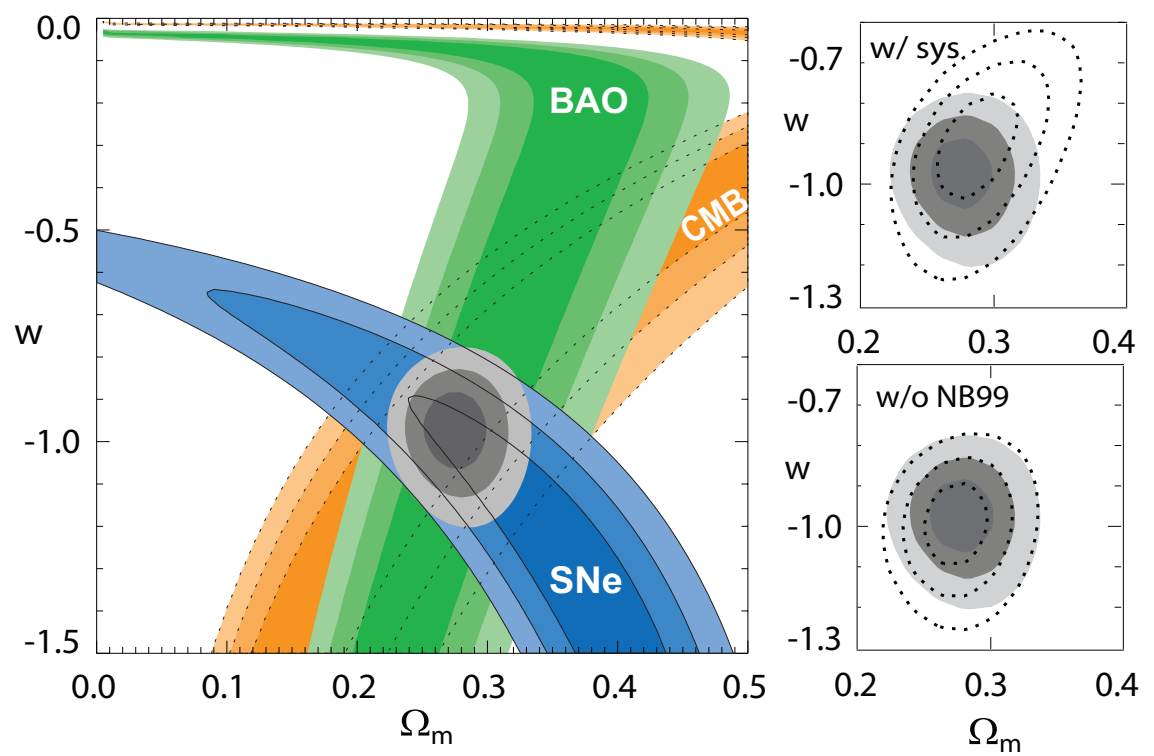

Figure 3: $68.3 \%, 95.4 \%$ and $99.7 \%$ confidence level contours on $w_{\mathrm{DE}}$ and $\Omega_{m}^{(0)}$ (denoted as $w$ and $\Omega_{m}$ in the figure, respectively) for a flat Universe. The left panel illustrates the individual constraints from SN Ia, CMB, and BAO, as well as the combined constraints (filled gray contours, statistical errors only). The upper right panel shows the effect of including systematic errors. The lower right panel illustrates the impact of the Supernova Cosmology Project (SCP) Nearby 1999 data. From Ref. [18].

\section{BAO}

The detection of baryon acoustic oscillations first reported in 2005 by Eisenstein et. al. [23] in a spectroscopic sample of 46,748 luminous red galaxies observed by the Sloan Digital Sky Survey (SDSS) has provided another test for probing the property of dark energy. Since baryons are strongly coupled to photons prior to the decoupling epoch, the oscillation of sound waves is imprinted in baryon perturbations as well as CMB anisotropies.

The sound horizon at which baryons were released from the Compton drag of photons determines the location of baryon acoustic oscillations. This epoch, called the drag epoch, occurs at the redshift $z_{d}$. The sound horizon at $z=z_{d}$ is given by $r_{s}\left(z_{d}\right)=\int_{0}^{\eta_{d}} \mathrm{~d} \eta c_{s}(\eta)$, where $c_{s}$ is the sound speed. According to the fitting formula of $z_{d}$ by Eisenstein and $\mathrm{Hu}$ [134], $z_{d}$ and $r_{s}\left(z_{d}\right)$ are constrained to be around $z_{d} \approx 1020$ and $r_{s}\left(z_{d}\right) \approx 150 \mathrm{Mpc}$.

We observe the angular and redshift distributions of galaxies as a power spectrum $P\left(k_{\perp}, k_{\|}\right)$in the redshift space, where $k_{\perp}$ and $k_{\|}$are the wavenumbers perpendicular and parallel to the direction of light respectively. In principle we can measure the following two ratios [135]

$$
\theta_{s}(z)=\frac{r_{s}\left(z_{d}\right)}{d_{A}^{(c)}(z)}, \quad \delta z_{s}(z)=\frac{r_{s}\left(z_{d}\right) H(z)}{c},
$$

where the speed of light $c$ is recovered for clarity. In the first equation $d_{A}^{(c)}$ is the comoving angular diameter distance related with the proper angular diameter distance $d_{A}$ via the relation $d_{A}^{(c)}=d_{A} / a=d_{A}(1+z)$. The quantity $\theta_{s}(z)$ characterizes the angle orthogonal to the line of sight, whereas the quantity $\delta z_{s}$ corresponds to the oscillations along the line of sight.

The current $\mathrm{BAO}$ observations are not sufficient to measure both $\theta_{s}(z)$ and $\delta z_{s}(z)$ independently. From the spherically averaged spectrum one can find a combined distance scale ratio given by [135]

$$
\left[\theta_{s}(z)^{2} \delta z_{s}(z)\right]^{1 / 3} \equiv \frac{r_{s}\left(z_{d}\right)}{\left[(1+z)^{2} d_{A}^{2}(z) c / H(z)\right]^{1 / 3}}
$$

or, alternatively, the effective distance ratio [23]

$$
D_{V}(z) \equiv\left[(1+z)^{2} d_{A}^{2}(z) c z / H(z)\right]^{1 / 3} .
$$

In 2005 Eisenstein et al. [23] obtained the constraint $D_{V}(z)=1370 \pm 64 \mathrm{Mpc}$ at the redshift $z=0.35$. In 2007 Percival et al. 24] measured the effective distance ratio defined by

$$
r_{\mathrm{BAO}}(z) \equiv r_{s}\left(z_{d}\right) / D_{V}(z),
$$


at the two redshifts: $r_{\mathrm{BAO}}(z=0.2)=0.1980 \pm 0.0058$ and $r_{\mathrm{BAO}}(z=0.35)=0.1094 \pm 0.0033$. This is based on the data from the 2-degree Field (2dF) Galaxy Redshift Survey. These data provide the observational contour of BAO plotted in Fig. 3. From the joint data analysis of SN Ia [18], WMAP 5-year [21], and BAO data [24], Kowalski et al. [18] placed the constraints $w_{\mathrm{DE}}=-0.969_{-0.063}^{+0.059}(\mathrm{stat})_{-0.066}^{+0.063}$ (sys) and $\Omega_{m}^{(0)}=0.274_{-0.016}^{+0.016}(\mathrm{stat})_{-0.012}^{+0.013}$ (sys) for the constant equation of state of dark energy.

The recent measurement of the $2 \mathrm{dF}$ as well as the SDSS data provided the effective distance ratio to be $r_{\mathrm{BAO}}(z=$ $0.2)=0.1905 \pm 0.0061$ and $r_{\mathrm{BAO}}(z=0.35)=0.1097 \pm 0.0036[25]$. Using these data together with the WMAP 7-year data 22] and the Gaussian prior on the Hubble constant $H_{0}=74.2 \pm 3.6 \mathrm{~km} \mathrm{sec}^{-1} \mathrm{Mpc}^{-1}$ [110], Komatsu et al. 22] derived the constraint $w_{\mathrm{DE}}=-1.10 \pm 0.14$ (68\% confidence level) for the constant equation of state in the flat Universe. Adding the high- $z$ SN Ia in their analysis they found the most stringent bound: $w_{\mathrm{DE}}=-0.980 \pm 0.053$ (68 $\%$ confidence level). Hence the $\Lambda \mathrm{CDM}$ model is well consistent with a number of independent observational data.

Finally we should mention that there are other constraints coming from the cosmic age [136], large-scale clustering [137 139], gamma ray bursts [140 144], and weak lensing [145-150]. So far we have not found strong evidence for supporting dynamical dark energy models over the $\Lambda$ CDM model, but future high-precision observations may break this degeneracy.

\section{COSMOLOGICAL CONSTANT}

The cosmological constant $\Lambda$ is one of the simplest candidates of dark energy, and as we have seen in the previous section, it is favored by a number of observations. However, if the origin of the cosmological constant is a vacuum energy, it suffers from a serious problem of its energy scale relative to the dark energy density today [26]. The zero-point energy of some field of mass $m$ with momentum $k$ and frequency $\omega$ is given by $E=\omega / 2=\sqrt{k^{2}+m^{2}} / 2$. Summing over the zero-point energies of this field up to a cut-off scale $k_{\max }(\gg m)$, we obtain the vacuum energy density

$$
\rho_{\mathrm{vac}}=\int_{0}^{k_{\max }} \frac{\mathrm{d}^{3} k}{(2 \pi)^{3}} \frac{1}{2} \sqrt{k^{2}+m^{2}} .
$$

Since the integral is dominated by the mode with large $k(\gg m)$, we find that

$$
\rho_{\mathrm{vac}} \approx \int_{0}^{k_{\max }} \frac{4 \pi k^{2} \mathrm{~d} k}{(2 \pi)^{3}} \frac{1}{2} k=\frac{k_{\max }^{4}}{16 \pi^{2}} .
$$

Taking the cut-off scale $k_{\max }$ to be the Planck mass $m_{\mathrm{pl}}$, the vacuum energy density can be estimated as $\rho_{\mathrm{vac}} \simeq$ $10^{74} \mathrm{GeV}^{4}$. This is about $10^{121}$ times larger than the observed value $\rho_{\mathrm{DE}}^{(0)} \simeq 10^{-47} \mathrm{GeV}^{4}$.

Before the observational discovery of dark energy in 1998, most people believed that the cosmological constant is exactly zero and tried to explain why it is so. The vanishing of a constant may imply the existence of some symmetry. In supersymmetric theories the bosonic degree of freedom has its Fermi counter part which contributes to the zero point energy with an opposite sign ${ }^{2}$. If supersymmetry is unbroken, an equal number of bosonic and fermionic degrees of freedom is present such that the total vacuum energy vanishes. However it is known that supersymmetry is broken at sufficient high energies (for the typical scale $M_{\text {SUSY }} \approx 10^{3} \mathrm{GeV}$ ). Therefore the vacuum energy is generally non-zero in the world of broken supersymmetry.

Even if supersymmetry is broken there is a hope to obtain a vanishing $\Lambda$ or a tiny amount of $\Lambda$. In supergravity theory the effective cosmological constant is given by an expectation value of the potential $V$ for chiral scalar fields $\varphi^{i}$ [151]:

$$
V\left(\varphi, \varphi^{*}\right)=e^{\kappa^{2} K}\left[D_{i} W\left(K^{i j^{*}}\right)\left(D_{j} W\right)^{*}-3 \kappa^{2}|W|^{2}\right],
$$

where $K$ and $W$ are the so-called Kähler potential and the superpotential, respectively, which are the functions of $\varphi^{i}$ and its complex conjugate $\varphi^{i *}$. The quantity $K^{i j^{*}}$ is an inverse of the derivative $K_{i j^{*}} \equiv \partial^{2} K / \partial \varphi^{i} \partial \varphi^{j^{*}}$, whereas the derivative $D_{i} W$ is defined by $D_{i} W \equiv \partial W / \partial \varphi^{i}+\kappa^{2} W\left(\partial K / \partial \varphi^{i}\right)$.

The condition $D_{i} W \neq 0$ corresponds to the breaking of supersymmetry. In this case it is possible to find scalar field values leading to the vanishing potential $(V=0)$, but this is not in general an equilibrium point of the potential

\footnotetext{
2 The readers who are not familiar with supersymmetric theories may consult the books [151, 152].
} 
$V$. Nevertheless there is a class of Kähler potentials and superpotentials giving a stationary scalar-field configuration at $V=0$. The gluino condensation model in $E_{8} \times E_{8}$ superstring theory proposed by Dine [153] belongs to this class. The reduction of the 10-dimensional action to the 4-dimensional action gives rise to a so-called modulus field $T$. This field characterizes the scale of the compactified 6-dimensional manifold. Generally one has another complex scalar field $S$ corresponding to 4-dimensional dilaton/axion fields. The fields $T$ and $S$ are governed by the Kähler potential

$$
K(T, S)=-\left(3 / \kappa^{2}\right) \ln \left(T+T^{*}\right)-\left(1 / \kappa^{2}\right) \ln \left(S+S^{*}\right),
$$

where $\left(T+T^{*}\right)$ and $\left(S+S^{*}\right)$ are positive definite. The field $S$ couples to the gauge fields, while $T$ does not. An effective superpotential for $S$ can be obtained by integrating out the gauge fields under the use of the $R$-invariance [154]:

$$
W(S)=M_{\mathrm{pl}}^{3}\left[c_{1}+c_{2} \exp \left(-3 S / 2 c_{3}\right)\right]
$$

where $c_{1}, c_{2}$, and $c_{3}$ are constants.

Substituting Eqs. (34) and (35) into Eqs. (33), we obtain the field potential

$$
\begin{aligned}
V & =\frac{1}{\left(T+T^{*}\right)^{3}\left(S+S^{*}\right)}\left(D_{S} W\right) K^{S S^{*}}\left(D_{S} W\right)^{*} \\
& =\frac{M_{\mathrm{pl}}^{4}}{\left(T+T^{*}\right)^{3}\left(S+S^{*}\right)}\left|c_{1}+c_{2} \exp \left(-3 S / 2 c_{3}\right)\left\{1+\frac{3}{2 c_{3}}\left(S+S^{*}\right)\right\}\right|^{2},
\end{aligned}
$$

where, in the first line, we have used the property $\left(D_{T} W\right) K^{T T^{*}}\left(D_{T} W\right)^{*}=3 \kappa^{2}|W|^{2}$ for the modulus term. This potential is positive because of the cancellation of the last term in Eq. (33). The stationary field configuration with $V=0$ is realized under the condition $D_{S} W=\partial W / \partial S-W /\left(S+S^{*}\right)=0$. The derivative, $D_{T} W=\kappa^{2} W \partial K / \partial T=$ $-3 W /\left(T+T^{*}\right)$, does not necessarily vanish. When $D_{T} W \neq 0$ the supersymmetry is broken with a vanishing potential energy. Therefore it is possible to obtain a stationary field configuration with $V=0$ even if supersymmetry is broken.

The discussion above is based on the lowest-order perturbation theory. This picture is not necessarily valid to all finite orders of perturbation theory because the non-supersymmetric field configuration is not protected by any symmetry. Moreover some non-perturbative effect can provide a large contribution to the effective cosmological constant [43]. The so-called flux compactification in type IIB string theory allows us to realize a metastable de Sitter (dS) vacuum by taking into account a non-perturbative correction to the superpotential (coming from brane instantons) as well as a number of anti D3-branes in a warped geometry [27]. Hence it is not hopeless to obtain a small value of $\Lambda$ or a vanishing $\Lambda$ even in the presence of some non-perturbative corrections.

Kachru, Kallosh, Linde and Trivedi (KKLT) 27] constructed dS solutions in type II string theory compactified on a Calabi-Yau manifold in the presence of flux. The construction of the dS vacua in the KKLT scenario consists of two steps. The first step is to freeze all moduli fields in the flux compactification at a supersymmetric Anti de Sitter (AdS) vacuum. Then a small number of the anti D3-brane is added in a warped geometry with a throat, so that the AdS minimum is uplifted to yield a dS vacuum with broken supersymmetry. If we want to use the KKLT dS minimum derived above for the present cosmic acceleration, we require that the potential energy $V_{\mathrm{dS}}$ at the minimum is of the order of $V_{\mathrm{dS}} \simeq 10^{-47} \mathrm{GeV}^{4}$. Depending on the number of fluxes there are a vast of dS vacua, which opened up a notion called string landscape [155].

The question why the vacuum we live in has a very small energy density among many possible vacua has been sometimes answered with the anthropic principle [156, 157]. Using the anthropic arguments, Weinberg put the bound on the vacuum energy density [158]

$$
-10^{-123} m_{\mathrm{pl}}^{4} \lesssim \rho_{\Lambda} \lesssim 3 \times 10^{-121} m_{\mathrm{pl}}^{4} .
$$

The upper bound comes from the requirement that the vacuum energy does not dominate over the matter density for the redshift $z \gtrsim 1$. Meanwhile the lower bound comes from the condition that $\rho_{\Lambda}$ does not cancel the present cosmological density. Some people have studied landscape statistics by considering the relative abundance of longlived low-energy vacua satisfying the bound (37) [159 162]. These statistical approaches are still under study, but it will be interesting to pursue the possibility to obtain high probabilities for the appearance of low-energy vacua.

Even in 1980s there were some pioneering works for finding a mechanism to make the effective cosmological constant small. For example, let us consider a 4 -form field $F^{\mu \nu \lambda \sigma}$ expressed by a unit totally anti-symmetric tensor $\epsilon^{\mu \nu \lambda \sigma}$, as $F^{\mu \nu \lambda \sigma}=c \epsilon^{\mu \nu \lambda \sigma}$ ( $c$ is a constant). Then the energy density of the 4-form field is given by $F^{\mu \nu \lambda \sigma} F_{\mu \nu \lambda \sigma} /(2 \cdot 4 !)=c^{2} / 2$. Taking into account a scalar field $\phi$ with a potential energy $V(\phi)$, the total energy density is $\Lambda=V(\phi)+c^{2} / 2$. In 1984 Linde 163] considered the quantum creation of the Universe and claimed that the final value of $\Lambda$ can appear with approximately the same probability because $V(\phi)$ can take any initial value such that $\Lambda \approx m_{\mathrm{pl}}^{4}$. 
In 1987-1988 Brown and Teltelboim [164, 165] studied the quantum creation of closed membranes by totally antisymmetric tensor and gravitational fields to neutralize the effective cosmological constant with small values. The constant $c$ appearing in the energy density of the 4-form field can be quantized in integer multiples of the membrane charge $q$, i.e. $c=n q$. If we consider a negative bare cosmological constant $-\Lambda_{b}$ (as in the KKLT model) in the presence of the flux energy density $n^{2} q^{2} / 2$, then the effective gravitational constant is given by $\Lambda=-\Lambda_{b}+n^{2} q^{2} / 2$. The field strength of the 4 -form field is slowly discharged by a quantum Schwinger pair creation of field sources $[n q \rightarrow(n-1) q]$. However, in order to get a tiny value of $\Lambda$ consistent with the dark energy density today, the membrane change $q$ is constrained to be very small (for natural choices of $\Lambda_{b}$ ) [166].

In 2000 Bousso and Polchinski [167] considered multiple 4-form fields that arise in M-theory compactifications and showed that the small value of $\Lambda$ can be explained for natural choices of $q$. More precisely, if we consider $J(>1)$ 4 -form fields as well as $J$ membrane species with charges $q_{1}, q_{2}, \cdots, q_{J}$ and the quantized flux $F_{i}^{\mu \nu \lambda \sigma}=n_{i} q_{i} \epsilon^{\mu \nu \lambda \sigma}$, the effective cosmological constant is given by

$$
\Lambda=-\Lambda_{b}+\sum_{i=1}^{J} n_{i}^{2} q_{i}^{2} / 2
$$

Bousso and Polchinski [167] showed that, for natural values of charges $\left(q_{i}<\mathcal{O}(0.1)\right)$ there exists integers $n_{i}$ such that $2 \Lambda_{b}<\sum_{i=1}^{J} n_{i}^{2} q_{i}^{2}<2\left(\Lambda_{b}+\Delta \Lambda\right)$ with $\Delta \Lambda \approx 10^{-47} \mathrm{GeV}^{4}$. This can be realized for $J>100$ and $\Lambda_{b} \approx m_{\mathrm{pl}}^{4}$.

There are some interesting works for decoupling $\Lambda$ from gravity. In the cascading gravity scenario proposed in Ref. [168] the cosmological constant can be made gravitationally inactive by shutting off large-scale density perturbations. In Ref. [169] an incompressible gravitational Aether fluid was introduced to degraviate the vacuum. In Refs. [170, 171] Padmanabhan showed an example to gauge away the cosmological constant from gravity according to the variational principle different from the standard method. See also Refs. [172 184] for other possibilities to solve the cosmological constant problem. If the cosmological constant is completely decoupled from gravity, it is required to find alternative models of dark energy consistent with observations.

In the subsequent sections we shall consider alternative models of dark energy, under the assumption that the cosmological constant problem is solved in such a way that it vanishes completely.

\section{MODIFIED MATTER MODELS}

In this section we discuss "modified matter models" in which the energy-momentum tensor $T_{\mu \nu}$ on the r.h.s. of the Einstein equations contains an exotic matter source with a negative pressure. The models that belong to this class are quintessence, k-essence, coupled dark energy, and generalized Chaplygin gas.

\section{A. Quintessence}

A canonical scalar field $\phi$ responsible for dark energy is dubbed quintessence [36, 37] (see also Refs. 28 - 34] for earlier works). The action of quintessence is described by

$$
S=\int \mathrm{d}^{4} x \sqrt{-g}\left[\frac{1}{2 \kappa^{2}} R-\frac{1}{2} g^{\mu \nu} \partial_{\mu} \phi \partial_{\nu} \phi-V(\phi)\right]+S_{M},
$$

where $R$ is a Ricci scalar, and $\phi$ is a scalar field with a potential $V(\phi)$. As a matter action $S_{M}$, we consider perfect fluids of radiation (energy density $\rho_{r}$, equation of state $w_{r}=1 / 3$ ) and non-relativistic matter (energy density $\rho_{m}$, equation of state $\left.w_{m}=0\right)$.

In the flat FLRW background radiation and non-relativistic matter satisfy the continuity equations $\dot{\rho}_{r}+4 H \rho_{r}=0$ and $\dot{\rho}_{m}+3 H \rho_{m}=0$, respectively. The energy density $\rho_{\phi}$ and the pressure $P_{\phi}$ of the field are $\rho_{\phi}=\dot{\phi}^{2} / 2+V(\phi)$ and $P_{\phi}=\dot{\phi}^{2} / 2-V(\phi)$, respectively. The continuity equation, $\dot{\rho}_{\phi}+3 H\left(\rho_{\phi}+P_{\phi}\right)=0$, translates to

$$
\ddot{\phi}+3 H \dot{\phi}+V_{, \phi}=0
$$

where $V_{, \phi} \equiv \mathrm{d} V / \mathrm{d} \phi$. The field equation of state is given by

$$
w_{\phi} \equiv \frac{P_{\phi}}{\rho_{\phi}}=\frac{\dot{\phi}^{2}-2 V(\phi)}{\dot{\phi}^{2}+2 V(\phi)} .
$$


From the Einstein equations (4) we obtain the following equations

$$
\begin{aligned}
H^{2} & =\frac{\kappa^{2}}{3}\left[\frac{1}{2} \dot{\phi}^{2}+V(\phi)+\rho_{m}+\rho_{r}\right], \\
\dot{H} & =-\frac{\kappa^{2}}{2}\left(\dot{\phi}^{2}+\rho_{m}+\frac{4}{3} \rho_{r}\right) .
\end{aligned}
$$

Although $\left\{\rho_{r}, \rho_{m}\right\} \gg \rho_{\phi}$ during radiation and matter eras, the field energy density needs to dominate at late times to be responsible for dark energy. The condition to realize the late-time cosmic acceleration corresponds to $w_{\phi}<-1 / 3$, i.e. $\dot{\phi}^{2}<V(\phi)$ from Eq. (41). This means that the scalar potential needs to be flat enough for the field to evolve slowly. If the dominant contribution to the energy density of the Universe is the slowly rolling scalar field satisfying the condition $\dot{\phi}^{2} \ll V(\phi)$, we obtain the approximate relations $3 H \dot{\phi}+V_{, \phi} \simeq 0$ and $3 H^{2} \simeq \kappa^{2} V(\phi)$ from Eqs. (40) and (42), respectively. Hence the field equation of state in Eq. (41) is approximately given by

$$
w_{\phi} \simeq-1+2 \epsilon_{s} / 3
$$

where $\epsilon_{s} \equiv\left(V_{, \phi} / V\right)^{2} /\left(2 \kappa^{2}\right)$ is the so-called slow-roll parameter [118]. During the accelerated expansion of the Universe, $\epsilon_{s}$ is much smaller than 1 because the potential is sufficiently flat. Unlike the cosmological constant, the field equation of state deviates from $-1\left(w_{\phi}>-1\right)$.

Introducing the dimensionless variables $x_{1} \equiv \kappa \dot{\phi} /(\sqrt{6} H), x_{2} \equiv \kappa \sqrt{V} /(\sqrt{3} H)$, and $x_{3} \equiv \kappa \sqrt{\rho_{r}} /(\sqrt{3} H)$, we obtain the following equations from Eqs. (40), (42), and (43) [9, 35, 185, 186]:

$$
\begin{aligned}
& x_{1}^{\prime}=-3 x_{1}+\frac{\sqrt{6}}{2} \lambda x_{2}^{2}+\frac{1}{2} x_{1}\left(3+3 x_{1}^{2}-3 x_{2}^{2}+x_{3}^{2}\right), \\
& x_{2}^{\prime}=-\frac{\sqrt{6}}{2} \lambda x_{1} x_{2}+\frac{1}{2} x_{2}\left(3+3 x_{1}^{2}-3 x_{2}^{2}+x_{3}^{2}\right), \\
& x_{3}^{\prime}=-2 x_{3}+\frac{1}{2} x_{3}\left(3+3 x_{1}^{2}-3 x_{2}^{2}+x_{3}^{2}\right),
\end{aligned}
$$

where a prime represents a derivative with respect to $N=\ln a$, and $\lambda$ is defined by $\lambda \equiv-V_{, \phi} /(\kappa V)$. The density parameters of the field, radiation, and non-relativistic matter are given by $\Omega_{\phi}=x_{1}^{2}+x_{2}^{2}, \Omega_{r}=x_{3}^{2}$, and $\Omega_{m}=$ $1-x_{1}^{2}-x_{2}^{2}-x_{3}^{2}$, respectively. One has constant $\lambda$ for the exponential potential [35]

$$
V(\phi)=V_{0} e^{-\kappa \lambda \phi}
$$

in which case the fixed points of the system (45)-(47) can be derived by setting $x_{i}^{\prime}=0(i=1,2,3)$. The fixed point that can be used for dark energy is given by

$$
\left(x_{1}, x_{2}, x_{3}\right)=\left(\lambda / \sqrt{6}, \sqrt{1-\lambda^{2} / 6}, 0\right), \quad w_{\phi}=-1+\lambda^{2} / 3, \quad \Omega_{\phi}=1 .
$$

The cosmic acceleration can be realized for $w_{\phi}<-1 / 3$, i.e. $\lambda^{2}<2$. One can show that in this case the accelerated fixed point is a stable attractor [35]. Hence the solutions finally approach the fixed point (49) after the matter era [characterized by the fixed point $\left.\left(x_{1}, x_{2}, x_{3}\right)=(0,0,0)\right]$.

If $\lambda$ varies with time, we have the following relation

$$
\lambda^{\prime}=-\sqrt{6} \lambda^{2}(\Gamma-1) x_{1}
$$

where $\Gamma \equiv V V_{, \phi \phi} / V_{, \phi}^{2}$. For monotonically decreasing potentials one has $\lambda>0$ and $x_{1}>0$ for $V_{, \phi}<0$ and $\lambda<0$ and $x_{1}<0$ for $V_{, \phi}>0$. If the condition

$$
\Gamma=\frac{V V_{, \phi \phi}}{V_{, \phi}^{2}}>1,
$$

is satisfied, the absolute value of $\lambda$ decreases toward 0 irrespective of the signs of $V_{, \phi}[38$ ]. Then the solutions finally approach the accelerated "instantaneous" fixed point (49) even if $\lambda^{2}$ is larger than 2 during radiation and matter eras [185, 186]. In this case the field equation of state gradually decreases to -1 , so the models showing this behavior are called "freezing" models [187]. The condition (51) is the so-called tracking condition under which the field density eventually catches up that of the background fluid. 


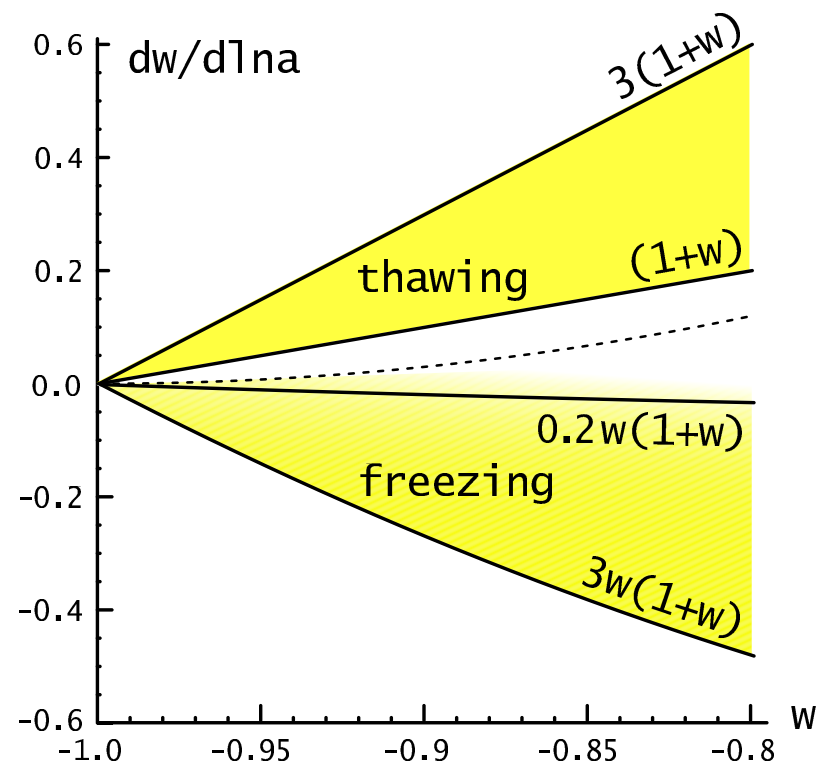

Figure 4: The allowed region in the $\left(w_{\phi}, w_{\phi}^{\prime}\right)$ plane for thawing and freezing models of quintessence $\left(w_{\phi}\right.$ is denoted as $w$ in the figure). The thawing models correspond to the region between two curves: (a) $w_{\phi}^{\prime}=3\left(1+w_{\phi}\right)$ and (b) $w_{\phi}^{\prime}=1+w_{\phi}$, whereas the freezing models are characterized by the region between two curves: (c) $w_{\phi}^{\prime}=0.2 w_{\phi}\left(1+w_{\phi}\right)$ and $(\mathrm{d}) w_{\phi}^{\prime}=3 w_{\phi}\left(1+w_{\phi}\right)$. The dotted line shows the border between the acceleration and deceleration of the field $(\ddot{\phi}=0)$, which corresponds to $w_{\phi}^{\prime}=3\left(1+w_{\phi}\right)^{2}$. From Ref. [187].

A representative potential of the freezing model is the inverse power-law potential $V(\phi)=M^{4+n} \phi^{-n}(n>0)$ [31, 38], which can appear in the fermion condensate model as a dynamical supersymmetry breaking [188]. In this case one has $\Gamma=(n+1) / n>1$ and hence the tracking condition is satisfied. Unlike the cosmological constant, even if the field energy density is not negligible relative to the background fluid density around the beginning of the radiation era, the field eventually enters the tracking regime to lead to the late-time cosmic acceleration [38]. Another example of freezing models is $V(\phi)=M^{4+n} \phi^{-n} \exp \left(\alpha \phi^{2} / m_{\mathrm{pl}}^{2}\right)$, which has a minimum with a positive energy density at which the field is eventually trapped. This potential is motivated in the framework of supergravity [189].

There is another class of quintessence potentials called "thawing" models [187]. In thawing models the field with mass $m_{\phi}$ has been frozen by the Hubble friction (i.e. the term $H \dot{\phi}$ ) until recently and then it begins to evolve after $H$ drops below $m_{\phi}$. At early times the equation of state of dark energy is $w_{\phi} \simeq-1$, but it begins to grow for $H<m_{\phi}$. The representative potentials that belong to this class are (a) $V(\phi)=V_{0}+M^{4-n} \phi^{n}(n>0)$ and (b) $V(\phi)=M^{4} \cos ^{2}(\phi / f)$. The potential (a) with $n=1$ was originally proposed by Linde [190] to replace the cosmological constant by a slowly evolving scalar field. In Ref. 191] this was revised to allow for negative values of $V(\phi)$. The universe will collapse in the future if the system enters the region with $V(\phi)<0$. The potential (b) is motivated by the Pseudo-Nambu-Goldstone Boson (PNGB), which was introduced in Ref. [192] in response to the first tentative suggestions for the existence of the cosmological constant. The small mass of the PNGB model required for dark energy is protected against radiative corrections, so this model is favored theoretically. In fact there are a number of interesting works to explain the small energy scale $M \approx 10^{-3} \mathrm{eV}$ required for the PNGB quintessence in supersymmetric theories [193 196]. See Refs. 197 202, 204 208] for the construction of quintessence potentials in the framework of supersymmetric theories.

The freezing models and the thawing models are characterized by the conditions $w_{\phi}^{\prime} \equiv \mathrm{d} w_{\phi} / \mathrm{d} N<0$ and $w_{\phi}^{\prime}>0$, respectively. More precisely the allowed regions for the freezing and thawing models are given by $3 w_{\phi}\left(1+w_{\phi}\right) \lesssim w_{\phi}^{\prime} \lesssim 0.2 w_{\phi}\left(1+w_{\phi}\right)$ and $1+w_{\phi} \lesssim w_{\phi}^{\prime} \lesssim 3\left(1+w_{\phi}\right)$, respectively [187] (see Ref. [209] for details). These

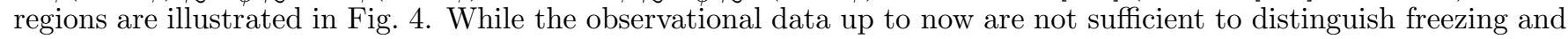
thawing models by the variation of $w_{\phi}$, we may be able to do so with the next decade high-precision observations.

There is also another useful measure called "statefinder" [210] by which the quintessence models can be distinguished from the $\Lambda$ CDM model (see also Ref. [211]). The statefinder parameters are defined by

$$
r \equiv \frac{1}{a H^{3}} \frac{\mathrm{d}^{3} a}{\mathrm{~d} t^{3}}, \quad s \equiv \frac{r-1}{3(q-1 / 2)},
$$

where $q=-\ddot{a} /\left(a H^{2}\right)$ is the deceleration parameter. Let us consider the case in which the dark energy density $\rho_{\mathrm{DE}}$ 
satisfies the continuity equation $\dot{\rho}_{\mathrm{DE}}+3 H\left(1+w_{\mathrm{DE}}\right) \rho_{\mathrm{DE}}=0$. In Einstein gravity in which the Friedmann equation $3 H^{2}=\kappa^{2}\left(\rho_{\mathrm{DE}}+\rho_{m}\right)$ holds, we obtain

$$
r=1+\frac{9 w_{\mathrm{DE}} \Omega_{\mathrm{DE}}}{2} s, \quad s=1+w_{\mathrm{DE}}-\frac{\dot{w}_{\mathrm{DE}}}{3 H w_{\mathrm{DE}}},
$$

where $\Omega_{\mathrm{DE}} \equiv \kappa^{2} \rho_{\mathrm{DE}} /\left(3 H^{2}\right)$. The $\Lambda \mathrm{CDM}$ model $\left(w_{\mathrm{DE}}=-1\right)$ corresponds to a point $(r, s)=(1,0)$ in the $(r, s)$ plane, but the quintessence models are characterized by the curves in the region $r<1$ and $s>0$ [93]. The Chaplygin gas model we discuss in Sec. IVD gives rise to the curves in the region $r>1$ and $s<0$. Hence one can distinguish between dark energy models by using the statefinders defined in Eq. (52).

It is possible to reconstruct quintessence potentials from the observational data of SN Ia. Neglecting the contribution of radiation and using the relation $\rho_{m}=\rho_{m}^{(0)}(1+z)^{3}$ for non-relativistic matter, we get the following equations from Eqs. (42) and (43) [91, 92, 212, 213]:

$$
\begin{aligned}
& \frac{\kappa^{2}}{2}\left(\frac{\mathrm{d} \phi}{\mathrm{d} z}\right)^{2}=\frac{1}{1+z} \frac{\mathrm{d} \ln E(z)}{\mathrm{d} z}-\frac{3 \Omega_{m}^{(0)}}{2} \frac{1+z}{E(z)^{2}}, \\
& \frac{\kappa^{2} V}{3 H_{0}^{2}}=E(z)-\frac{1+z}{6} \frac{\mathrm{d} E(z)^{2}}{\mathrm{~d} z}-\frac{1}{2} \Omega_{m}^{(0)}(1+z)^{3},
\end{aligned}
$$

where $E(z)=H(z) / H_{0}$. Note that we have changed the time derivative to the derivative with respect to the redshift $z$, by using the relation $\mathrm{d} t=-\mathrm{d} z /[H(1+z)]$. Integrating Eq. (54), the field $\phi$ is known as a function of $z$. Inverting $\phi(z)$ to $z(\phi)$ and plugging it into Eq. (55), one can reconstruct the potential $V$ with respect to $\phi$ by using the information of the observationally known values of $H(z)$ and $H^{\prime}(z)$ as well as $\Omega_{m}^{(0)}$. We caution, however, that the actual observational data (such as the luminosity distance) are obtained at discrete values of redshifts. Hence we need some smoothing process for reconstructing the potential $V(\phi)$ and the field equation of state $w_{\phi}(z)$. This smoothing was already discussed in Sec. II A.

From the viewpoint of particle physics, the quintessence energy density can be comparable to the background fluid density in the early Universe. It is possible to construct quintessence models in which the field energy density is proportional to the fluid density during radiation and matter eras. For the exponential potential (48) there is a fixed point giving a constant field density parameter $\Omega_{\phi}=3\left(1+w_{M}\right) / \lambda^{2}$ with $w_{\phi}=w_{M}$, where $w_{M}$ is the fluid equation of state [35]. This is called a scaling solution, which is stable for $\lambda^{2}>3\left(1+w_{M}\right)$. During radiation and matter eras one has $\Omega_{\phi}=4 / \lambda^{2}$ and $\Omega_{\phi}=3 / \lambda^{2}$, respectively. The Big Bang Nucleosynthesis (BBN) places the bound $\Omega_{\phi}<0.045(95$ $\%$ confidence level) around the temperature $T=1 \mathrm{MeV}$ [214], which gives the bound $\lambda>9.4$. In this case, however, the scalar field does not exit to the accelerated fixed point given by Eq. (49).

There are several ways to allow a transition from the scaling regime to the epoch of cosmic acceleration. One of them is to introduce a field potential that becomes shallow at late times, $V(\phi)=c_{1} e^{-\kappa \lambda \phi}+c_{2} e^{-\kappa \mu \phi}$ with $\lambda^{2}>3\left(1+w_{M}\right)$ and $\mu^{2}<2$ [215] (see Refs. 214, 216 221] for related works). For this double exponential potential the field equation of state of the final attractor is given by $w_{\phi}=-1+\mu^{2} / 3$. Another way is to consider multiple scalar fields with exponential potentials, $V\left(\phi_{1}, \phi_{2}\right)=c_{1} e^{-\kappa \lambda_{1} \phi_{1}}+c_{2} e^{-\kappa \lambda_{2} \phi_{2}}$ [222, 223]. In this case the phenomenon called assisted inflation 224] occurs for the multi-field exponential potential, even if the individual field has too steep a potential to lead to cosmic acceleration. The scalar field equation of state finally approaches the value $w_{\phi}=-1+\lambda_{\text {eff }}^{2} / 3$, where $\lambda_{\text {eff }}=\left(1 / \lambda_{1}^{2}+1 / \lambda_{2}^{2}\right)^{-1 / 2}$ is smaller than each $\lambda_{i}(i=1,2)$. In the presence of three assisting scalar fields it is possible to realize the observational bound $w_{\phi}(z=0)<-0.8$ today [225].

There is a class of models dubbed quintessential inflation [226] in which a single scalar field $\phi$ is responsible for both inflation and dark energy. One example of quintessential inflation is given by $V(\phi)=\lambda\left(\phi^{4}+M^{4}\right)$ for $\phi<0$ and $V(\phi)=\lambda M^{4} /\left[1+(\phi / M)^{n}\right](n>0)$ for $\phi \geq 0$. In the regime $\phi<0$ with $|\phi| \gg M$ the potential behaves as $V(\phi) \simeq \lambda \phi^{4}$, which leads to inflation. In the regime $\phi>0$ with $\phi \gg M$ one has $V(\phi) \simeq \lambda M^{4+n} \phi^{-n}$, which leads the late-time cosmic acceleration. Since the potential does not have a minimum, the reheating after inflation proceeds with a gravitational particle production. Although this process is not efficient in general, it may be possible to make the reheating more efficient under the instant preheating mechanism proposed in Ref. [227]. See Refs. [228 [239] about related works on quintessential inflation.

\section{B. k-essence}

Scalar fields with non-canonical kinetic terms often appear in particle physics. In general the action for such theories can be expressed as

$$
S=\int \mathrm{d}^{4} x \sqrt{-g}\left[\frac{1}{2 \kappa^{2}} R+P(\phi, X)\right]+S_{M},
$$


where $P(\phi, X)$ is a function in terms of a scalar field $\phi$ and its kinetic energy $X=-(1 / 2) g^{\mu \nu} \partial_{\mu} \phi \partial_{\nu} \phi$, and $S_{M}$ is a matter action. Even in the absence of the field potential $V(\phi)$ it is possible to realize the cosmic acceleration due to the kinetic energy $X$ [240]. The application of these theories to dark energy was first carried out by Chiba et al. [39]. In Ref. [40] this was extended to more general cases and the models based on the action (56) were named "k-essence". The action (56) includes a wide variety of theories listed below.

- (A) Low energy effective string theory

The action of low energy effective string theory in the presence of a higher-order derivative term $(\tilde{\nabla} \phi)^{4}$ is given by 241,242$]$

$$
S=\frac{1}{2 \kappa^{2}} \int \mathrm{d}^{4} \tilde{x} \sqrt{-\tilde{g}}\left[F(\phi) \tilde{R}+\omega(\phi)(\tilde{\nabla} \phi)^{2}+\alpha^{\prime} B(\phi)(\tilde{\nabla} \phi)^{4}+\mathcal{O}\left(\alpha^{\prime 2}\right)\right],
$$

which is derived by the expansion in terms of the Regge slope parameter $\alpha^{\prime}$ (this is related to the string mass scale $M_{s}$ via the relation $\left.M_{s}=\sqrt{2 / \alpha^{\prime}}\right)$. The scalar field $\phi$, dubbed a dilaton field, is coupled to the Ricci scalar $R$ with the strength $F(\phi)$. This frame is called the Jordan frame, in which the tilde is used in the action (57). Under a conformal transformation, $g_{\mu \nu}=F(\phi) \tilde{g}_{\mu \nu}$, we obtain the action in the Einstein frame [240]

$$
S_{E}=\int \mathrm{d}^{4} x \sqrt{-g}\left[\frac{1}{2 \kappa^{2}} R+K(\phi) X+L(\phi) X^{2}+\cdots\right],
$$

where $K(\phi)=3\left(F_{, \phi} / F\right)^{2}-2 \omega / F$ and $L(\phi)=2 \alpha^{\prime} B(\phi) / \kappa^{2}$.

- (B) Ghost condensate

The theories with a negative kinetic energy $-X$ generally suffers from the vacuum instability [243, 244], but the presence of the quadratic term $X^{2}$ can evade this problem. The model constructed in this vein is the ghost condensate model characterized by the Lagrangian [245]

$$
P=-X+X^{2} / M^{4},
$$

where $M$ is a constant. A more general version of this model, called the dilatonic ghost condensate [246], is

$$
P=-X+e^{\kappa \lambda \phi} X^{2} / M^{4},
$$

which is motivated by a dilatonic higher-order correction to the tree-level action [as we have discussed in the case $(\mathrm{A})$ ].

- (C) Tachyon

A tachyon field appears as an unstable mode of D-branes [non-Bogomol'nyi-Prasad-Sommerfield (non-BPS) branes]. The effective 4-dimensional Lagrangian is given by [247 249]

$$
P=-V(\phi) \sqrt{1-2 X},
$$

where $V(\phi)$ is a potential of the tachyon field $\phi$. While the tachyon model is difficult to be compatible with inflation in the early Universe because of the problem for ending inflation [250 252], one can use it for dark energy provided that the potential is shallower than $V(\phi)=V_{0} \phi^{-2}[253$ 258].

- (D) Dirac-Born-Infeld (DBI) theory

In the so-called "D-cceleration" mechanism in which a scalar field $\phi$ parametrizes a direction on the approximate Coulomb branch of the system in $\mathcal{N}=4$ supersymmetric Yang Mills theory, the field dynamics can be described by the DBI action for a probe D3-brane moving in a radial direction of the Anti de Sitter (AdS) space-time [259, 260]. The Lagrangian density with the field potential $V(\phi)$ is given by

$$
P=-f(\phi)^{-1} \sqrt{1-2 f(\phi) X}+f(\phi)^{-1}-V(\phi),
$$

where $f(\phi)$ is a warped factor of the AdS throat. In this theory one can realize the acceleration of the Universe even in the regime where $2 f(\phi) X$ is close to 1 . The application of this theory to dark energy has been carried out in Refs. 261 263]. 
For the theories with the action (56), the pressure $P_{\phi}$ and the energy density $\rho_{\phi}$ of the field are $P_{\phi}=P$ and $\rho_{\phi}=2 X P_{, X}-P$, respectively. The equation of state of k-essence is given by

$$
w_{\phi}=\frac{P_{\phi}}{\rho_{\phi}}=\frac{P}{2 X P_{, X}-P} .
$$

As long as the condition $\left|2 X P_{, X}\right| \ll|P|$ is satisfied, $w_{\phi}$ can be close to -1 . In the ghost condensate model (59)) we have

$$
w_{\phi}=\frac{1-X / M^{4}}{1-3 X / M^{4}}
$$

which gives $-1<w_{\phi}<-1 / 3$ for $1 / 2<X / M^{4}<2 / 3$. In particular the de Sitter solution $\left(w_{\phi}=-1\right)$ is realized at $X / M^{4}=1 / 2$. Since the field energy density is $\rho_{\phi}=M^{4} / 4$ at the de Sitter point, it is possible to explain the cosmic acceleration today for $M \simeq 10^{-3} \mathrm{eV}$.

In order to discuss stability conditions of k-essence in the Ultra-Violet (UV) regime, we decompose the field into the homogenous and perturbed parts as $\phi(t, \mathbf{x})=\phi_{0}(t)+\delta \phi(t, \mathbf{x})$ in the Minkowski background and derive the Lagrangian and the Hamiltonian for perturbations. The resulting second-order Hamiltonian reads [246]

$$
\delta H=\left(P_{, X}+2 X P_{, X X}\right) \frac{(\delta \dot{\phi})^{2}}{2}+P_{, X} \frac{(\nabla \delta \phi)^{2}}{2}-P_{, \phi \phi} \frac{(\delta \phi)^{2}}{2} .
$$

The term $P_{, \phi \phi}$ is related with the effective mass of the field, which is unimportant in the UV regime as long as the field is responsible for dark energy. The positivity of the first two terms in Eq. (65) leads to the following stability conditions

$$
P_{, X}+2 X P_{, X X} \geq 0, \quad P_{, X} \geq 0 .
$$

The phantom model with a negative kinetic energy $-X$ with a potential $V(\phi)$, i.e. $P=-X-V(\phi)$, do not satisfy the above conditions. Although the phantom model with $P=-X-V(\phi)$ can lead to the background cosmological dynamics allowed by SN Ia observations $\left(w_{\phi}<-1\right)$ [243, 264, 265], it suffers from a catastrophic particle production of ghosts and normal fields because of the instability of the vacuum [243, 244] (see Refs. [136, 266 280] for related works). This problem is overcome in the ghost condensate model (59) in which the conditions (66) are satisfied for $X / M^{4}>1 / 2^{3}$. Thus the successful k-essence models need to be constructed to be consistent with the conditions (66), while the field is responsible for dark energy under the condition $\left|2 X P_{, X}\right| \ll|P|$.

The propagation speed $c_{s}$ of the field is given by [282]

$$
c_{s}^{2}=\frac{P_{\phi, X}}{\rho_{\phi, X}}=\frac{P_{, X}}{P_{, X}+2 X P_{, X X}},
$$

which is positive under the conditions (66). The speed $c_{s}$ remains sub-luminal provided that

$$
P_{, X X}>0 \text {. }
$$

This condition is ensured for the models (59), (60), (61), and (62).

There are some k-essence models proposed to solve the coincidence problem of dark energy. One example is [40, 41]

$$
P=\frac{1}{\phi^{2}}\left(-2.01+2 \sqrt{1+X}+3 \cdot 10^{-17} X^{3}-10^{-24} X^{4}\right) .
$$

In these models the solutions can finally approach the accelerating phase even if they start from relatively large values of the k-essence energy density $\Omega_{\phi}$ in the radiation era. In such cases, however, there is a period in which the sound speed becomes superluminal before reaching the accelerated attractor [283]. Moreover it was shown that the basins of attraction of a radiation scaling solution in such models are restricted to be very small [284]. We stress that these problems arise only for the k-essence models constructed to solve the coincidence problem.

\footnotetext{
${ }^{3}$ It is possible that the dilatonic ghost condensate model crosses the cosmological constant boundary $w_{\phi}=-1$, but the quantum instability problem is present in the region $w_{\phi}<-1$. This crossing does not occur for the single-field k-essence Lagrangian with a linear function of $X$ [281].
} 


\section{Coupled dark energy}

Since the energy density of dark energy is the same order as that of dark matter in the present Universe, this implies that dark energy may have some relation with dark matter. In this section we discuss the cosmological viability of coupled dark energy models and related topics such as scaling solutions, the chameleon mechanism, and varying $\alpha$.

\section{The coupling between dark energy and dark matter}

In the flat FLRW cosmological background, a general coupling between dark energy (with energy density $\rho_{\text {DE }}$ and equation of state $w_{\mathrm{DE}}$ ) and dark matter (with energy density $\rho_{m}$ ) may be described by the following equations

$$
\begin{aligned}
\dot{\rho}_{\mathrm{DE}}+3 H\left(1+w_{\mathrm{DE}}\right) \rho_{\mathrm{DE}} & =-\beta, \\
\dot{\rho}_{m}+3 H \rho_{m} & =+\beta,
\end{aligned}
$$

where $\beta$ is the rate of the energy exchange in the dark sector.

There are several forms of couplings proposed so far. Two simple examples are given by

$$
\begin{aligned}
& \text { (A) } \beta=\kappa Q \rho_{m} \dot{\phi}, \\
& \text { (B) } \beta=\alpha H \rho_{m},
\end{aligned}
$$

where $Q$ and $\alpha$ are dimensionless constants. The coupling (A) arises in scalar-tensor theories after the conformal transformation to the Einstein frame [285 288]. In general the coupling $Q$ is field-dependent [289, 290], but Brans-Dicke theory [291] (including $f(R)$ gravity) gives rise to a constant coupling [292]. The coupling (B) is more phenomenological, but this form is useful to place observational bounds from the cosmic expansion history. Several authors studied other couplings of the forms $\beta=\left(\alpha_{m} \rho_{m}+\alpha_{\mathrm{DE}} \rho_{\mathrm{DE}}\right) H$ [293 295], $\beta=\alpha \Omega_{\mathrm{DE}} H$ [296 298], and $\beta=\Gamma \rho_{m}$ [299 301]. See also Refs. [302 318] for related works.

\section{(A) The coupling (A)}

Let us consider the coupling (A) in the presence of a coupled quintessence field with the exponential potential (48). We assume that the coupling $Q$ is constant. Taking into account radiation uncoupled to dark energy $\left(\rho_{r} \propto a^{-4}\right)$ the Friedmann equation is given by $3 H^{2}=\kappa^{2}\left(\rho_{\mathrm{DE}}+\rho_{m}+\rho_{r}\right)$, where $\rho_{\mathrm{DE}}=\dot{\phi}^{2} / 2+V(\phi)$. Introducing the dimensionless variables $x_{1}=\kappa \dot{\phi} /(\sqrt{6} H), x_{2}=\kappa \sqrt{V} /(\sqrt{3} H)$, and $x_{3}=\kappa \sqrt{\rho_{r}} /(\sqrt{3} H)$ as in Sec. IVA we obtain

$$
x_{1}^{\prime}=-3 x_{1}+\frac{\sqrt{6}}{2} \lambda x_{2}^{2}+\frac{1}{2} x_{1}\left(3+3 x_{1}^{2}-3 x_{2}^{2}+x_{3}^{2}\right)-\frac{\sqrt{6}}{2} Q\left(1-x_{1}^{2}-x_{2}^{2}-x_{3}^{2}\right),
$$

and the same differential equations for $x_{2}$ and $x_{3}$ as given in Eqs. (46) and (47). For this dynamical system there is a scalar-field dominated fixed point given in Eq. (49) as well as the radiation point $\left(x_{1}, x_{2}, x_{3}\right)=(0,0,1)$. In the presence of the coupling $Q$, the standard matter era is replaced by a " $\phi$-matter-dominated epoch $(\phi \mathrm{MDE})$ " [287] characterized by

$$
\left(x_{1}, x_{2}, x_{3}\right)=(-\sqrt{6} Q / 3,0,0), \quad \Omega_{\phi}=2 Q^{2} / 3, \quad w_{\phi}=1 .
$$

Defining the effective equation of state

$$
w_{\text {eff }}=-1-2 \dot{H} /\left(3 H^{2}\right),
$$

one has $w_{\text {eff }}=2 Q^{2} / 3$ for the $\phi \mathrm{MDE}$, which is different from 0 in the uncoupled case. Provided that $2 Q^{2} / 3<1$, the $\phi \mathrm{MDE}$ is a saddle followed by the accelerated point (49) [287].

The evolution of the scale factor during the $\phi \mathrm{MDE}$ is given by $a \propto t^{2 /\left(3+2 Q^{2}\right)}$, which is different from that in the uncoupled quintessence. This leads to a change to the CMB shift parameter defined in Eq. (23). From the CMB likelihood analysis, the strength of the coupling is constrained to be $|Q|<0.1$ [287]. The evolution of matter density perturbations is also subject to change by the effect of the coupling. Under a quasi-static approximation on sub-horizon scales the matter perturbation $\delta_{m}$ obeys the following equation [319, 320]

$$
\ddot{\delta}_{m}+(2 H+Q \dot{\phi}) \dot{\delta}_{m}-4 \pi G_{\text {eff }} \rho_{m} \delta_{m} \simeq 0,
$$


where the effective gravitational coupling is given by $G_{\text {eff }}=\left(1+2 Q^{2}\right) G$. During the $\phi \mathrm{MDE}$ one can obtain the analytic solution to Eq. (77), as $\delta_{m} \propto a^{1+2 Q^{2}}$. Hence the presence of the coupling $Q$ leads to a larger growth rate relative to the uncoupled quintessence. We can parameterize the growth rate of matter perturbations, as [321]

$$
f \equiv \frac{\dot{\delta}_{m}}{H \delta_{m}}=\left(\Omega_{m}\right)^{\gamma},
$$

where $\Omega_{m} \equiv \kappa^{2} \rho_{m} /\left(3 H^{2}\right)$ is the density parameter of non-relativistic matter. In the $\Lambda$ CDM model the growth index $\gamma$ can be approximately given by $\gamma \simeq 0.55[322$, 323]. In the coupled quintessence the growth rate can be fitted to the numerical solution by the formula $f=\left(\Omega_{m}\right)^{\gamma}\left(1+c Q^{2}\right)$, where $c=2.1$ and $\gamma=0.56$ are the best-fit values [324]. Using the galaxy and Lyman- $\alpha$ power spectra, the growth index $\gamma$ and the coupling $Q$ are constrained to be $\gamma=0.6_{-0.3}^{+0.4}$ and $|Q|<0.52$ (95\% confidence level), respectively. This is weaker than the bound coming from the CMB constraint [324]. We also note that the equation for matter perturbations has been derived for the coupled k-essence scenario with a field-dependent coupling $Q(\phi)$ [325, 326]. In principle it is possible to reconstruct the coupling from observations if the evolution of matter perturbations is known accurately [327].

There is another interesting coupled dark energy scenario called mass varying neutrino 328 332] in which the mass $m_{\nu}$ of the neutrino depends on the quintessence field $\phi$. The energy density $\rho_{\nu}$ and the pressure $P_{\nu}$ of neutrinos can be determined by assuming a Fermi-Dirac distribution with the neglect of the chemical potential. It then follows that the field $\phi$ obeys the equation of motion [331]

$$
\ddot{\phi}+3 H \dot{\phi}+V_{, \phi}=-\kappa Q(\phi)\left(\rho_{\nu}-3 P_{\nu}\right),
$$

where $Q(\phi) \equiv \frac{\mathrm{d} \ln m_{\nu}(\phi)}{\mathrm{d} \phi}$. In the relativistic regime in which the neutrino mass $m_{\nu}$ is much smaller than the neutrino temperature $T_{\nu}$, the r.h.s. of Eq. (79) is suppressed because of the relation $\rho_{\nu} \simeq 3 P_{\nu}$. In the non-relativistic regime with $m_{\nu} \gg T_{\nu}$ the pressure $P_{\nu}$ is much smaller than the energy density $\rho_{\nu}$, in which case the field equation (79) mimics Eq. (70) with the coupling (A). In the mass varying neutrino scenario the field-dependent mass of neutrinos determines the strength of the coupling $Q(\phi)$. In the non-relativistic regime the neutrino energy density is approximately given by $\rho_{\nu} \simeq n_{\nu} m_{\nu}(\phi)$, where $n_{\nu}$ is the number density of neutrinos. Then the effective potential of the field is given by $V_{\text {eff }}(\phi) \simeq V(\phi)+n_{\nu} m_{\nu}(\phi)$, which gives rise to a minimum for a runaway quintessence potential $V(\phi)$. Since the field equation of state in this regime is $w_{\phi} \simeq-V(\phi) /\left[V(\phi)+n_{\nu} m_{\nu}(\phi)\right]$, one has $w_{\phi} \simeq-1$ for $n_{\nu} m_{\nu} \ll V(\phi)$. See Refs. [333 338] for a number of cosmological consequences of the mass varying neutrino scenario.

\section{(B) The coupling (B)}

Let us consider the coupling (B) given in Eq. (73). For constant $\delta$ one can integrate Eq. (71) as $\rho_{m}=\rho_{m}^{(0)}(1+z)^{3-\alpha}$, where $z$ is a redshift. If the equation of state $w_{\mathrm{DE}}$ is constant, one obtains the following integrated solution for Eq. (70):

$$
\rho_{\mathrm{DE}}=\rho_{\mathrm{DE}}^{(0)}(1+z)^{3\left(1+w_{\mathrm{DE}}\right)}+\rho_{m}^{(0)} \frac{\alpha}{\alpha+3 w_{\mathrm{DE}}}\left[(1+z)^{3\left(1+w_{\mathrm{DE}}\right)}-(1+z)^{3-\alpha}\right] .
$$

The Friedmann equation, $3 H^{2}=\kappa^{2}\left(\rho_{\mathrm{DE}}+\rho_{m}\right)$, gives the parametrization for the normalized Hubble parameter $E(z)=H(z) / H_{0}$, as

$$
E^{2}(z)=\Omega_{\mathrm{DE}}^{(0)}(1+z)^{3\left(1+w_{\mathrm{DE}}\right)}+\frac{1-\Omega_{\mathrm{DE}}^{(0)}}{\alpha+3 w_{\mathrm{DE}}}\left[\alpha(1+z)^{3\left(1+w_{\mathrm{DE}}\right)}+3 w_{\mathrm{DE}}(1+z)^{3-\alpha}\right] .
$$

This parametrization can be used to place observational constraints on the coupling $\alpha$. The combined data analysis using the observational data of the 5-year Supernova Legacy Survey (SNLS) [13], the CMB shift parameter from the 3-year WMAP [20], and the BAO [23] shows that $\alpha$ and $w_{\mathrm{DE}}$ are constrained to be $-0.08<\alpha<0.03$ and $-1.16<w_{\mathrm{DE}}<-0.91$ (95\% confidence level) [298]. In Ref. [300] it was shown that, for constant $w_{\mathrm{DE}}$, cosmological perturbations are subject to non-adiabatic instabilities in the early radiation era. This problem can be alleviated by considering the time-dependent $w_{\mathrm{DE}}$ satisfying the condition $w_{\mathrm{DE}}>-4 / 5$ at early times [317].

It is also possible to extend the analysis to the case in which the coupling $\alpha$ varies in time. Dalal et al. 296] assumed the scaling relation, $\rho_{\mathrm{DE}} / \rho_{m}=\left(\rho_{\mathrm{DE}}^{(0)} / \rho_{m}^{(0)}\right) a^{\xi}$, where $\xi$ is a constant. For constant $w_{\mathrm{DE}}$ the coupling $\alpha$ is expressed in the form

$$
\alpha(z)=\frac{\alpha_{0}}{\Omega_{\mathrm{DE}}^{(0)}+\left(1-\Omega_{\mathrm{DE}}^{(0)}\right)(1+z)^{\xi}},
$$


where $\alpha_{0}=-\left(\xi+3 w_{\mathrm{DE}}\right) \Omega_{\mathrm{DE}}^{(0)}$. If $\xi>0, \alpha(z)$ decreases for higher $z$. In this case the Hubble parameter can be parametrized as

$$
E^{2}(z)=(1+z)^{3}\left[1-\Omega_{\mathrm{DE}}^{(0)}+\Omega_{\mathrm{DE}}^{(0)}(1+z)^{-\xi}\right]^{-3 w_{\mathrm{DE}} / \xi}
$$

The combined data analysis using the SNLS, WMAP 3-year, and the BAO gives the bounds $-0.4<\alpha_{0}<0.1$ and $-1.18<w_{\mathrm{DE}}<-0.91$ (95\% confidence level) [298]. The $\Lambda \mathrm{CDM}$ model $\left(\alpha=0, w_{\mathrm{DE}}=-1\right)$ remains a good fit to the data.

\section{Coupled dark energy and coincidence problem}

In the coupled quintessence with an exponential potential $\left(V(\phi)=V_{0} e^{-\kappa \lambda \phi}\right.$ with $\left.\lambda>0\right)$ the $\phi$ MDE scaling solution with $\Omega_{\phi}=2 Q^{2} / 3=$ constant replaces the standard matter era. In this model there is another scaling solution given by $[287, \underline{306}]$

$$
\begin{aligned}
& \left(x_{1}, x_{2}, x_{3}\right)=\left(\frac{\sqrt{6}}{2(Q+\lambda)}, \sqrt{\frac{2 Q(Q+\lambda)+3}{2(Q+\lambda)^{2}}}, 0\right), \quad \Omega_{\phi}=\frac{Q(Q+\lambda)+3}{(Q+\lambda)^{2}}, \\
& w_{\phi}=-\frac{Q(Q+\lambda)}{Q(Q+\lambda)+3}, \quad w_{\text {eff }}=-\frac{Q}{Q+\lambda} .
\end{aligned}
$$

In the presence of the coupling $Q$ the condition for the late-time cosmic acceleration, $w_{\text {eff }}<-1 / 3$, is satisfied for $Q>\lambda / 2>0$ or $Q<-\lambda<0$. Then the scaling solution (84) can give rise to the global attractor with $\Omega_{\phi} \simeq 0.7$. The $\phi \mathrm{MDE}$ solution (75) followed by the accelerated scaling solution (84) may be used for alleviating the coincidence problem, because dark energy and dark matter follow the same scaling relation from the end of the radiation era. In Ref. [287] it was shown, however, that the coupled quintessence with an exponential potential does not allow for such cosmological evolution for the viable parameter space in the $(Q, \lambda)$ plane consistent with observational constraints.

It is possible to extend the analysis to coupled k-essence models described by the action (56) in the presence of the coupling (72). From the requirement that the density parameter $\Omega_{\phi}(\neq 0)$ and the equation of state $w_{\phi}$ are constants to realize scaling solutions, one can show that the Lagrangian density takes the following form [246, 339]

$$
P=X g\left(X e^{\kappa \lambda \phi}\right),
$$

where $\lambda$ is a constant, and $g$ is an arbitrary function in terms of $Y \equiv X e^{\kappa \lambda \phi}$. The result (85) is valid for constant $Q$, but it can be generalized to a field-dependent coupling $Q(\phi)$ [340]. The quintessence with an exponential potential $\left(P=X-c e^{-\kappa \lambda \phi}\right)$ corresponds to $g(Y)=1-c / Y$. Since the dilatonic ghost condensation model (60) corresponds to $g(Y)=-1+Y / M^{4}$, this model has a scaling solution. We can also show that the tachyon field with the Lagrangian density $P=-V(\phi) \sqrt{1-2 X}$ also has a scaling solution for the potential $V(\phi) \propto \phi^{-2}$ [257]. Even when the Hubble parameter squared $H^{2}$ is proportional to the energy density $\rho^{n}$, it is possible to obtain the Lagrangian density having scaling solutions in the form $P=X^{1 / n} g\left(X e^{n \kappa \lambda \phi}\right)[339]$. The cosmological dynamics of scaling solutions in such cases (including the high-energy regime [341, 342] in the Randall-Sundrum scenario [343, 344]) have been discussed by a number of authors 345 347.

For the Lagrangian density (85) there are two fixed points relevant to dark energy. Defining the dimensionless variables $x \equiv \dot{\phi} /(\sqrt{6} H)$ and $y \equiv e^{-\lambda \phi / 2} /(\sqrt{3} H)$ (in the unit of $\left.\kappa^{2}=1\right)$, they are given by [348]

- (A) Scalar-field dominated solution

$$
x_{A}=\frac{\lambda}{\sqrt{6} P_{, X}}, \quad \Omega_{\phi}=1, \quad w_{\mathrm{eff}}=w_{\phi}=-1+\frac{\lambda^{2}}{3 P_{, X}} .
$$

- (B) Scaling solution

$$
\begin{aligned}
& x_{B}=\frac{\sqrt{6}}{2(Q+\lambda)}, \quad \Omega_{\phi}=\frac{Q(Q+\lambda)+3 P_{, X}}{(Q+\lambda)^{2}} . \\
& w_{\text {eff }}=-\frac{Q}{Q+\lambda}, \quad w_{\phi}=-\frac{Q(Q+\lambda)}{Q(Q+\lambda)+3 P_{, X}} .
\end{aligned}
$$


The points (A) and (B) are responsible for the cosmic acceleration for (A) $\lambda^{2} / P_{, X}<2$ and (B) $Q>\lambda / 2>0$ or $Q<-\lambda<0$, respectively. From the stability analysis about the fixed points it follows that, when the point (A) is stable, the point (B) is not stable, and vice versa [348].

The $\phi \mathrm{MDE}$ corresponds to a fixed point at which the kinetic energy of the field dominates over the potential energy, i.e. $x \neq 0$ and $y=0$. Since the quantity $Y=X e^{\kappa \lambda \phi}$ can be expressed as $Y=x^{2} / y^{2}$, the function $g(Y)$ cannot be singular at $y=0$ for the existence of the $\phi \mathrm{MDE}$. Then the function $g(Y)$ should be expanded in negative powers of $Y$, i.e.

$$
g(Y)=c_{0}+\sum_{n>0} c_{n} Y^{-n}=c_{0}+\sum_{n>0} c_{n}\left(y^{2} / x^{2}\right)^{n}
$$

which includes the quintessence with an exponential potential. For this form of $g(Y)$, there is the following $\phi \mathrm{MDE}$ point (C):

$$
\left(x_{C}, y_{C}\right)=\left(-\frac{\sqrt{6} Q}{3 c_{0}}, 0\right), \quad \Omega_{\phi}=w_{\mathrm{eff}}=\frac{2 Q^{2}}{3 c_{0}}, \quad w_{\phi}=1
$$

together with the purely kinetic point $(x, y)=\left( \pm 1 / \sqrt{c_{0}}, 0\right)$ and $\Omega_{\phi}=1$ for $c_{0}>0$.

An ideal cosmological trajectory that alleviates the coincidence problem of dark energy should be the $\phi \mathrm{MDE}(\mathrm{C})$ followed by the point (B). However, it was shown in Ref. [340] that such a trajectory is not allowed because the solutions cannot cross the singularity at $x=0$ as well as another singularity associated with the sound speed. For example, when $c_{0}>0$, we find that $x_{B}>0$ and $x_{C}<0$ for $Q>\lambda / 2>0$, whereas $x_{B}<0$ and $x_{C}>0$ for $Q<-\lambda<0$. These points are separated between the line $x=0$ at which the function (88) diverges. The $\phi$ MDE solution chooses the accelerated point (A) as a final attractor. The above discussion shows that the coincidence problem is difficult to be solved even for the general Lagrangian density (85) that has scaling solutions. This problem mainly comes from the fact that a large coupling $Q$ required for the existence of a viable scaling solution (B) is not compatible with a small coupling $Q$ required for the existence of the $\phi \mathrm{MDE}$. We need a rapidly growing coupling to realize such a transition [349].

\section{Chameleon mechanism}

If a scalar field $\phi$ is coupled to baryons as well as dark matter, this gives rise to a fifth force interaction that can be constrained experimentally. A large coupling of the order of unity arises in modified gravity theories as well as superstring theories. In such cases we need to suppress such a strong interaction with baryons for the compatibility with local gravity experiments. There is a way to screen the fifth force under the so-called chameleon mechanism [350, 351] in which the field mass is different depending on the matter density in the surrounding environment. If the field is sufficiently heavy in the regions of high density, a spherically symmetric body can have a "thin-shell" around its surface such that the effective coupling between the field and matter is suppressed outside the body.

The action of a chameleon scalar field $\phi$ with a potential $V(\phi)$ is given by

$$
S=\int \mathrm{d}^{4} x \sqrt{-g}\left[\frac{1}{2 \kappa^{2}} R-\frac{1}{2} g^{\mu \nu} \partial_{\mu} \phi \partial_{\nu} \phi-V(\phi)\right]+\int \mathrm{d}^{4} x \mathcal{L}_{M}\left(g_{\mu \nu}^{(i)}, \Psi_{M}^{(i)}\right),
$$

where $g$ is the determinant of the metric $g_{\mu \nu}$ (in the Einstein frame) and $\mathcal{L}_{M}$ is a matter Lagrangian with matter fields $\Psi_{M}^{(i)}$ coupled to a metric $g_{\mu \nu}^{(i)}$. The metric $g_{\mu \nu}^{(i)}$ is related to the Einstein frame metric $g_{\mu \nu}$ via $g_{\mu \nu}^{(i)}=e^{2 \kappa Q_{i} \phi} g_{\mu \nu}$, where $Q_{i}$ are the strengths of the couplings for each matter component with the field $\phi$. The typical field potential is chosen to be of the runaway type ( $\operatorname{such}$ as $V(\phi)=M^{4+n} \phi^{-n}$ ). We also restrict the form of the potential such that $\left|V_{, \phi}\right| \rightarrow \infty$ as $\phi \rightarrow 0$.

Varying the action (90) with respect to $\phi$, we obtain the field equation

$$
\square \phi-V_{, \phi}=-\sum_{i} \kappa Q_{i} e^{4 \kappa Q_{i} \phi} g_{(i)}^{\mu \nu} T_{\mu \nu}^{(i)},
$$

where $T_{\mu \nu}^{(i)}=-\left(2 / \sqrt{-g^{(i)}}\right) \delta \mathcal{L}_{M} / \delta g_{(i)}^{\mu \nu}$ is the stress-energy tensor for the $i$-th form of matter. For non-relativistic matter we have $g_{(i)}^{\mu \nu} T_{\mu \nu}^{(i)}=-\tilde{\rho}_{i}$, where $\tilde{\rho}_{i}$ is an energy density. It is convenient to introduce the energy density $\rho_{i} \equiv \tilde{\rho}_{i} e^{3 \kappa Q_{i} \phi}$, which is conserved in the Einstein frame. In the following, let us consider the case in which the couplings $Q_{i}$ are the 
same for all species. i.e. $Q_{i}=Q$. In a spherically symmetric space-time under the weak gravitational background (i.e. neglecting the backreaction of gravitational potentials), Eq. (91) reads

$$
\frac{\mathrm{d}^{2} \phi}{\mathrm{d} r^{2}}+\frac{2}{r} \frac{\mathrm{d} \phi}{\mathrm{d} r}=\frac{\mathrm{d} V_{\text {eff }}}{\mathrm{d} \phi},
$$

where $r$ is a distance from the center of symmetry, and $V_{\text {eff }}$ is the effective potential given by

$$
V_{\text {eff }}(\phi)=V(\phi)+e^{\kappa Q \phi} \rho,
$$

and $\rho \equiv \sum_{i} \rho_{i}$. For the runaway potential with $V_{, \phi}<0$ the positive coupling $Q$ leads to a minimum of the effective potential. In $f(R)$ gravity the negative coupling $(Q=-1 / \sqrt{6})$ gives rise to a minimum for the potential with $V_{, \phi}>0$ (as we will see in Sec. VA3).

We assume that a spherically symmetric body has a constant density $\rho=\rho_{A}$ inside the body $\left(r<r_{c}\right)$ and that the energy density outside the body $\left(r>r_{c}\right)$ is $\rho=\rho_{B}$. The mass $M_{c}$ of the body and the gravitational potential $\Phi_{c}$ at the radius $r_{c}$ are given by $M_{c}=(4 \pi / 3) r_{c}^{3} \rho_{A}$ and $\Phi_{c}=G M_{c} / r_{c}$, respectively. The effective potential $V_{\text {eff }}(\phi)$ has two minima at the field values $\phi_{A}$ and $\phi_{B}$ satisfying $V_{\text {eff }}^{\prime}\left(\phi_{A}\right)=0$ and $V_{\text {eff }}^{\prime}\left(\phi_{B}\right)=0$, respectively. The former corresponds to the region with a high density that gives rise to a heavy mass squared $m_{A}^{2} \equiv V_{\text {eff }}^{\prime \prime}\left(\phi_{A}\right)$, whereas the latter to the lower density region with a lighter mass squared $m_{B}^{2} \equiv V_{\text {eff }}^{\prime \prime}\left(\phi_{B}\right)$. When we consider the "dynamics" of the field $\phi$ according to Eq. (92) we need to consider the inverted effective potential $\left(-V_{\text {eff }}\right)$ having two maxima at $_{\phi}=\phi_{A}$ and $\phi=\phi_{B}$.

The boundary conditions for the field are given by $\frac{\mathrm{d} \phi}{\mathrm{d} r}(r=0)=0$ and $\phi(r \rightarrow \infty)=\phi_{B}$. The field $\phi$ is at rest at $r=0$ and begins to roll down the potential when the matter-coupling term $\kappa Q \rho_{A} e^{\kappa Q \phi}$ becomes important at a radius $r_{1}$ in Eq. (92). As long as $r_{1}$ is close to $r_{c}$ so that $\Delta r_{c} \equiv r_{c}-r_{1} \ll r_{c}$, the body has a thin-shell inside the body. Since the field acquires a sufficient kinetic energy in the thin-shell regime $\left(r_{1}<r<r_{c}\right)$, it climbs up the potential hill outside the body $\left(r>r_{c}\right)$. The field profile can be obtained by matching the solutions of Eq. (92) at the radius $r=r_{1}$ and $r=r_{c}$. Neglecting the mass term $m_{B}$, we obtain the thin-shell field profile outside the body [350 352]

$$
\phi(r) \simeq \phi_{B}-\frac{2 Q_{\mathrm{eff}}}{\kappa} \frac{G M_{c}}{r}
$$

where

$$
Q_{\mathrm{eff}}=3 Q \epsilon_{\mathrm{th}}, \quad \epsilon_{\mathrm{th}} \equiv \frac{\kappa\left(\phi_{B}-\phi_{A}\right)}{6 Q \Phi_{c}}
$$

Here $\epsilon_{\mathrm{th}}$ is called the thin-shell parameter. Under the conditions $\Delta r_{c} / r_{c} \ll 1$ and $1 /\left(m_{A} r_{c}\right) \ll 1$, the thin-shell parameter is approximately given by $\epsilon_{\mathrm{th}} \simeq \Delta r_{c} / r_{c}+1 /\left(m_{A} r_{c}\right)$ [352]. As long as $\epsilon_{\mathrm{th}} \ll 1$ the amplitude of the effective coupling $Q_{\text {eff }}$ can be much smaller than 1 . Hence it is possible for the large coupling models $(|Q|=\mathcal{O}(1))$ to be consistent with local gravity experiments if the body has a thin-shell.

Let us study the constraint on the thin-shell parameter from the possible violation of the equivalence principle (EP). The tightest bound comes from the solar system tests of weak EP using the free-fall acceleration of Moon $\left(a_{\mathrm{M} o o n}\right)$ and Earth $\left(a_{\oplus}\right)$ toward Sun [351]. The experimental bound on the difference of two accelerations is given by [353, 354]

$$
\frac{\left|a_{\text {Moon }}-a_{\oplus}\right|}{\left(a_{\text {Moon }}+a_{\oplus}\right) / 2}<10^{-13} .
$$

If Earth, Sun, and Moon have thin-shells, the field profiles outside the bodies are given by Eq. (94) with the replacement of corresponding quantities. The acceleration induced by a fifth force with the field profile $\phi(r)$ and the effective coupling $Q_{\text {eff }}$ is $a^{\text {fifth }}=\left|Q_{\text {eff }} \nabla \phi(r)\right|$. Using the thin-shell parameter $\epsilon_{\mathrm{th}, \oplus}$ for Earth, the accelerations $a_{\oplus}$ and $a_{\mathrm{Moon}}$ toward Sun (mass $M_{\odot}$ ) are [351]

$$
\begin{aligned}
a_{\oplus} & \simeq \frac{G M_{\odot}}{r^{2}}\left[1+18 Q^{2} \epsilon_{\mathrm{th}, \oplus}^{2} \frac{\Phi_{\oplus}}{\Phi_{\odot}}\right], \\
a_{\mathrm{Moon}} & \simeq \frac{G M_{\odot}}{r^{2}}\left[1+18 Q^{2} \epsilon_{\mathrm{th}, \oplus}^{2} \frac{\Phi_{\oplus}^{2}}{\Phi_{\odot} \Phi_{\mathrm{Moon}}}\right],
\end{aligned}
$$

where $\Phi_{\odot} \simeq 2.1 \times 10^{-6}, \Phi_{\oplus} \simeq 7.0 \times 10^{-10}$, and $\Phi_{\text {Moon }} \simeq 3.1 \times 10^{-11}$ are the gravitational potentials of Sun, Earth and Moon, respectively. Hence the condition (96) translates into

$$
\epsilon_{\mathrm{th}, \oplus}<8.8 \times 10^{-7} /|Q|
$$


Since the condition $\left|\phi_{B}\right| \gg\left|\phi_{A}\right|$ is satisfied for the field potentials under consideration, one has $\epsilon_{\mathrm{th}, \oplus} \simeq \kappa \phi_{B} /\left(6 Q \Phi_{\oplus}\right)$ from Eq. (95). Then the condition (99) translates into

$$
\left|\kappa \phi_{B, \oplus}\right|<3.7 \times 10^{-15} .
$$

For example, let us consider the inverse power-law potential $V(\phi)=M^{4+n} \phi^{-n}$. In this case we have $\phi_{B, \oplus}=$ $\left[(n / Q)\left(M_{\mathrm{pl}}^{4} / \rho_{B}\right)\left(M / M_{\mathrm{pl}}\right)^{n+4}\right]^{1 /(n+1)} M_{\mathrm{pl}}$, where we recovered the reduced Planck mass $M_{\mathrm{pl}}=1 / \kappa$. For $n$ and $Q$ of the order of unity, the constraint (100) gives $M<10^{-(15 n+130) /(n+4)} M_{\mathrm{pl}}$. When $n=1$, for example, one has $M<10^{-2} \mathrm{eV}$. If the same potential is responsible for dark energy, the mass $M$ is constrained to be larger than this value [355]. For the potential $V(\phi)=M^{4} \exp \left(M^{n} / \phi^{n}\right)$, however, we have that $V(\phi) \approx M^{4}+M^{4+n} \phi^{-n}$ for $\phi>M$, which is responsible for dark energy for $M \approx 10^{-3} \mathrm{eV}$. This can be compatible with the mass scale $M$ constrained by (100) [355]. See Refs. [356 358, 358, 359, 359 363] for a number of cosmological and experimental aspects of the chameleon field.

\section{4. $\quad$ Varying $\alpha$}

So far we have discussed the coupling between dark energy and non-relativistic matter. In this section we discuss the case in which dark energy is coupled to an electromagnetic field. In fact, a temporal variation of the effective fine structure "constant" $\alpha$ has been reported by a number of authors. The variation of $\alpha$ constrained by the Oklo natural fission reactor is given by $-0.9 \times 10^{-7}<\Delta \alpha / \alpha \equiv\left(\alpha-\alpha_{0}\right) / \alpha_{0}<1.2 \times 10^{-7}$ at the redshift $z \approx 0.16$, where $\alpha_{0}$ is the value of $\alpha$ today [364]. From the absorption line spectra of distant quasars we have the constraints $\Delta \alpha / \alpha=(-0.574 \pm 0.102) \times 10^{-5}$ over the redshift range $0.2<z<3.7[365,366]$ and $\Delta \alpha / \alpha=(-0.06 \pm 0.06) \times 10^{-5}$ for $0.4<z<2.3$ [367]. Although the possibility of systematic errors still remains [368], this may provide important implications for the existence of a light scalar field related with dark energy. by

The Lagrangian density describing such a coupling between the field $\phi$ and the electromagnetic field $F_{\mu \nu}$ is given

$$
\mathcal{L}_{F}(\phi)=-\frac{1}{4} B_{F}(\phi) F_{\mu \nu} F^{\mu \nu}
$$

The coupling of the form $B_{F}(\phi)=e^{-\zeta \kappa\left(\phi-\phi_{0}\right)}$ was originally introduced by Bekenstein [369], where $\zeta$ is a coupling constant and $\phi_{0}$ is the field value today. There are also other choices of the coupling, see e.g., Refs. [370 377]. The fine structure "constant" $\alpha$ is inversely proportional to $B_{F}(\phi)$, so that this can be expressed as $\alpha=\alpha_{0} / B_{F}(\phi)$, where $\alpha_{0}$ is the present value. The exponential coupling $B_{F}(\phi)=e^{-\zeta \kappa\left(\phi-\phi_{0}\right)}$ has a linear dependence $B_{F}(\phi) \simeq 1-\zeta \kappa\left(\phi-\phi_{0}\right)$ in the regime $\left|\zeta \kappa\left(\phi-\phi_{0}\right)\right| \ll 1$, so that the variation of $\alpha$ is given by

$$
\frac{\Delta \alpha}{\alpha}=\frac{\alpha-\alpha_{0}}{\alpha_{0}} \simeq \zeta \kappa\left(\phi-\phi_{0}\right) .
$$

Using the constraint $\Delta \alpha / \alpha \simeq-10^{-5}$ around $z=3[366]$ obtained from quasar absorption lines, the coupling $\zeta$ can be expressed as

$$
\zeta \simeq-\frac{10^{-5}}{\kappa \phi(z=3)-\kappa \phi(z=0)} .
$$

Let us consider the case in which the scalar field has a power-law dependence in terms of the scale factor $a$, i.e.

$$
\phi=\phi_{0} a^{p}=\phi_{0}(1+z)^{-p} .
$$

In fact, in the so-called tracking regime of the matter-dominated era [38], the inverse power-law potential $V(\phi)=$ $M^{4+n} \phi^{-n}$ gives rise to a constant field equation of state: $w_{\phi}=-2 /(n+2)$, which corresponds to the field evolution (104) with $p=3 /(n+2)$. Using Eq. (104), the coupling $\zeta$ in Eq. (103) reads

$$
\zeta \simeq \frac{10^{-5}}{1-4^{-p}}\left(\kappa \phi_{0}\right)^{-1} .
$$

Since $\kappa \phi_{0}$ is of the order of unity in order to realize the present cosmic acceleration [38], the coupling $\zeta$ is constrained to be $\zeta \approx 10^{-5}$ for $p$ of the order of 1 .

The above discussion is valid for the potentials having the solution (104) in the tracking regime. The variation of $\alpha$ for other quintessence potentials was discussed in Ref. [376]. There is also a k-essence model in which a tachyon field is coupled to electromagnetic fields [378]. 


\section{Unified models of dark energy and dark matter}

There are a number of works to explain the origin of dark energy and dark matter using a single fluid or a single scalar field. Let us first discuss the generalized Chaplygin gas (GCG) model as an example of a single fluid model [44, 45]. In this model the pressure $P$ of the perfect fluid is related to its energy density $\rho$ via

$$
P=-A \rho^{-\alpha}
$$

where $A$ is a positive constant. The original Chaplygin gas model corresponds to $\alpha=1$ [44].

Plugging the relation (106) into the continuity equation $\dot{\rho}+3 H(\rho+P)=0$, we obtain the integrated solution

$$
\rho(t)=\left[A+\frac{B}{a^{3(1+\alpha)}}\right]^{1 /(1+\alpha)}
$$

where $B$ is an integration constant. In the early epoch $(a \ll 1)$ the energy density evolves as $\rho \propto a^{-3}$, which means that the fluid behaves as dark matter. In the late epoch $(a \gg 1)$ the energy density approaches a constant value $A^{1 /(a+\alpha)}$ and hence the fluid behaves as dark energy. A fluid with the generalized Chaplygin gas therefore interpolates between dark matter and dark energy.

Although this model is attractive to provide unified description of two dark components, it is severely constrained by the matter power spectrum in large-scale structure. The gauge-invariant matter perturbation $\delta_{m}$ with a comoving wavenumber $k$ obeys the following equation of motion [46]

$$
\ddot{\delta}_{m}+\left(2+3 c_{s}^{2}-6 w\right) H \dot{\delta}_{m}-\left[\frac{3}{2} H^{2}\left(1-6 c_{s}^{2}-3 w^{2}+8 w\right)-\left(\frac{c_{s} k}{a}\right)^{2}\right] \delta_{m}=0
$$

where $w=P / \rho$ is the fluid equation of state, and $c_{s}$ is the sound speed given by

$$
c_{s}^{2}=\frac{\mathrm{d} P}{\mathrm{~d} \rho}=-\alpha w
$$

Since $w \rightarrow 0$ and $c_{s}^{2} \rightarrow 0$ in the limit $z \gg 1$, the sound speed is much smaller than unity in the deep matter era and starts to grow around the end of it. Since $w$ is negative, $c_{s}^{2}$ is positive for $\alpha>0$ and negative for $\alpha<0$.

From Eq. (108) the perturbations satisfying the following condition grow via the gravitational instability

$$
\left|c_{s}^{2}\right|<\frac{3}{2}\left(\frac{a H}{k}\right)^{2}
$$

When $\left|c_{s}^{2}\right|>(3 / 2)(a H / k)^{2}$, the perturbations exhibit either rapid growth or damped oscillations depending on the sign of $c_{s}^{2}$. The violation of the condition (110) mainly occurs around the present epoch in which $|w|$ is of the order of unity and hence $\left|c_{s}^{2}\right| \sim|\alpha|$. The smallest scale relevant to the galaxy matter power spectrum in the linear regime

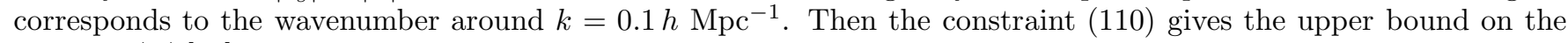
values of $|\alpha|$ [46]:

$$
|\alpha| \lesssim 10^{-5}
$$

Hence the generalized Chaplygin gas model is hardly distinguishable from the $\Lambda$ CDM model. In particular the original Chaplygin gas model $(\alpha=1)$ is excluded from the observations of large-scale structure. Although nonlinear clustering may change the evolution of perturbations in this model [379, 380], it is unlikely that the constraint (111) is relaxed significantly.

The above conclusion comes from the fact that in the Chaplygin gas model the sound speed is too large to match with observations. There is a way to avoid this problem by adding a non-adiabatic contribution to Eq. (108) to make $c_{s}$ vanish [381]. It is also possible to construct unified models of dark energy and dark matter using a purely kinetic scalar field [382]. Let us consider k-essence models in which the Lagrangian density $P(X)$ has an extremum at some value $X=X_{0}$, e.g. 382 ]

$$
P=P_{0}+P_{2}\left(X-X_{0}\right)^{2}
$$

The pressure $P_{\phi}=P$ and the energy density $\rho_{\phi}=2 X P_{, X}-P$ satisfy the continuity equation $\dot{\rho}_{\phi}+3 H\left(\rho_{\phi}+P_{\phi}\right)=0$, i.e.

$$
\left(P_{, X}+2 X P_{, X X}\right) \dot{X}+6 H P_{, X} X=0 .
$$


The solution around $X=X_{0}$ can be derived by introducing a small parameter $\epsilon=\left(X-X_{0}\right) / X_{0}$. Plugging Eq. (112) into Eq. (113), we find that $\epsilon$ satisfies the equation $\dot{\epsilon}=-3 H \epsilon$ at linear order. Hence we obtain the solution $X=X_{0}\left[1+\epsilon_{1}\left(a / a_{1}\right)^{-3}\right]$, where $\epsilon_{1}$ and $a_{1}$ are constants. The validity of the above approximation demands that $\epsilon_{1}\left(a / a_{1}\right)^{-3} \ll 1$. Since $P_{\phi} \simeq P_{0}$ and $\rho_{\phi} \simeq-P_{0}+4 P_{2} X_{0}^{2} \epsilon_{1}\left(a / a_{1}\right)^{-3}$ in the regime where $X$ is close to $X_{0}$, the field equation of state is given by

$$
w_{\phi} \simeq-\left[1-\frac{4 P_{2}}{P_{0}} X_{0}^{2} \epsilon_{1}\left(\frac{a}{a_{1}}\right)^{-3}\right]^{-1}
$$

Since $w_{\phi} \rightarrow-1$ at late times it is possible to give rise to the cosmic acceleration. One can also realize $w_{\phi} \approx 0$ during the matter era, provided that the condition $4 P_{2} X_{0}^{2} /\left|P_{0}\right| \gg 1$ is satisfied. The sound speed squared defined in Eq. 67] is approximately given by

$$
c_{s}^{2} \simeq \frac{1}{2} \epsilon_{1}\left(\frac{a}{a_{1}}\right)^{-3},
$$

which is much smaller than unity. Hence the large sound speed problem can be evaded in the model (112). In Ref. [383] it was shown that the above purely k-essence model is equivalent to a fluid with a closed-form barotropic equation of state plus a constant term that works as a cosmological constant to all orders in structure formation. See Refs. 384 388] for generalized versions of the above model.

\section{MODIFIED GRAVITY MODELS}

There is another class of dark energy models in which gravity is modified from General Relativity (GR). We review a number of cosmological and gravitational aspects of $f(R)$ gravity, Gauss-Bonnet gravity, scalar-tensor theories, and a braneworld model. We also discuss observational signatures of those models to distinguish them from other dark energy models.

\section{A. $f(R)$ gravity}

The simplest modification to GR is $f(R)$ gravity with the action

$$
S=\frac{1}{2 \kappa^{2}} \int \mathrm{d}^{4} x \sqrt{-g} f(R)+\int \mathrm{d}^{4} x \mathcal{L}_{M}\left(g_{\mu \nu}, \Psi_{M}\right),
$$

where $f$ is a function of the Ricci scalar $R$ and $\mathcal{L}_{M}$ is a matter Lagrangian for perfect fluids. The Lagrangian $\mathcal{L}_{M}$ depends on the metric $g_{\mu \nu}$ and the matter fields $\Psi_{M}$. We do not consider a direct coupling between the Ricci scalar and matter (such as $f_{1}(R) \mathcal{L}_{M}$ studied in Refs. 389 391]).

\section{Viable $f(R)$ dark energy models}

In the standard variational approach called the metric formalism, the affine connections $\Gamma_{\mu \nu}^{\lambda}$ are related with the metric $g_{\mu \nu}$ [67]. In this formalism the field equation can be derived by varying the action (116) with respect to $g_{\mu \nu}$ :

$$
F(R) R_{\mu \nu}(g)-\frac{1}{2} f(R) g_{\mu \nu}-\nabla_{\mu} \nabla_{\nu} F(R)+g_{\mu \nu} \square F(R)=\kappa^{2} T_{\mu \nu}
$$

where $F(R) \equiv \partial f / \partial R$, and $T_{\mu \nu}=-(2 / \sqrt{-g}) \delta \mathcal{L}_{M} / \delta g^{\mu \nu}$ is the energy-momentum tensor of matter. Note that there is another way for the variation of the action called the Palatini formalism in which the metric and the connections are treated as independent variables. In Sec. VA4 we shall briefly mention the application of Palatini $f(R)$ gravity to dark energy. The trace of Eq. (117) is given by

$$
3 \square F(R)+F(R) R-2 f(R)=\kappa^{2} T,
$$

where $T=g^{\mu \nu} T_{\mu \nu}=-\rho_{M}+3 P_{M}$. Here $\rho_{M}$ and $P_{M}$ are the energy density and the pressure of matter, respectively. 
The de Sitter point corresponds to a vacuum solution at which the Ricci scalar is constant. Since $\square F(R)=0$ at this point, we obtain

$$
F(R) R-2 f(R)=0 \text {. }
$$

The model $f(R)=\alpha R^{2}$ satisfies this condition and hence it gives rise to an exact de Sitter solution. In fact the first model of inflation proposed by Starobinsky [113] corresponds to $f(R)=R+\alpha R^{2}$, in which the cosmic acceleration ends when the term $\alpha R^{2}$ becomes smaller than $R$. Dark energy models based on $f(R)$ theories can be also constructed to realize the late-time de Sitter solution satisfying the condition (119).

The possibility of the late-time cosmic acceleration in $f(R)$ gravity was first suggested by Capozziello [47] in 2002. An $f(R)$ dark energy model of the form $f(R)=R-\mu^{2(n+1)} / R^{n}(n>0)$ was proposed in Refs. [49 52] (see also Refs. [392 397]), but it became clear that this model suffers from a number of problems such as the matter instability [398], absence of the matter era [399, 400], and inability to satisfy local gravity constraints [401 406]. This problem arises from the fact that $f_{, R R}<0$ in this model.

In order to see why the models with negative values of $f_{, R R}$ are excluded, let us consider local fluctuations on a background characterized by a curvature $R_{0}$ and a density $\rho_{0}$. We expand Eq. (118) in powers of fluctuations under a weak field approximation. We decompose the quantities $F(R), g_{\mu \nu}$, and $T_{\mu \nu}$ into the background part and the perturbed part: $R=R^{(0)}+\delta R, F=F^{(0)}\left(1+\delta_{F}\right), g_{\mu \nu}=\eta_{\mu \nu}+h_{\mu \nu}$, and $T_{\mu \nu}=T_{\mu \nu}^{(0)}+\delta T_{\mu \nu}$, where we have used the approximation that $g_{\mu \nu}^{(0)}$ corresponds the metric $\eta_{\mu \nu}$ in the Minkowski space-time. Then the trace equation (118) reads [403, 404]

$$
\left(\frac{\partial^{2}}{\partial t^{2}}-\nabla^{2}\right) \delta_{F}+M^{2} \delta_{F}=-\frac{\kappa^{2}}{3 F^{(0)}} \delta T,
$$

where $\delta T \equiv \eta^{\mu \nu} \delta T_{\mu \nu}$, and

$$
M^{2} \equiv \frac{1}{3}\left[\frac{f_{, R}\left(R^{(0)}\right)}{f_{, R R}\left(R^{(0)}\right)}-R^{(0)}\right]=\frac{R^{(0)}}{3}\left[\frac{1}{m\left(R^{(0)}\right)}-1\right] .
$$

Here the quantity $m=R f_{, R R} / f_{, R}$ characterizes the deviation from the $\Lambda$ CDM model $(f(R)=R-2 \Lambda)$. In the homogeneous and isotropic cosmological background (without a Hubble friction), $\delta_{F}$ is a function of the cosmic time $t$ only and Eq. (120) reduces to

$$
\ddot{\delta_{F}}+M^{2} \delta_{F}=\frac{\kappa^{2}}{3 F^{(0)}} \rho,
$$

where $\rho \equiv-\delta T$. For the models where the deviation from the $\Lambda$ CDM model is small, we have $m\left(R^{(0)}\right) \ll 1$ so that $\left|M^{2}\right|$ is much larger than $R^{(0)}$. If $M^{2}<0$, the perturbation $\delta_{F}$ exhibits a violent instability. Then the condition $M^{2} \simeq f_{, R}\left(R^{(0)}\right) /\left(3 f_{, R R}\left(R^{(0)}\right)\right)>0$ is needed for the stability of cosmological perturbations. We also require that $f_{, R}\left(R^{(0)}\right)>0$ to avoid anti-gravity (i.e. to avoid that the graviton becomes a ghost). Hence the condition $f_{, R R}\left(R^{(0)}\right)>0$ needs to hold for avoiding a tachyonic instability associated with the negative mass squared [407 411].

For the consistency with local gravity constraints in solar system, the function $f(R)$ needs to be close to that in the $\Lambda \mathrm{CDM}$ model in the region of high density (in the region where the Ricci scalar $R$ is much larger than the cosmological Ricci scalar $R_{0}$ today). We also require the existence of a stable late-time de Sitter point given in Eq. (119). From the stability analysis about the de Sitter point, one can show that it is stable for $0<m=R f_{, R R} / f_{, R}<1[412[414$ ]. Then we can summarize the conditions for the viability of $f(R)$ dark energy models:

- (i) $f_{, R}>0$ for $R \geq R_{0}$.

- (ii) $f_{, R R}>0$ for $R \geq R_{0}$.

- (iii) $f(R) \rightarrow R-2 \Lambda$ for $R \gg R_{0}$.

- (iv) $0<R f_{, R R} / f_{, R}<1$ at the de Sitter point satisfying $R f_{, R}=2 f$.

The examples of viable models satisfying all these requirements are 415 417]

$$
\begin{aligned}
& \text { (A) } f(R)=R-\mu R_{c} \frac{\left(R / R_{c}\right)^{2 n}}{\left(R / R_{c}\right)^{2 n}+1} \quad \text { with } n, \mu, R_{c}>0, \\
& \begin{array}{l}
\text { (B) } f(R)=R-\mu R_{c}\left[1-\left(1+R^{2} / R_{c}^{2}\right)^{-n}\right] \quad \text { with } n, \mu, R_{c}>0, \\
\text { (C) } f(R)=R-\mu R_{c} \tanh \left(R / R_{c}\right) \quad \text { with } \mu, R_{c}>0,
\end{array}
\end{aligned}
$$


where $\mu, R_{c}$, and $n$ are constants. Models similar to $(\mathrm{C})$ were proposed in Refs. [418, 419]. Note that $R_{c}$ is roughly of the order of the present cosmological Ricci scalar $R_{0}$. If $R \gg R_{c}$ the models are close to the $\Lambda$ CDM model $\left(f(R) \simeq R-\mu R_{c}\right)$, so that $\mathrm{GR}$ is recovered in the region of high density. The models (A) and (B) have the following asymptotic behavior

$$
f(R) \simeq R-\mu R_{c}\left[1-\left(R / R_{c}\right)^{-2 n}\right], \quad\left(R \gg R_{c}\right)
$$

which rapidly approaches the $\Lambda$ CDM model for $n \gtrsim 1$. The model (C) shows an even faster decrease of $m$ in the region $R \gg R_{c}$. The model $f(R)=R-\mu R_{c}\left(R / R_{c}\right)^{n}(0<n<1)$ proposed in Refs. [414, 420] is also viable, but it does not allow the rapid decrease of $m$ in the region of high density required for the consistency with local gravity tests.

For example, let us consider the model (B). The de Sitter point given by the condition (119) satisfies

$$
\mu=\frac{x_{1}\left(1+x_{1}^{2}\right)^{n+1}}{2\left[\left(1+x_{1}^{2}\right)^{n+1}-1-(n+1) x_{1}^{2}\right]},
$$

where $x_{1} \equiv R_{1} / R_{c}$ and $R_{1}$ is the Ricci scalar at the de Sitter point. The stability condition $(0<m<1)$ at this point gives [416]

$$
\left(1+x_{1}^{2}\right)^{n+2}>1+(n+2) x_{1}^{2}+(n+1)(2 n+1) x_{1}^{4}
$$

The condition (128) gives the lower bound on the parameter $\mu$. When $n=1$ one has $x_{1}>\sqrt{3}$ and $\mu>8 \sqrt{3} / 9$. Under Eq. (128) one can show that the conditions $f_{, R}>0$ and $f_{, R R}>0$ are also satisfied for $R \geq R_{1}$.

\section{Observational signatures of $f(R)$ dark energy models}

In the flat FLRW space-time we obtain the following equations of motion from Eqs. (117) and (118):

$$
\begin{aligned}
3 F H^{2} & =\kappa^{2} \rho_{m}+(F R-f) / 2-3 H \dot{F}, \\
2 F \dot{H} & =-\kappa^{2} \rho_{m}-\ddot{F}+H \dot{F},
\end{aligned}
$$

where, for the perfect fluid, we have taken into account only the non-relativistic matter with energy density $\rho_{m}$. In order to confront $f(R)$ dark energy models with SN Ia observations, we rewrite Eqs. (129) and (130) as follows:

$$
\begin{aligned}
& 3 A H^{2}=\kappa^{2}\left(\rho_{m}+\rho_{\mathrm{DE}}\right), \\
& -2 A \dot{H}=\kappa^{2}\left(\rho_{m}+\rho_{\mathrm{DE}}+P_{\mathrm{DE}}\right),
\end{aligned}
$$

where $A$ is some constant and

$$
\begin{aligned}
\kappa^{2} \rho_{\mathrm{DE}} & \equiv(1 / 2)(F R-f)-3 H \dot{F}+3 H^{2}(A-F) \\
\kappa^{2} P_{\mathrm{DE}} & \equiv \ddot{F}+2 H \dot{F}-(1 / 2)(F R-f)-\left(3 H^{2}+2 \dot{H}\right)(A-F) .
\end{aligned}
$$

By defining $\rho_{\mathrm{DE}}$ and $P_{\mathrm{DE}}$ in this way, one can easily show that the following continuity equation holds

$$
\dot{\rho}_{\mathrm{DE}}+3 H\left(\rho_{\mathrm{DE}}+P_{\mathrm{DE}}\right)=0
$$

We define the dark energy equation of state $w_{\mathrm{DE}} \equiv P_{\mathrm{DE}} / \rho_{\mathrm{DE}}$, which is directly related to the one used in SN Ia observations. From Eqs. (131) and (132) it is given by [83, 421]

$$
w_{\mathrm{DE}}=-\frac{2 A \dot{H}+3 A H^{2}}{3 A H^{2}-\kappa^{2} \rho_{m}}=\frac{w_{\mathrm{eff}}}{1-(F / A) \tilde{\Omega}_{m}},
$$

where $\tilde{\Omega}_{m} \equiv \kappa^{2} \rho_{m} /\left(3 F H^{2}\right)$. The viable $f(R)$ models approach the $\Lambda$ CDM model in the past, i.e. $F \rightarrow 1$ as $R \rightarrow \infty$. In order to reproduce the standard matter era in the high-redshift regime we can choose $A=1$ in Eqs. (131) and (132). Another possible choice is $A=F_{0}$, where $F_{0}$ is the present value of $F$. This choice is suitable if the deviation of $F_{0}$ from 1 is small (as in scalar-tensor theory with a massless scalar field 422,423 ). In both cases the equation of state $w_{\mathrm{DE}}$ can be smaller than -1 before reaching the de Sitter attractor [83, 415, 417, 419, 424]. This originates from the fact that the presence of non-relativistic matter makes the denominator in Eq. (136) smaller than 1 (unlike 


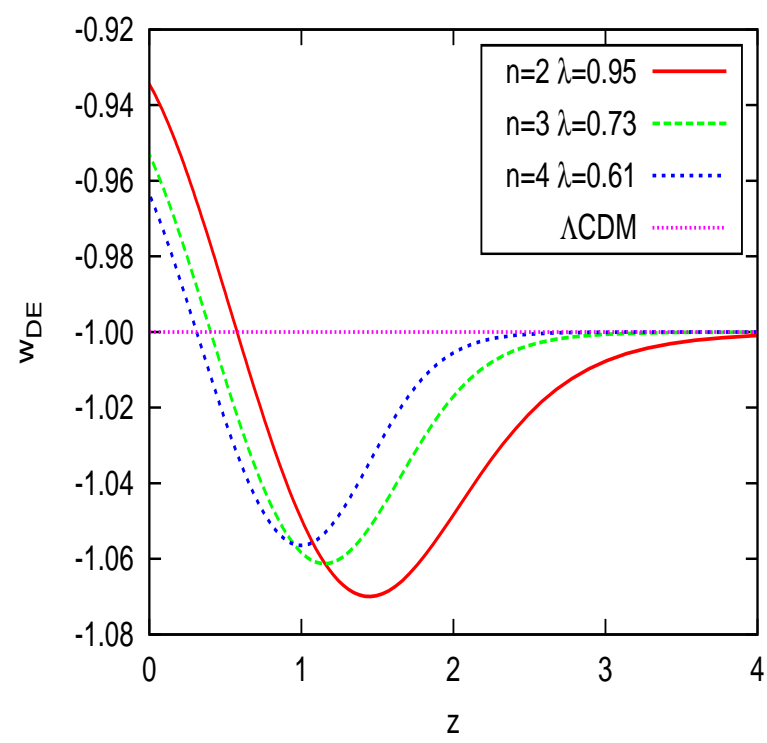

Figure 5: Evolution of the dark energy equation of state $w_{\mathrm{DE}}$ for the model (B) with $A=1$ in Eqs. (131) and (132). The phantom equation of state and the cosmological constant boundary crossing are realized. From Ref. [424].

Refs. [425, 426] in which the authors did not take into account the contribution of non-relativistic matter). Thus $f(R)$ dark energy models give rise to a phantom equation of state without violating stability conditions of the system. The models (A) and (B) are allowed from the SN Ia observations provided that $n$ is larger than the order of unity [427 430].

The modification of gravity manifests itself in the effective gravitational coupling that appears in the equation of cosmological perturbations. The full perturbation equations in $f(R)$ gravity are presented in Refs. [431 433]. When we confront $f(R)$ models with the observations of large-scale structure, the wavenumbers $k$ of interest are sub-horizon modes with $k / a \gg H$. We can employ a so-called quasi-static approximation under which the dominant terms in perturbation equations correspond to those including $k^{2} / a^{2}, \delta \rho_{m}$, and $M^{2}$ [9, 91, 423, 434]. Then the matter density perturbation $\delta_{m}$ approximately satisfies the following equation [434, 435]

$$
\ddot{\delta}_{m}+2 H \dot{\delta}_{m}-4 \pi G_{\text {eff }} \rho_{m} \delta_{m} \simeq 0,
$$

where $\rho_{m}$ is the energy density of non-relativistic matter, and

$$
G_{\mathrm{eff}}=\frac{G}{f_{, R}} \frac{1+4 m k^{2} /\left(a^{2} R\right)}{1+3 m k^{2} /\left(a^{2} R\right)}
$$

where $m \equiv R f_{, R R} / f_{, R}$. This approximation is accurate for viable $f(R)$ dark energy models as long as an oscillating mode of the scalar-field degree of freedom is suppressed relative to the matter-induced mode [416, 417, 436 439].

In the regime where the deviation from the $\Lambda \mathrm{CDM}$ model is small such that $m k^{2} /\left(a^{2} R\right) \ll 1$, the effective gravitational coupling $G_{\text {eff }}$ is very close to the gravitational constant $G$. Then the matter perturbation evolves as $\delta_{m} \propto t^{2 / 3}$ during the matter dominance. Meanwhile in the regime $m k^{2} /\left(a^{2} R\right) \gg 1$ one has $G_{\text {eff }} \simeq 4 G /\left(3 f_{, R}\right)$, so that the evolution of $\delta_{m}$ during the matter era is given by $\delta_{m} \propto t^{(\sqrt{33}-1) / 6}$ [416, 417]. The transition from the former regime to the latter regime occurs at the critical redshift [440]

$$
z_{k} \simeq\left[\left(\frac{k}{a_{0} H_{0}}\right)^{2} \frac{2 n(2 n+1)}{\mu^{2 n}} \frac{\left(2\left(1-\Omega_{m}^{(0)}\right)\right)^{2 n+1}}{\left(\Omega_{m}^{(0)}\right)^{2(n+1)}}\right]^{1 /(6 n+4)}-1,
$$

where " 0 " represents the values today. The time $t_{k}$ at the transition has a scale-dependence $t_{k} \propto k^{-3 /(6 n+4)}$, which means that the transition occurs earlier for larger $k$. The matter power spectrum $P_{\delta_{m}}=\left|\delta_{m}\right|^{2}$ at the onset of cosmic acceleration (at time $t_{\Lambda}$ ) shows a difference compared to the case of the $\Lambda$ CDM model [416]:

$$
\frac{P_{\delta_{m}}}{P_{\delta_{m}} \Lambda \mathrm{CDM}}=\left(\frac{t_{\Lambda}}{t_{k}}\right)^{2\left(\frac{\sqrt{33}-1}{6}-\frac{2}{3}\right)} \propto k^{\frac{\sqrt{33}-5}{6 n+4}} .
$$


The ratio of the two power spectra today, i.e. $P_{\delta_{m}}\left(t_{0}\right) / P_{\delta_{m}}{ }^{\Lambda \mathrm{CDM}}\left(t_{0}\right)$ is in general different from Eq. (140), but the difference is small for $n$ of the order of unity [417].

The modified evolution of perturbations for the redshift $z<z_{k}$ gives rise to the integrated Sachs-Wolfe (ISW) effect in CMB anisotropies [420, 441 443], but this is limited to very large scales (low multipoles). Since the CMB spectrum on the scales relevant to the large-scale structure $\left(k \gtrsim 0.01 h \mathrm{Mpc}^{-1}\right)$ is hardly affected by this modification, there is a difference between the spectral indices of the CMB spectrum and the galaxy power spectrum: $\Delta n_{s}=$ $(\sqrt{33}-5) /(6 n+4)$. Observationally we do not find any strong signature for the difference of slopes of the two spectra. If we take the mild bound $\Delta n_{s}<0.05$, we obtain the constraint $n>2$.

The growth index $\gamma$ defined in Eq. (78) can be as close as 0.4 today in viable $f(R)$ models given in Eqs. (123)-(125) 421]. Depending on the epoch at which the perturbations cross the transition redshift $z_{k}$, the spatial dispersion of the growth index $\gamma_{0} \equiv \gamma(z=0)$ appears in the range of values $0.40<\gamma<0.55$. There are also regions in parameter space for which $\gamma_{0}$ converges to values around $0.40<\gamma<0.43$ [440]. These unusual dispersed or converged spectra will be useful to distinguish between $f(R)$ gravity models and the $\Lambda$ CDM model in future high-precision observations. Since the modified evolution of matter perturbations directly affects the shear power spectrum in weak lensing, this is another important test for probing $f(R)$ gravity observationally [444 448]. We also note that the nonlinear evolution of matter perturbations in $f(R)$ gravity (corresponding to the scales $k \gtrsim 0.1 h \mathrm{Mpc}^{-1}$ ) has been studied in Refs. [449 457].

\section{Local gravity constraints}

Let us discuss local gravity constraints on $f(R)$ dark energy models. In the region of high density where gravitational experiments are carried out, the linear expansion of $R$ in terms of the cosmological value $R^{(0)}$ and the perturbation $\delta R$ is no longer valid because of the violation of the condition $\delta R \ll R^{(0)}$. In such a nonlinear regime, the chameleon mechanism [350, 351] can be at work to suppress the effective coupling between dark energy and non-relativistic matter. In order to study how the chameleon mechanism works in $f(R)$ gravity, we transform the action (116) to the Einstein frame action under the conformal transformation $\tilde{g}_{\mu \nu}=F g_{\mu \nu}$ : 458

$$
S_{E}=\int \mathrm{d}^{4} x \sqrt{-\tilde{g}}\left[\frac{1}{2 \kappa^{2}} \tilde{R}-\frac{1}{2} \tilde{g}^{\mu \nu} \partial_{\mu} \phi \partial_{\nu} \phi-V(\phi)\right]+\int \mathrm{d}^{4} x \mathcal{L}_{M}\left(g_{\mu \nu}, \Psi_{m}\right),
$$

where $\kappa \phi \equiv \sqrt{3 / 2} \ln F, V(\phi)=(R F-f) /\left(2 \kappa^{2} F^{2}\right)$, and a tilde represents quantities in the Einstein frame.

The action (141) is the same as (90) with the correspondence that $g_{\mu \nu}$ in the Jordan frame is equivalent to $g_{\mu \nu}^{(i)}$ in the action (90). Since the quantity $F$ is given by $F=e^{-2 \kappa Q \phi}$ with $Q=-1 / \sqrt{6}$ in metric $f(R)$ gravity, the field $\phi$ is coupled to non-relativistic matter (including baryons and dark matter) with a universal coupling $Q=-1 / \sqrt{6}$. Let us consider the models (123) and (124), which behave as Eq. (126) in the region of high density $\left(R \gg R_{c}\right)$. For the functional form (126) the effective potential defined in Eq. (93) is

$$
V_{\mathrm{eff}}(\phi) \simeq \frac{\mu R_{c}}{2 \kappa^{2}} e^{-4 \kappa \phi / \sqrt{6}}\left[1-(2 n+1)\left(\frac{-\kappa \phi}{\sqrt{6} n \mu}\right)^{2 n /(2 n+1)}\right]+\rho e^{-\kappa \phi / \sqrt{6}},
$$

where

$$
F=e^{2 \kappa \phi / \sqrt{6}}=1-2 n \mu\left(R / R_{c}\right)^{-(2 n+1)}
$$

Inside and outside a spherically symmetric body, the effective potential (142) has the following minima given, respectively, by

$$
\kappa \phi_{A} \simeq-\sqrt{6} n \mu\left(\frac{R_{c}}{\kappa^{2} \rho_{A}}\right)^{2 n+1}, \quad \kappa \phi_{B} \simeq-\sqrt{6} n \mu\left(\frac{R_{c}}{\kappa^{2} \rho_{B}}\right)^{2 n+1} .
$$

One has $\left|\phi_{B}\right| \gg\left|\phi_{A}\right|$ provided that $\rho_{A} \gg \rho_{B}$.

The bound (100) translates into

$$
\frac{n \mu}{x_{1}^{2 n+1}}\left(\frac{R_{1}}{\rho_{B}}\right)^{2 n+1}<1.5 \times 10^{-15}
$$

where $x_{1}=R_{1} / R_{c}$ and $R_{1}$ is the Ricci scalar at the de Sitter point. Let us consider the case in which the Lagrangian density is given by (126) for $R \geq R_{1}$. In the original models of Hu and Sawicki [415] and Starobinsky [416] there 
are some modification to the estimation of $R_{1}$, but this change is not significant when we place constraints on model parameters. The de Sitter point for the model (126) corresponds to $\mu=x_{1}^{2 n+1} /\left[2\left(x_{1}^{2 n}-n-1\right)\right]$. Substituting this relation into Eq. (145), we find

$$
\frac{n}{2\left(x_{1}^{2 n}-n-1\right)}\left(\frac{R_{1}}{\rho_{B}}\right)^{2 n+1}<1.5 \times 10^{-15} .
$$

The stability of the de Sitter point requires that $m\left(R_{1}\right)<1$, which translates into the condition $x_{1}^{2 n}>2 n^{2}+3 n+1$. Then the term $n /\left[2\left(x_{1}^{2 n}-n-1\right)\right]$ is smaller than 0.25 for $n>0$. Using the approximation that $R_{1}$ and $\rho_{B}$ are of the orders of the present cosmological density $10^{-29} \mathrm{~g} / \mathrm{cm}^{3}$ and the baryonic/dark matter density $10^{-24} \mathrm{~g} / \mathrm{cm}^{3}$ in our galaxy, respectively, we obtain the following constraint from (146): [459]

$$
n>0.9 .
$$

Thus $n$ does not need to be much larger than unity. Under the condition (147), the deviation from the $\Lambda$ CDM becomes important as $R$ decreases to the order of $R_{c}$.

From (143) we find that there is a curvature singularity with $R \rightarrow \infty$ (and $M^{2} \rightarrow \infty$ ) at $\phi=0$ for the models (123) and (124). At this singularity the field potential is finite, while its derivative goes to infinity. This singularity can be accessible as we go back to the past [460], unless the oscillating mode of the scalar-field degree of freedom is suppressed. This amounts to the fine-tuning of initial conditions for the field perturbation [416]. This past singularity can be cured by taking into account the $R^{2}$ term [461]. The model of the type $f(R)=R-\alpha R_{c} \ln \left(1+R / R_{c}\right)$ was also proposed to address this problem [462], but it satisfies neither local gravity constraints [463] nor observational constraints of large-scale structure [464]. Frolov [460] anticipated that the curvature singularity may be accessed in a strong gravitational background such as neutron stars. Kobayashi and Maeda [465, 466] showed the difficulty of obtaining static spherically symmetric solutions because of the presence of the singularity. On the other hand, the choice of accurate boundary conditions confirms the existence of static solutions in a strong gravitational background with $\Phi_{c} \lesssim 0.3[467-470]$.

\section{Palatini $f(R)$ gravity}

In the so-called Palatini formalism of $f(R)$ gravity, the connections $\Gamma_{\mu \nu}^{\lambda}$ are treated as independent variables when we vary the action (116) [471 477]. Variation of the action (116) with respect to $g_{\mu \nu}$ gives

$$
F(R) R_{\mu \nu}(\Gamma)-\frac{1}{2} f(R) g_{\mu \nu}=\kappa^{2} T_{\mu \nu},
$$

where $F(R)=\partial f / \partial R, R_{\mu \nu}(\Gamma)$ is the Ricci tensor corresponding to the connections $\Gamma_{\mu \nu}^{\lambda}$, and $T_{\mu \nu}$ is the energymomentum tensor of matter ${ }^{4} . R_{\mu \nu}(\Gamma)$ is in general different from the Ricci tensor calculated in terms of metric connections $R_{\mu \nu}(g)$. Taking the trace of Eq. (148), we find

$$
F(R) R-2 f(R)=\kappa^{2} T,
$$

where $T=g^{\mu \nu} T_{\mu \nu}$. The trace $T$ directly determines the Ricci scalar $R(T)$, which is related with the Ricci scalar $R(g)=g^{\mu \nu} R_{\mu \nu}(g)$ in the metric formalism via [482]

$$
R(T)=R(g)+\frac{3}{2\left(f^{\prime}(R(T))\right)^{2}}\left(\nabla_{\mu} f^{\prime}(R(T))\right)\left(\nabla^{\mu} f^{\prime}(R(T))\right)+\frac{3}{f^{\prime}(R(T))} \square f^{\prime}(R(T)),
$$

where a prime represents a derivative in terms of $R(T)$. Variation of the action (116) with respect to the connection leads to the following equation

$$
\begin{aligned}
R_{\mu \nu}(g)-\frac{1}{2} g_{\mu \nu} R(g)= & \frac{\kappa^{2} T_{\mu \nu}}{F}-\frac{F R(T)-f}{2 F} g_{\mu \nu}+\frac{1}{F}\left(\nabla_{\mu} \nabla_{\nu} F-g_{\mu \nu} \square F\right) \\
& -\frac{3}{2 F^{2}}\left[\partial_{\mu} F \partial_{\nu} F-\frac{1}{2} g_{\mu \nu}(\nabla F)^{2}\right] .
\end{aligned}
$$

\footnotetext{
4 There is another way for the variation of the action, known as the metric-affine formalism [478], in which the matter Lagrangian $\mathcal{L}_{M}$ depends not only on the metric $g_{\mu \nu}$ but also on the connection $\Gamma_{\mu \nu}^{\lambda}$. See Refs. [479 [481] for the detail of such an approach.
} 
Unlike the trace equation (118) in the metric formalism, the kinetic term $\square F$ is not present in the corresponding equation (149) in the Palatini formalism. Since the time-derivatives of the scalar-field degree of freedom do not appear in Palatini $f(R)$ gravity, cosmological solutions are not plagued by the dominance of the oscillating mode in the past. In fact, the sequence of radiation, matter, and de Sitter epochs can be realized even for the model $f(R)=R-\alpha / R^{n}$ $(n>0)$ [483 489]. The combined data analysis of SN Ia, BAO, and the CMB shift parameter places the bound $n \in[-0.23,0.42]$ on the model $f(R)=R-\alpha / R^{n}(n>-1)$ [489].

Although the background cosmology is well behaved in Palatini $f(R)$ gravity, the evolution of non-relativistic matter perturbations exhibits a distinguished feature relative to that in the $\Lambda$ CDM model [435, 490 492]. Under the quasi-static approximation on sub-horizon scales, the equation of matter perturbations is given by [435]

$$
\ddot{\delta}_{m}+2 H \dot{\delta}_{m}-\frac{\kappa^{2} \rho_{m}}{2 F}\left[1+\frac{m k^{2} /\left(a^{2} R\right)}{1-m}\right] \delta_{m} \simeq 0 .
$$

Although the matter perturbation evolves as $\delta_{m} \propto t^{2 / 3}$ in the regime $|m| k^{2} /\left(a^{2} R\right) \ll 1$, the evolution of $\delta_{m}$ in the regime $|m| k^{2} /\left(a^{2} R\right) \gg 1$ is completely different from that in GR. After the perturbations enter the regime $|m| k^{2} /\left(a^{2} R\right) \gtrsim 1$, they exhibit violent growth or damped oscillations depending on the signs of $m$ [435]. The $f(R)$ models are consistent with observations of large-scale structure if the perturbations do not enter the regime $|m| k^{2} /\left(a^{2} R\right) \gtrsim 1$ by today. This translates into the condition

$$
|m(z=0)| \lesssim\left(a_{0} H_{0} / k\right)^{2} .
$$

If we take the maximum wavenumber $k \approx 0.2 \mathrm{~h} \mathrm{Mpc}^{-1}$ (i.e., $k \approx 600 a_{0} H_{0}$ ), Eq. (153) gives the bound $|m(z=0)| \lesssim 3 \times$ $10^{-6}$. Hence the $f(R)$ models in the Palatini formalism are hardly distinguishable from the $\Lambda$ CDM model.

There are also a number of problems in Palatini $f(R)$ dark energy models associated with the non-dynamical nature of the scalar-field degree of freedom. The dark energy model $f(R)=R-\mu^{4} / R$ is in conflict with the Standard Model of particle physics [475 477, 493 497] because of large non-perturbative corrections to the matter Lagrangian. If we consider the models $f(R)=R-\mu^{2(n+1)} / R^{n}$, the only way to make such corrections small is to choose $n$ very close to 0 [433]. Hence the deviation from the $\Lambda$ CDM model needs to be very small. It was also shown that, for $f(R)$ dark energy models, a divergent behavior arises for the Ricci scalar at the surface of a static spherically symmetric star with a polytropic equation of state $P=c \rho_{0}^{\Gamma}(3 / 2<\Gamma<2)$, where $P$ is the pressure and $\rho_{0}$ is the rest-mass density [497, 498]. These results show that Palatini $f(R)$ dark energy models are difficult to be compatible with observational and experimental constraints, although this may not be the case for $f(R)$ models close to the Planck scale [499 501].

\section{B. Gauss-Bonnet dark energy models}

It is possible to extend $f(R)$ gravity to more general theories in which the Lagrangian density $f$ is an arbitrary function of $R, P \equiv R_{\mu \nu} R^{\mu \nu}$, and $Q \equiv R_{\mu \nu \alpha \beta} R^{\mu \nu \alpha \beta}$, where $R_{\mu \nu}$ and $R_{\mu \nu \alpha \beta}$ are Ricci tensor and Riemann tensor respectively [502, 503]. The appearance of spurious spin-2 ghosts can be avoided by taking a Gauss-Bonnet (GB) combination [504 507]

$$
\mathcal{G}=R^{2}-4 R_{\mu \nu} R^{\mu \nu}+R_{\mu \nu \alpha \beta} R^{\mu \nu \alpha \beta} .
$$

A simple dark energy model motivated from low-energy effective string theory [242] is given by [508]

$$
S=\int \mathrm{d}^{4} x \sqrt{-g}\left[\frac{1}{2 \kappa^{2}} R-\frac{1}{2} g^{\mu \nu} \partial_{\mu} \phi \partial_{\nu} \phi-V(\phi)-f(\phi) \mathcal{G}\right]+S_{M},
$$

where $V(\phi)$ and $f(\phi)$ are functions of a scalar field $\phi$, and $S_{M}$ is a matter action. The coupling $f(\phi) \mathcal{G}$ allows the presence of a de Sitter solution even for a runaway field potential $V(\phi)$ [509 512]. For the exponential potential $V(\phi)=V_{0} e^{-\kappa \lambda \phi}$ and the coupling $f(\phi)=\left(f_{0} / \mu\right) e^{\mu \kappa \phi}$, it was shown in Refs. [509, 510] that a scaling matter era can be followed by the late-time de Sitter solution for $\mu>\lambda$.

Koivisto and Mota [509] found that the parameter $\lambda$ in this model is constrained to be $3.5<\lambda<4.5(95 \%$ confidence level) from the observational data of SN Ia and WMAP 3-year. The parameter $\lambda$ is constrained to be $3.5<\lambda<4.5$ at the $95 \%$ confidence level. In the second paper [511], they showed that the model is strongly disfavored from the combined data analysis including the constraints coming from BBN, LSS, BAO, and solar system data. It was shown in Refs. [510, 513] that, when the GB term dominates the dynamics, tensor perturbations are subject to negative instabilities. Amendola et al. 514 studied local gravity constraints on the above model and showed that the energy contribution coming from the GB term needs to be strongly suppressed for the consistency 
with solar-system experiments. The above results imply that the GB term with the scalar-field coupling $f(\phi) \mathcal{G}$ can hardly be the source for dark energy.

The dark energy models based on the Lagrangian density $\mathcal{L}=R /\left(2 \kappa^{2}\right)+f(\mathcal{G})$, have been studied by a number of authors $515-524]$. In the presence of a perfect fluid with an energy density $\rho_{M}$, the Friedmann equation is given by $[515,516]$

$$
3 H^{2}=\mathcal{G} f_{, \mathcal{G}}-f-24 H^{2} f_{, \mathcal{G G}} \dot{\mathcal{G}}+\rho_{M}
$$

The Hubble parameter $H=H_{1}$ at the de Sitter point satisfies $3 H_{1}^{2}=\mathcal{G}_{1} f_{\mathcal{G}}\left(\mathcal{G}_{1}\right)-f\left(\mathcal{G}_{1}\right)$, where $\mathcal{G}_{1}=24 H_{1}^{4}$. The stability of the de Sitter point requires the condition $0<H_{1}^{6} f_{, \mathcal{G G}}\left(H_{1}\right)<1 / 384$ [520]. In order to avoid the instability of solutions during radiation and matter eras, we also need the condition $f_{, \mathcal{G G}}>0$. In Ref. [520] the authors presented a number of $f(\mathcal{G})$ models that are cosmologically viable at the background level. One of such viable models is given by

$$
f(\mathcal{G})=\lambda \frac{\mathcal{G}}{\sqrt{\mathcal{G}_{*}}} \arctan \left(\frac{\mathcal{G}}{\mathcal{G}_{*}}\right)-\alpha \lambda \sqrt{\mathcal{G}_{*}}
$$

where $\alpha, \lambda$ and $\mathcal{G}_{*}$ are constants. This model can satisfy solar system constraints for a wide range of parameter space [523].

If we consider cosmological perturbations, however, there is a Ultra-Violet (UV) instability in $f(\mathcal{G})$ models associated with a negative propagation speed squared of a scalar-field degree of freedom [516, 524]. This growth of perturbations gets stronger on smaller scales, which is difficult to be compatible with the observed galaxy spectrum unless the deviation from GR is very small. Thus $f(\mathcal{G})$ dark energy models are effectively ruled out as an alternative to the $\Lambda \mathrm{CDM}$ model.

\section{Scalar-tensor theories}

There is another class of modified gravity called scalar-tensor theories in which the Ricci scalar $R$ is coupled to a scalar field $\varphi$. One of the simplest examples is Brans-Dicke (BD) theory [291] with the action

$$
S=\int \mathrm{d}^{4} x \sqrt{-g}\left[\frac{1}{2} \varphi R-\frac{\omega_{\mathrm{BD}}}{2 \varphi}(\nabla \varphi)^{2}-U(\varphi)\right]+S_{M}\left(g_{\mu \nu}, \Psi_{M}\right),
$$

where $\omega_{\mathrm{BD}}$ is the BD parameter, $U(\varphi)$ is the field potential, and $S_{M}$ is a matter action that depends on the metric $g_{\mu \nu}$ and matter fields $\Psi_{m}$. The original BD theory [291] does not have the field potential $U(\varphi)$.

The general action for scalar-tensor theories can be written as

$$
S=\int \mathrm{d}^{4} x \sqrt{-g}\left[\frac{1}{2} f(\varphi, R)-\frac{1}{2} \omega(\varphi)(\nabla \varphi)^{2}\right]+S_{M}\left(g_{\mu \nu}, \Psi_{M}\right),
$$

where $f$ is a general function of the scalar field $\varphi$ and the Ricci scalar $R, \omega$ is a function of $\varphi$. We choose the unit $\kappa^{2}=1$. We consider theories of the type

$$
f(\varphi, R)=F(\varphi) R-2 U(\varphi) .
$$

Under the conformal transformation $\tilde{g}_{\mu \nu}=F g_{\mu \nu}$, the action in the Einstein frame is given by [458]

$$
S_{E}=\int \mathrm{d}^{4} x \sqrt{-\tilde{g}}\left[\frac{1}{2} \tilde{R}-\frac{1}{2}(\tilde{\nabla} \phi)^{2}-V(\phi)\right]+S_{M}\left(g_{\mu \nu}, \Psi_{M}\right),
$$

where $V=U / F^{2}$. We have introduced a new scalar field $\phi$ in order to make the field kinetic term canonical:

$$
\phi \equiv \int \mathrm{d} \varphi \sqrt{\frac{3}{2}\left(\frac{F_{, \varphi}}{F}\right)^{2}+\frac{\omega}{F}} .
$$

We define the coupling between dark energy and non-relativistic matter, as

$$
Q \equiv-\frac{F_{, \phi}}{2 F}=-\frac{F_{, \varphi}}{F}\left[\frac{3}{2}\left(\frac{F_{, \varphi}}{F}\right)^{2}+\frac{\omega}{F}\right]^{-1 / 2} .
$$


In $f(R)$ gravity we have $\omega=0$ and hence $F=\exp (\sqrt{2 / 3} \phi)$ from Eq. (162). Then the coupling is given by $Q=-1 / \sqrt{6}$ from Eq. (163). If $Q$ is constant as in $f(R)$ gravity, the following relations hold from Eqs. (162) and (163):

$$
F=e^{-2 Q \phi}, \quad \omega=\left(1-6 Q^{2}\right) F\left(\frac{\mathrm{d} \phi}{\mathrm{d} \varphi}\right)^{2} .
$$

In this case the action (159) in the Jordan frame reads [292]

$$
S=\int \mathrm{d}^{4} x \sqrt{-g}\left[\frac{1}{2} F(\phi) R-\frac{1}{2}\left(1-6 Q^{2}\right) F(\phi)(\nabla \phi)^{2}-U(\phi)\right]+S_{M}\left(g_{\mu \nu}, \Psi_{m}\right) .
$$

In the limit that $Q \rightarrow 0$ the action (165) reduces to the one for a minimally coupled scalar field $\phi$ with the potential $U(\phi)$. The transformation of the Jordan frame action (165) under the conformal transformation $\tilde{g}_{\mu \nu}=e^{-2 Q \phi} g_{\mu \nu}$ gives rise to the Einstein frame action (161) with a constant coupling $Q$.

One can compare (165) with the action (158) in BD theory. Setting $\varphi=F=e^{-2 Q \phi}$, one finds that two actions are equivalent if the parameter $\omega_{\mathrm{BD}}$ is related to $Q$ via the relation [292, 351]

$$
3+2 \omega_{\mathrm{BD}}=\frac{1}{2 Q^{2}} .
$$

Using this relation, we find that the General Relativistic limit $\left(\omega_{\mathrm{BD}} \rightarrow \infty\right)$ corresponds to the vanishing coupling $(Q \rightarrow 0)$. Since $Q=-1 / \sqrt{6}$ in $f(R)$ gravity, this corresponds to the Brans-Dicke parameter $\omega_{\mathrm{BD}}=0$ [401, [525, 526]. One can show that the field equation (151) in Palatini $f(R)$ gravity is equivalent to the one derived in BD theory with $\omega_{\mathrm{BD}}=-3 / 2[433,482]$. Hence Palatini $f(R)$ gravity corresponds to the infinite coupling $\left(Q^{2} \rightarrow \infty\right)$.

There are also other scalar-tensor theories that give rise to field-dependent couplings $Q(\phi)$. For a nonminimally coupled scalar field with $F(\varphi)=1-\xi \varphi^{2}$ and $\omega(\varphi)=1$ in the action (159) with (160), the coupling is field-dependent, i.e. $Q(\varphi)=\xi \varphi /\left[1-\xi \varphi^{2}(1-6 \xi)\right]^{1 / 2}$. The cosmological dynamics of dark energy models based on such theories have been studied by a number of authors [286, 527 532].

Let us consider $\mathrm{BD}$ theory with the action (165). In the absence of the potential $U(\phi)$ the BD parameter $\omega_{\mathrm{BD}}$ is constrained to be $\omega_{\mathrm{BD}}>4.0 \times 10^{4}$ from solar-system experiments [354]. This bound also applies to the case of a nearly massless field with the potential $U(\phi)$ in which the Yukawa correction $e^{-M r}$ is close to unity (where $M$ is the scalar field mass and $r$ is an interaction length). Using the bound $\omega_{\mathrm{BD}}>4.0 \times 10^{4}$ in Eq. (166), we find

$$
|Q|<2.5 \times 10^{-3} \text {. }
$$

In this case the cosmological evolution for such theories is hardly distinguishable from the $Q=0$ case. Even for scalar-tensor theories with such small couplings, it was shown that the phantom equation state of dark energy can be realized without the appearance of a ghost state [533 536$]$.

In the presence of the field potential it is possible for large coupling models $(|Q|=\mathcal{O}(1))$ to satisfy local gravity constraints under the chameleon mechanism, provided that the mass $M$ of the field $\phi$ is sufficiently large in the region of high density. In metric $f(R)$ gravity $(Q=-1 / \sqrt{6})$ the field potential $U(\phi)$ in Eq. (165) corresponds to $U=(F R-f) / 2$ with $\phi=\sqrt{3 / 2} \ln F$. The viable $f(R)$ dark energy models (123) and (124) have the asymptotic form (126), in which case the field potential is given by

$$
U(\phi)=\frac{\mu R_{c}}{2}\left[1-\frac{2 n+1}{(2 n \mu)^{2 n /(2 n+1)}}\left(1-e^{2 \phi / \sqrt{6}}\right)^{2 n /(2 n+1)}\right] .
$$

For $\mathrm{BD}$ theories with the constant coupling $Q$, one can generalize the potential (168) to the form

$$
U(\phi)=U_{0}\left[1-C\left(1-e^{-2 Q \phi}\right)^{p}\right] \quad\left(U_{0}>0, C>0,0<p<1\right) .
$$

As $\phi \rightarrow 0$, the potential (169) approaches the finite value $U_{0}$ with a divergence of the field mass squared $M^{2}=$ $U_{, \phi \phi} \rightarrow \infty$. This model has a curvature singularity at $\phi=0$ as in the case of the $f(R)$ models (123) and (124). The mass $M$ decreases as the field evolves away from $\phi=0$. The late-time cosmic acceleration can be realized by the potential (169) provided that $U_{0}$ is of the order of $H_{0}^{2}$.

Since the action (161) in the Einstein frame is equivalent to the action (90), the chameleon mechanism can be at work even for $\mathrm{BD}$ theories with large couplings $(|Q|=\mathcal{O}(1))$. Considering a spherically symmetric body with homogenous densities $\rho_{A}$ and $\rho_{B}$ inside and outside bodies respectively, the effective potential $V_{\text {eff }}=V(\phi)+e^{Q \phi} \rho$ in the Einstein frame (where $V(\phi)=U(\phi) / F^{2}$ ) has two minima characterized by

$$
\phi_{A} \simeq \frac{1}{2 Q}\left(\frac{2 U_{0} p C}{\rho_{A}}\right)^{1 /(1-p)}, \quad \phi_{B} \simeq \frac{1}{2 Q}\left(\frac{2 U_{0} p C}{\rho_{B}}\right)^{1 /(1-p)} .
$$


Using the experimental bound (100) coming from the violation of equivalence principle together with the condition for realizing the cosmic acceleration today, we obtain the constraint 292]

$$
p>1-\frac{5}{13.8-\log _{10}|Q|} .
$$

When $|Q|=10^{-2}$ and $|Q|=10^{-1}$ we have $p>0.68$ and $p>0.66$, respectively. In $f(R)$ gravity the above bound corresponds to $p>0.65$, which translates into $n>0.9$ for the model (126).

The evolution of cosmological perturbations in scalar-tensor theories has been discussed in Refs. 292, 423, 434, 537, 538]. Under the quasi-static approximation on sub-horizon scales, the matter perturbation $\delta_{m}$ for the theory (165) obeys the following equation of motion [292, 538]

$$
\ddot{\delta}_{m}+2 H \dot{\delta}_{m}-4 \pi G_{\text {eff }} \rho_{m} \delta_{m} \simeq 0
$$

where the effective (cosmological) gravitational coupling is

$$
G_{\text {eff }}=\frac{G}{F} \frac{\left(k^{2} / a^{2}\right)\left(1+2 Q^{2}\right) F+M^{2}}{\left(k^{2} / a^{2}\right) F+M^{2}} .
$$

Here $M^{2} \equiv U_{, \phi \phi}$ is the field mass squared. In the "General Relativistic" regime characterized by $M^{2} / F \gg k^{2} / a^{2}$, one has $G_{\text {eff }} \simeq G / F$ and $\delta_{m} \propto t^{2 / 3}$. In the "scalar-tensor" regime characterized by $M^{2} / F \ll k^{2} / a^{2}$, it follows that $G_{\text {eff }} \simeq\left(1+2 Q^{2}\right) G / F$ and $\delta_{m} \propto t^{\left(\sqrt{25+48 Q^{2}}-1\right) / 6}$. If the transition from the former regime to the latter regime occurs during the matter era, this gives rise to a difference between the spectral indices of the matter power spectrum and of the CMB spectrum on the scales $0.01 h \mathrm{Mpc}^{-1} \lesssim k \lesssim 0.2 h \mathrm{Mpc}^{-1}$ [292]:

$$
\Delta n_{s}=\frac{(1-p)\left(\sqrt{25+48 Q^{2}}-5\right)}{4-p} .
$$

Under the criterion $\Delta n_{s}<0.05$, we obtain the bounds $p>0.957$ for $Q=1$ and $p>0.855$ for $Q=0.5$. As long as $p$ is close to 1 , the model can be consistent with both cosmological and local gravity constraints.

For the perturbed metric $\mathrm{d} s^{2}=-(1+2 \Psi) \mathrm{d} t^{2}+a^{2}(t)(1-2 \Phi) \delta_{i j} \mathrm{~d} x^{i} \mathrm{~d} x^{j}$, the gravitational potentials obey the following equations under a quasi-static approximation on sub-horizon scales [292]

$$
\begin{aligned}
& \frac{k^{2}}{a^{2}} \Psi \simeq-\frac{4 \pi G}{F} \frac{\left(k^{2} / a^{2}\right)\left(1+2 Q^{2}\right) F+M^{2}}{\left(k^{2} / a^{2}\right) F+M^{2}} \rho_{m} \delta_{m}, \\
& \frac{k^{2}}{a^{2}} \Phi \simeq-\frac{4 \pi G}{F} \frac{\left(k^{2} / a^{2}\right)\left(1-2 Q^{2}\right) F+M^{2}}{\left(k^{2} / a^{2}\right) F+M^{2}} \rho_{m} \delta_{m},
\end{aligned}
$$

where we have recovered the gravitational constant $G$. The results (175) and (176) include those in $f(R)$ gravity by setting $Q=-1 / \sqrt{6}$. In the regime $M^{2} / F \ll k^{2} / a^{2}$ the evolution of $\Psi$ and $\Phi$ is subject to change compared to that in the GR regime characterized by $M^{2} / F \gg k^{2} / a^{2}$. In general the difference from GR may be quantified by the parameters $q$ and $\zeta$ [444]:

$$
\frac{k^{2}}{a^{2}} \Phi=-4 \pi G q \rho_{m} \delta_{m}, \quad \frac{\Phi-\Psi}{\Phi}=\zeta .
$$

In the regime $M^{2} / F \ll k^{2} / a^{2}$ of scalar-tensor theory (165) it follows that $q \simeq\left(1-2 Q^{2}\right) / F$ and $\zeta \simeq-4 Q^{2} /\left(1-2 Q^{2}\right)$.

In order to confront dark energy models with the observations of weak lensing, it may be convenient to introduce the following quantity 444 ]

$$
\Sigma \equiv q(1-\zeta / 2)
$$

From the definition (177) we find that the weak lensing potential $\psi=\Phi+\Psi$ can be expressed as

$$
\psi=-8 \pi G \frac{a^{2}}{k^{2}} \rho_{m} \delta_{m} \Sigma .
$$

In scalar-tensor theory (165) one has $\Sigma=1 / F$. The effect of modified gravity theories manifests itself in weak lensing observations in at least two ways. One is the multiplication of the term $\Sigma$ on the r.h.s. of Eq. (179). Another is the modification of the evolution of $\delta_{m}$. The latter depends on two parameters $q$ and $\zeta$, or equivalently, $\Sigma$ and $\zeta$. Thus two parameters $(\Sigma, \zeta)$ will be useful to detect signatures of modified gravity theories from future surveys of weak lensing. See Refs. [539 551] for related works about testing gravitational theories in weak lensing observations. 


\section{DGP model}

In the so-caled Dvali, Gabadadze, and Porrati (DGP) [58] braneworld it is possible to realize a "self accelerating Universe" even in the absence of dark energy. In braneworlds standard model particles are confined on a 3-dimensional (3D) brane embedded in the 5-dimensional bulk space-time with large extra dimensions. In the DGP braneworld model [58] the 3-brane is embedded in a Minkowski bulk space-time with infinitely large extra dimensions. Newton gravity can be recovered by adding a 4D Einstein-Hilbert action sourced by the brane curvature to the 5D action. Such a 4D term may be induced by quantum corrections coming from the bulk gravity and its coupling with matter on the brane. In the DGP model the standard 4D gravity is recovered for small distances, whereas the effect from the 5D gravity manifests itself for large distances. The late-time cosmic acceleration can be realized without introducing a dark energy component [552, 553] (see also Ref. [554] for a generalized version of the DGP model).

The action for the DGP model is given by

$$
S=\frac{1}{2 \kappa_{(5)}^{2}} \int \mathrm{d}^{5} X \sqrt{-\tilde{g}} \tilde{R}+\frac{1}{2 \kappa_{(4)}^{2}} \int \mathrm{d}^{4} X \sqrt{-g} R-\int \mathrm{d}^{5} X \sqrt{-\tilde{g}} \mathcal{L}_{M},
$$

where $\tilde{g}_{A B}$ is the metric in the $5 \mathrm{D}$ bulk and $g_{\mu \nu}=\partial_{\mu} X^{A} \partial_{\nu} X^{B} \tilde{g}_{A B}$ is the induced metric on the brane with $X^{A}\left(x^{c}\right)$ being the coordinates of an event on the brane labelled by $x^{c}$. The 5D and 4D (reduced) gravitational constants, $\kappa_{(5)}^{2}$ and $\kappa_{(4)}^{2}$, are related with the 5D and 4D Planck masses, $M_{(5)}$ and $M_{(4)}$, via $\kappa_{(5)}^{2}=1 / M_{(5)}^{3}$ and $\kappa_{(4)}^{2}=1 / M_{(4)}^{2}$. The first and second terms in Eq. (180) correspond to Einstein-Hilbert actions in the 5D bulk and on the brane, respectively. The matter action consists of a brane-localized matter whose action is given by $\int \mathrm{d}^{4} x \sqrt{-g}\left(\sigma+\mathcal{L}_{M}^{\text {brane }}\right)$, where $\sigma$ is the 3-brane tension and $\mathcal{L}_{M}^{\text {brane }}$ is the Lagrangian density on the brane. Since the tension is not related to the Ricci scalar $R$, it can be adjusted to be zero.

The Einstein equation in the $5 \mathrm{D}$ bulk is given by $G_{A B}^{(5)}=0$, where $G_{A B}^{(5)}$ is the $5 \mathrm{D}$ Einstein tensor. Imposing the Israel junction conditions on the brane with a $Z_{2}$ symmetry, we obtain the $4 \mathrm{D}$ Einstein equation [555]

$$
G_{\mu \nu}-\frac{1}{r_{c}}\left(K_{\mu \nu}-K g_{\mu \nu}\right)=\kappa_{(4)}^{2} T_{\mu \nu}
$$

where $K_{\mu \nu}$ is the extrinsic curvature on the brane and $T_{\mu \nu}$ is the energy-momentum tensor of localized matter. The cross-over scale $r_{c}$ is defined by $r_{c} \equiv \kappa_{(5)}^{2} /\left(2 \kappa_{(4)}^{2}\right)$. The Friedmann equation on the flat FLRW brane takes a simple form 552,553 .

$$
H^{2}-\frac{\epsilon}{r_{c}} H=\frac{\kappa_{(4)}^{2}}{3} \rho_{M},
$$

where $\epsilon= \pm 1$, and $\rho_{M}$ is the energy density of matter on the brane (with pressure $P_{M}$ ) satisfying the continuity equation

$$
\dot{\rho}_{M}+3 H\left(\rho_{M}+P_{M}\right)=0 .
$$

If $r_{c}$ is much larger than the Hubble radius $H^{-1}$, the first term in Eq. (182) dominates over the second one. In this case the standard Friedmann equation, $H^{2}=\kappa_{(4)}^{2} \rho_{M} / 3$, is recovered. Meanwhile, in the regime $r_{c}<H^{-1}$, the presence of the second term in Eq. (182) leads to a modification to the standard Friedmann equation. In the Universe dominated by non-relativistic matter $\left(\rho_{M} \propto a^{-3}\right)$, the Universe approaches a de Sitter solution for $\epsilon=+1: H \rightarrow H$ dS $=1 / r_{c}$. Hence it is possible to realize the present cosmic acceleration provided that $r_{c}$ is of the order of the present Hubble radius $H_{0}^{-1}$.

Although the DGP braneworld is an attractive model allowing a self acceleration, the joint constraints from SNLS, BAO, and CMB data shows that this model is disfavored observationally [556 561]. There is a modified version of the DGP model characterized by the Friedmann equation $H^{2}-H^{\alpha} / r_{c}^{2-\alpha}=\kappa_{(4)}^{2} \rho_{m} / 3$, where $\rho_{m}$ is the energy density of non-relativistic matter 562. In Fig. 6 we show $1 \sigma$ and $2 \sigma$ contours in the $\left(\Omega_{m}^{(0)}, \alpha\right)$ plane constrained from the joint data analysis of SN Ia, BAO, CMB, gamma ray bursts, and the linear growth factor of matter perturbations [561]. The parameter $\alpha$ is constrained to be $\alpha=0.254 \pm 0.153$ (68\% confidence level) and hence the flat DGP model $(\alpha=1)$ is incompatible with observations.

The evolution of density perturbations in the DGP model has been studied in Refs. [563 571]. Under the quasistatic approximation on sub-horizon scales, the linear matter perturbation $\delta_{m}$ (with a homogenous density $\rho_{m}$ ) obeys the following equation

$$
\ddot{\delta}_{m}+2 H \dot{\delta}_{m}-4 \pi G_{\mathrm{eff}} \rho_{m} \delta_{m}=0, \quad G_{\mathrm{eff}}=[1+1 /(3 \beta)] G,
$$




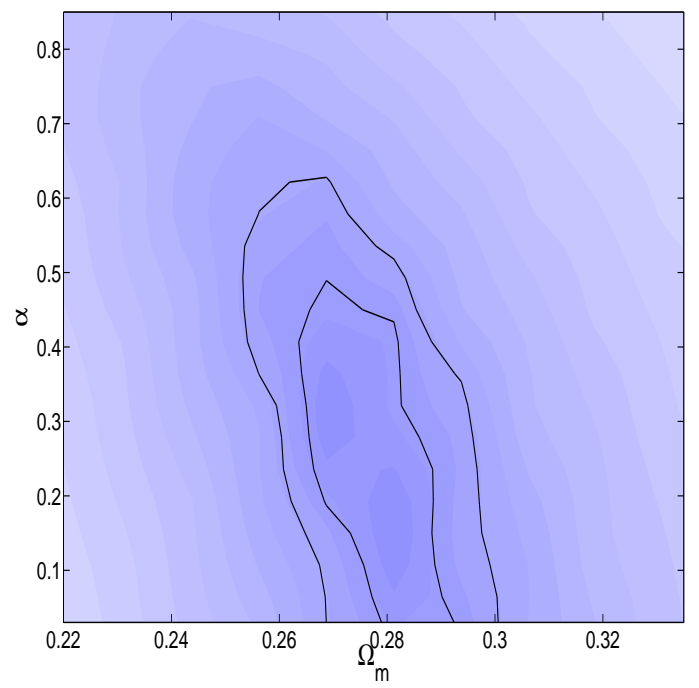

Figure 6: Combined observational constraints on the modified DGP model characterized by the Friedmann equation $H^{2}-$ $H^{\alpha} / r_{c}^{2-\alpha}=\kappa_{(4)}^{2} \rho_{m} / 3$. The two curves show $1 \sigma$ and $2 \sigma$ contours in the $\left(\Omega_{m}^{(0)}, \alpha\right)$ plane. The original DGP model $(\alpha=1)$ is incompatible with observations. From Ref. [561].

where $\beta \equiv 1-2 H r_{c}\left[1+\dot{H} /\left(3 H^{2}\right)\right]$. In the deep matter era $\left(H r_{c} \gg 1\right), \beta$ is largely negative $(|\beta| \gg 1)$. In this regime the matter perturbation evolves as $\delta_{m} \propto t^{2 / 3}$ as in GR. Around the late-time de Sitter solution one has $\beta \simeq 1-2 H r_{c} \simeq-1$ and $1+1 /(3 \beta) \simeq 2 / 3$, so that the growth rate of $\delta_{m}$ gets smaller than that in the $\Lambda$ CDM model. The growth index $\gamma$ defined in Eq. (78) is given by $\gamma \approx 0.68[323]$.

In the massless regime $\left(M^{2} / F \ll k^{2} / a^{2}\right)$ in BD theory the effective gravitational coupling (173) is given by $G_{\mathrm{eff}}=\left(1+2 Q^{2}\right) G / F=\left(4+2 \omega_{\mathrm{BD}}\right) /\left(3+2 \omega_{\mathrm{BD}}\right) G / F$, where we used the relation (166). Comparing this with the effective coupling (184), we find that the DGP model is related to BD theory via $\omega_{\mathrm{BD}}=(3 / 2)(\beta-1)$ with $F=1$. Since $\beta<0$ for the self-accelerating DGP solution, this implies that $\omega_{\mathrm{BD}}<-3 / 2$ and hence the DGP model contains a ghost mode. It is however possible to construct a generalized DGP model free from the ghost problem by embedding our visible 3 -brane with a 4 -brane in a flat $6 \mathrm{D}$ bulk [168].

In the DGP model, a brane bending mode $\phi$ in the bulk corresponds to a scalar-field degree of freedom. In general such a field can mediate a long range fifth force incompatible with local gravity experiments, but the presence of a self-interaction of $\phi$ allows the so-called Vainshtein mechanism [572] to work within a radius $r_{*}=\left(r_{g} r_{c}^{2}\right)^{1 / 3}\left(r_{g}\right.$ is the Schwarzschild radius of a source). The DGP model can be consistent with local gravity constraints under some range of conditions on the energy-momentum tensor [573 575$]$.

The DGP model stimulated other approaches for constructing ghost-free theories in the presence of nonlinear selfinteractions of a scalar field $\phi$. It is important to keep the field equations at second order in time derivatives to avoid that an extra degree of freedom gives rise to a ghost state. In particular Nicolis et al. [577] imposed a constant gradient-shift symmetry ("Galilean" symmetry), $\partial_{\mu} \phi \rightarrow \partial_{\mu} \phi+b_{\mu}$, to restrict the equations of motion at second order, while keeping a universal gravitational coupling with matter. In the 4-dimensional Minkowski space-time they found five terms $\mathcal{L}_{i}(i=1, \cdots 5)$ giving rise to equations of motion satisfying the Galilean symmetry. The first three terms are given by $\mathcal{L}_{1}=\phi, \mathcal{L}_{2}=\nabla_{\mu} \phi \nabla^{\mu} \phi$, and $\mathcal{L}_{3}=\square \phi \nabla_{\mu} \phi \nabla^{\mu} \phi$. The term $\mathcal{L}_{3}$ is the nonlinear field derivative that appears in the DGP model, which allows the possibility for the consistency with solar system experiments through the Vainshtein mechanism. Deffayet et al. [578, 579] derived the covariant expression of the terms $\mathcal{L}_{i}(i=1, \cdots 5)$ by extending the analysis to the curved space-time.

Silva and Koyama [580] considered BD theory (without a field potential) in the presence of a nonlinear derivative term $\xi(\phi) \square \phi \nabla_{\mu} \phi \nabla^{\mu} \phi$, i.e.

$$
S=\int \mathrm{d}^{4} x \sqrt{-g}\left[\frac{1}{2} \phi R-\frac{\omega_{\mathrm{BD}}}{2 \phi}(\nabla \phi)^{2}+\xi(\phi) \square \phi \nabla_{\mu} \phi \nabla^{\mu} \phi\right]+S_{M},
$$

where $S_{M}$ is the matter action. Although the nonlinear derivative term does not satisfy the Galilean invariance in the FLRW cosmological background, the field equation of motion remains at second order (see also Ref. [581]). This term also arises as one of higher-order derivative corrections to low-energy effective string theory. Interestingly, 
for the function $\xi(\phi)=1 /\left(M^{2} \phi^{2}\right)$, there exists a de Sitter solution responsible for dark energy (provided that $\left.M \approx H_{0}\right)$. Moreover, because of the presence of the nonlinear interaction, the problems of the appearance of ghosts and instabilities can be avoided for the BD parameter $\omega_{\mathrm{BD}}$ smaller than -2 [580]. At early times General Relativity can be recovered by the cosmological Vainshtein mechanism. A number of interesting observational signatures such as modified growth of matter perturbations as well as a distinguished ISW effect have been studied in Refs. 580, 582, 583].

\section{COSMIC ACCELERATION WITHOUT DARK ENERGY}

There are attempts to explain the apparent cosmic acceleration by inhomogeneities in the distribution of matter without recourse to a dark energy component (see Ref. [584] for review).

One of such approaches is the void model in which the presence of underdense bubbles leads to the faster expansion of the Universe compared to the outside. In other words we live in the middle of a huge spherical region and we interpret the evolution of this underdense region as an apparent cosmic acceleration. Originally, Tomita [59, 60] introduced a local homogenous void separated from the outside described by a homogenous FLRW space with a singular mass shell. The analysis was extended to the models with a continuous transition between the inside and outside the void [63]. This can be described by a class of the Lemaitre-Tolman-Bondi (LTB) spherically symmetric models. Theoretical and observational aspects of the LTB model have been extensively studied as an alternative to dark energy [61 63, 585,610$]$.

The second approach is based on the backreaction of cosmological perturbations arising from perturbing the homogeneous Universe [64 66]. Unlike the void model, this tries to explain a real cosmic acceleration by arranging inhomogeneities that come from the deviation of the FLRW metric.

There is another approach for explaining the apparent cosmic acceleration based on the "Ultra Strong" version of equivalence principle [611, 612]. In this model the standard geometric description of space-time as a metric manifold holds as a small distance approximation and hence General Relativity can be modified on large scales by a curvaturedependent subleading effect. Although the original model proposed in Ref. [613] do not explain the observational data of SN Ia very well, its modified version can be consistent with the SN Ia data with a rather low value of the Hubble constant, $H_{0} \approx 50 \mathrm{~km} \mathrm{sec}^{-1} \mathrm{Mpc}^{-1}$.

In the following we shall briefly review the first two approaches.

\section{A. Inhomogeneous Lemaître-Tolman-Bondi model}

In order to discuss a spherical inhomogeneity in local regions we take the LTB metric given by

$$
\mathrm{d} s^{2}=-\mathrm{d} t^{2}+X^{2}(t, r) \mathrm{d} r^{2}+R^{2}(t, r) \mathrm{d} \Omega^{2},
$$

where the expansion factor along the radial coordinate $r$ is different relative to the surface line element $\mathrm{d} \Omega^{2}=$ $\mathrm{d} \theta^{2}+\sin ^{2} \theta \mathrm{d} \phi^{2}$. Solving the $(0,1)$ component of the Einstein equation $G_{01}=0$ for the fluid at rest, it follows that $X(t, r)$ is separable as $X(t, r)=R^{\prime}(t, r) / \sqrt{1+\beta(r)}$. Here a prime represents a partial derivative with respect to $r$ and $\beta(r)$ is a function of $r$. Then the metric (186) is given by

$$
\mathrm{d} s^{2}=-\mathrm{d} t^{2}+\frac{\left[R^{\prime}(t, r)\right]^{2}}{1+\beta(r)} \mathrm{d} r^{2}+R^{2}(t, r) \mathrm{d} \Omega^{2} .
$$

The metric (187) recovers the one in the FLRW space-time by the choice $R=a(t) r$ and $\beta=-K r^{2}$, where $K$ is a cosmic curvature. In other cases the metric (187) describes a spherical inhomogeneity centered on the origin. We define the transverse Hubble function $H_{\perp}$ and the radial Hubble function $H_{\|}$, as $H_{\perp} \equiv \dot{R}^{\prime} / R^{\prime}$ and $H_{\perp}=\dot{R} / R$. The Einstein equations in the presence of non-relativistic matter (energy density $\rho_{m}$ ) give [63]

$$
\begin{aligned}
H_{\perp}^{2}+2 H_{\|} H_{\perp}-\frac{\beta}{R^{2}}-\frac{\beta^{\prime}}{R R^{\prime}} & =8 \pi G \rho_{m}, \\
6 \frac{\ddot{R}}{R}+2 H_{\perp}^{2}-2 \frac{\beta}{R^{2}}-2 H_{\|} H_{\perp}+\frac{\beta^{\prime}}{R R^{\prime}} & =-8 \pi G \rho_{m} .
\end{aligned}
$$

Eliminating the term $\rho_{m}$ from Eqs. (188) and (189), we obtain the relation $2 R \ddot{R}+\dot{R}^{2}=\beta(r)$. Integrating this equation, it follows that

$$
H_{\perp}^{2}=\frac{\alpha(r)}{R^{3}}+\frac{\beta(r)}{R^{2}},
$$


where $\alpha(r)$ is an arbitrary function of $r$. From this we can introduce the today's effective density parameters of matter and the spatial curvature, respectively, as

$$
\Omega_{m}^{(0)}(r) \equiv \frac{\alpha(r)}{R_{0}^{3} H_{\perp 0}^{2}}, \quad \Omega_{K}^{(0)}(r)=1-\Omega_{m}^{(0)}(r)=\frac{\beta(r)}{R_{0}^{2} H_{\perp 0}^{2}} .
$$

We define the time $t=0$ at the decoupling epoch (the redshift $z \simeq 1090)$ with $R(r, t=0)=0$. Introducing the conformal time $\eta$ as $\mathrm{d} \eta=(\sqrt{\beta} / R) \mathrm{d} t$, we obtain the following parametric solutions of Eqs. (188) and (189) for $\beta>0$ [63]:

$$
\begin{aligned}
& R=\frac{\alpha(r)}{2 \beta(r)}(\cosh \eta-1)=\frac{R_{0} \Omega_{m}^{(0)}(r)}{2\left[1-\Omega_{m}^{(0)}(r)\right]}(\cosh \eta-1), \\
& t=\frac{\alpha(r)}{2 \beta^{3 / 2}(r)}(\sinh \eta-\eta)=\frac{\Omega_{m}^{(0)}(r)}{2\left[1-\Omega_{m}^{(0)}(r)\right]^{3 / 2} H_{\perp 0}}(\sinh \eta-\eta) .
\end{aligned}
$$

The structure of the void with an under density can be accommodated by choosing $\Omega_{m}^{(0)}(r)$ and $h \equiv$ $H_{\perp 0} /\left(100 \mathrm{~km} \mathrm{sec}^{-1} \mathrm{Mpc}^{-1}\right)$ in the following form [598] (see also Ref. 63] for another choice)

$$
\begin{aligned}
\Omega_{m}^{(0)}(r) & =\Omega_{\text {out }}+\left(\Omega_{\text {in }}-\Omega_{\text {out }}\right) f\left(r, r_{0}, \Delta\right), \\
h(r) & =h_{\text {out }}+\left(h_{\text {in }}-h_{\text {out }}\right) f\left(r, r_{0}, \Delta\right),
\end{aligned}
$$

where the function $f\left(r, r_{0}, \Delta\right)=\left[1-\tanh \left(\left(r-r_{0}\right) / 2 \Delta\right)\right] /\left[1+\tanh \left(r_{0} / 2 \Delta\right)\right]$ describes a transition of a shell of radius $r_{0}$ and thickness $\Delta$ ("in" and "out" represent quantities inside and outside the void, respectively).

The trajectory of photons arriving at $r=0$ today is characterized by a path $t=\hat{t}(r)$ satisfying [590]

$$
\frac{\mathrm{d} \hat{t}}{\mathrm{~d} r}=-\frac{R^{\prime}(r, \hat{t})}{\sqrt{1+\beta}}
$$

Then one can show that the redshift $z=z(r)$ of photons obeys the differential equation 62.

$$
\frac{\mathrm{d} z}{\mathrm{~d} r}=(z+1) \frac{\dot{R}^{\prime}(r, \hat{t})}{\sqrt{1+\beta}}
$$

with $z(r=0)=0$. The luminosity distance $d_{L}(z)$ is related to the diameter distance $d_{A}(z)=R(r, \hat{t})$ according to the usual duality relation

$$
d_{L}(z)=(1+z)^{2} R(r, \hat{t}) .
$$

Now we are ready to confront the inhomogeneous LTB model with the SN Ia observations. From the requirement that the CMB acoustic peak is not spoiled we require that local density parameter $\Omega_{\text {in }}$ is in the range $0.1-0.3$, whereas $\Omega_{\text {out }}=1$ as predicted by inflation. The observed local value of $H$ is around $h_{\text {in }} \approx 0.7$, whereas outside the void one requires $h \approx 0.5$ to be consistent with $\Omega_{\text {out }}=1$. Then the two parameters $r_{0}$ and $\Delta$ are constrained by the SN Ia data. In Ref. [598] it was shown that the inhomogeneous LTB model can be consistent with the SN Ia data for $r_{0}=2.3 \pm 0.9 \mathrm{Gpc}$ and $\Delta / r_{0}>0.2$.

We note, however, that one can place other constraints on the void model. If we do not live around the center of the void, the observed CMB dipole becomes much larger than that allowed by observations. The maximum distance $r_{c}$ to the center is constrained to be smaller than 10-20 Mpc [590, 605]. Even if we happen to live very close to the center of the void, we observe distant off-centered galaxy clusters. Such off-centered clusters should see a large CMB dipole in their reference frame. For us this manifests itself observationally as a kinematic Sunyaev-Zeldovich effect. Using the observational data of only 9 clusters, the inhomogeneous LTB model with void sizes greater than $1.5 \mathrm{Gpc}$ can be ruled out [599, 604]. This is already in mild conflict with the constraint derived by the SN Ia data. It remains to see whether the void model can be ruled out or not in future observations.

\section{B. Backreaction of cosmological perturbations}

Let us finally discuss the possibility of realizing a real cosmic acceleration by the backreaction of inhomogeneities to the FLRW space-time. In general averaging the inhomogeneities and then solving the Einstein equations (the standard 
approach) might not be the same as solving the full inhomogeneous Einstein equations first and then averaging them. In other words, the expected value of a nonlinear function of $x$ is not the same as the nonlinear function of the expected value of $x$. The argument is complicated and controversial, so we mention the basic ideas only briefly. The readers who are interested in the detail of this line of research may have a look at the original papers [64 66, 614 628].

Let us decompose the Einstein tensor $G_{\mu \nu}$ and the energy-momentum tensor $T_{\mu \nu}$ into the background (0-th order FLRW Universe) and the perturbed parts, as $G_{\mu \nu}=G_{\mu \nu}^{(0)}+G_{\mu \nu}^{(1)}$ and $T_{\mu \nu}=T_{\mu \nu}^{(0)}+T_{\mu \nu}^{(1)}$, respectively. Then the (00) component of the Einstein equation (4) gives

$$
G_{00}^{(0)}=8 \pi G\left(T_{00}^{(0)}+T_{00}^{(1)}\right)-G_{00}^{(1)} .
$$

Identifying the average matter density at this order as $\langle\rho\rangle=T_{00}^{(0)}+T_{00}^{(1)}$ and averaging over Eq. (199), we obtain

$$
\left\langle G_{00}^{(0)}\right\rangle=8 \pi G\langle\rho\rangle-\left\langle G_{00}^{(1)}\right\rangle
$$

where $G_{00}^{(0)}=3 H^{2}$ in the FLRW background. This shows that $3 H^{2} \neq 8 \pi G\langle\rho\rangle$ in general because of the presence of the term $\left\langle G_{00}^{(1)}\right\rangle$. If we first average the metric as $\left\langle g_{\mu \nu}^{(1)}\right\rangle=0$, it then follows that $G_{00}^{(1)}=G_{00}\left(\left\langle g_{\mu \nu}^{(1)}\right\rangle\right)=0$ and hence $3 H^{2}=8 \pi G\langle\rho\rangle$. The above argument can be extended to the second order, i.e.

$$
\left\langle G_{00}^{(0)}\right\rangle=8 \pi G\langle\rho\rangle-\left\langle G_{00}^{(1)}+G_{00}^{(2)}\right\rangle
$$

The cosmological evolution depends on the averaging procedure. For example, if we take the average of the function $f(t, x)$ as 65 ]

$$
\langle f\rangle(t)=\frac{\int \mathrm{d}^{3} x \sqrt{\gamma(t, x)} f(t, x)}{\int \mathrm{d}^{3} x \sqrt{\gamma(t, x)}},
$$

where $\gamma$ corresponds to the determinant of the perturbed metric of spatial constant-time hypersurfaces, then the second-order term $G_{00}^{(2)}$ contributes to the expansion rate of the Universe (which is typically of the order of $10^{-5}$ ). If we use other ways of averaging, the amplitude of such a term is subject to change [616]. Also the results are affected by adding the contributions higher than the second order. In fact Ref. [614] pointed out the danger of arbitrarily stopping at some order by showing several examples in which many contributions cancel each other.

The backreaction scenario is very attractive if it really works, because it is the most economical way of explaining the cosmic acceleration without using dark energy. We hope that further progress will be expected in this direction.

\section{CONCLUSIONS}

We summarize the results presented in this review.

- The cosmological constant $\left(w_{\mathrm{DE}}=-1\right)$ is favored by a number of observations, but theoretically it is still challenging to explain why its energy scale is very small.

- Quintessence leads to the variation of the field equation of state in the region $w_{\phi}>-1$, but the current observations are not sufficient to distinguish between quintessence potentials.

- In k-essence it is possible to realize the cosmic acceleration by a field kinetic energy, while avoiding the instability problem associated with a phantom field. The k-essence models that aim to solve the coincidence problem inevitably leads to the superluminal propagation of the sound speed.

- In coupled dark energy models there is an upper bound on the strength of the coupling from the observations of CMB, large-scale structure and SN Ia.

- The generalized Chaplygin gas model allows the unified description of dark energy and dark matter, but it needs to be very close to the $\Lambda$ CDM model to explain the observed matter power spectrum. There is a class of viable unified models of dark energy and dark matter using a purely k-essence field.

- In $f(R)$ gravity and scalar-tensor theories it is possible to construct viable models that satisfy both cosmological and local gravity constraints. These models leave several interesting observational signatures such as the modifications to the matter power spectrum and to the weak lensing spectrum. 
- The dark energy models based on the Gauss-Bonnet term are in conflict with a number of observations and experiments in general and hence they are excluded as an alternative to the $\Lambda$ CDM model.

- The DGP model allows the self-acceleration of the Universe, but it is effectively ruled out from observational constraints and the ghost problem. However, some of the extension of works such as Galileon gravity allow the possibility for avoiding the ghost problem, while satisfying cosmological and local gravity constraints.

- The models based on the inhomogeneities in the distribution of matter allow the possibility for explaining the apparent accelerated expansion of the Universe. The void model can be consistent with the SN Ia data, but it is still challenging to satisfy all other constraints coming from the CMB and the kinematic Sunyaev-Zeldovich effect.

When the author submitted a review article [9] on dark energy to International Journal of Modern Physics D in March 2006, we wrote in concluding section that "over 900 papers with the words 'dark energy' in the title have appeared on the archives since 1998, and nearly 800 with the words 'cosmological constant' have appeared". Now in April 2010, I need to change the sentence to "over 2250 papers with the words 'dark energy' in the title have appeared on the archives since 1998, and nearly 1750 with the words 'cosmological constant' have appeared". This means that over 4000 papers about dark energy and cosmological constant have been already written, with more than 2300 papers over the past 4 years. Many cosmologists, astrophysicists, and particle physicists have extensively worked on this new field of research after the first discovery of the cosmic acceleration in 1998. We hope that the future progress of both theory and observations will provide some exciting clue to reveal the origin of dark energy.

\section{Acknowledgments}

I thank Sabino Matarrese to invite me to write this article in a chapter "dark energy: investigation and modeling" of a book published in Springer. I am also grateful to my all collaborators and colleagues with whom I discussed a lot about dark energy. I also thank Eiichiro Komatsu, Marek Kowalski, Elic Linder, Jun-Qing Xia, and Jun'ichi Yokoyama for permission to include figures from their papers. This work was supported by Grant-in-Aid for Scientific Research Fund of the JSPS (No. 30318802) and Grant-in-Aid for Scientific Research on Innovative Areas (No. 21111006).

[1] A. G. Riess et al. [Supernova Search Team Collaboration], Astron. J. 116, 1009 (1998).

[2] S. Perlmutter et al. [Supernova Cosmology Project Collaboration], Astrophys. J. 517, 565 (1999).

[3] D. Huterer and M. S. Turner, Phys. Rev. D 60, 081301 (1999).

[4] V. Sahni and A. A. Starobinsky, Int. J. Mod. Phys. D 9, 373 (2000).

[5] S. M. Carroll, Living Rev. Rel. 4, 1 (2001).

[6] P. J. E. Peebles and B. Ratra, Rev. Mod. Phys. 75, 559 (2003).

[7] T. Padmanabhan, Phys. Rept. 380, 235 (2003).

[8] V. Sahni, Lect. Notes Phys. 653, 141 (2004)

[9] E. J. Copeland, M. Sami and S. Tsujikawa, Int. J. Mod. Phys. D 15, 1753 (2006).

[10] R. Durrer and R. Maartens, Gen. Rel. Grav. 40, 301 (2008).

[11] R. R. Caldwell and M. Kamionkowski, Ann. Rev. Nucl. Part. Sci. 59, 397 (2009).

[12] L. Amendola and S. Tsujikawa, Dark energy-Theory and observations, Cambridge University Press (2010).

[13] P. Astier et al. [The SNLS Collaboration], Astron. Astrophys. 447, 31 (2006).

[14] A. G. Riess et al. [Supernova Search Team Collaboration], Astrophys. J. 607, 665 (2004).

[15] A. G. Riess et al., Astrophys. J. 659, 98 (2007).

[16] W. M. Wood-Vasey et al. [ESSENCE Collaboration], Astrophys. J. 666, 694 (2007).

[17] T. M. Davis et al., Astrophys. J. 666, 716 (2007).

[18] M. Kowalski et al. [Supernova Cosmology Project Collaboration], Astrophys. J. 686, 749 (2008).

[19] D. N. Spergel et al. [WMAP Collaboration], Astrophys. J. Suppl. 148, 175 (2003).

[20] D. N. Spergel et al. [WMAP Collaboration], Astrophys. J. Suppl. 170, 377 (2007).

[21] E. Komatsu et al. [WMAP Collaboration], Astrophys. J. Suppl. 180, 330 (2009).

[22] E. Komatsu et al., arXiv:1001.4538 [astro-ph.CO].

[23] D. J. Eisenstein et al. [SDSS Collaboration], Astrophys. J. 633, 560 (2005).

[24] W. J. Percival, S. Cole, D. J. Eisenstein, R. C. Nichol, J. A. Peacock, A. C. Pope and A. S. Szalay, Mon. Not. Roy. Astron. Soc. 381, 1053 (2007).

[25] W. J. Percival et al., Mon. Not. Roy. Astron. Soc. 401, 2148 (2010).

[26] S. Weinberg, Rev. Mod. Phys. 61, 1 (1989).

[27] S. Kachru, R. Kallosh, A. D. Linde and S. P. Trivedi, Phys. Rev. D 68, 046005 (2003). 
[28] Y. Fujii, Phys. Rev. D 26, 2580 (1982).

[29] L. H. Ford, Phys. Rev. D 35, 2339 (1987).

[30] C. Wetterich, Nucl. Phys B. 302, 668 (1988).

[31] B. Ratra and J. Peebles, Phys. Rev D 37, 321 (1988).

[32] T. Chiba, N. Sugiyama and T. Nakamura, Mon. Not. Roy. Astron. Soc. 289, L5 (1997).

[33] P. G. Ferreira and M. Joyce, Phys. Rev. Lett. 79, 4740 (1997).

[34] P. G. Ferreira and M. Joyce, Phys. Rev. D 58, 023503 (1998).

[35] E. J. Copeland, A. R. Liddle and D. Wands, Phys. Rev. D 57, 4686 (1998).

[36] R. R. Caldwell, R. Dave and P. J. Steinhardt, Phys. Rev. Lett. 80, 1582 (1998).

[37] I. Zlatev, L. M. Wang and P. J. Steinhardt, Phys. Rev. Lett. 82, 896 (1999).

[38] P. J. Steinhardt, L. M. Wang and I. Zlatev, Phys. Rev. D 59, 123504 (1999).

[39] T. Chiba, T. Okabe and M. Yamaguchi, Phys. Rev. D 62, 023511 (2000).

[40] C. Armendariz-Picon, V. F. Mukhanov and P. J. Steinhardt, Phys. Rev. Lett. 85, 4438 (2000).

[41] C. Armendariz-Picon, V. F. Mukhanov and P. J. Steinhardt, Phys. Rev. D 63, 103510 (2001).

[42] S. M. Carroll, Phys. Rev. Lett. 81, 3067 (1998).

[43] C. F. Kolda and D. H. Lyth, Phys. Lett. B 458, 197 (1999).

[44] A. Y. Kamenshchik, U. Moschella and V. Pasquier, Phys. Lett. B 511, 265 (2001).

[45] M. C. Bento, O. Bertolami and A. A. Sen, Phys. Rev. D 66, 043507 (2002).

[46] H. Sandvik, M. Tegmark, M. Zaldarriaga and I. Waga, Phys. Rev. D 69, 123524 (2004).

[47] S. Capozziello, Int. J. Mod. Phys. D 11, 483 (2002).

[48] S. Capozziello, S. Carloni and A. Troisi, Recent Res. Dev. Astron. Astrophys. 1, 625 (2003).

[49] S. Capozziello, S. Carloni and A. Troisi, Recent Res. Dev. Astron. Astrophys. 1, 625 (2003).

[50] S. Capozziello, V. F. Cardone, S. Carloni and A. Troisi, Int. J. Mod. Phys. D 12, 1969 (2003).

[51] S. M. Carroll, V. Duvvuri, M. Trodden and M. S. Turner, Phys. Rev. D 70, 043528 (2004).

[52] S. Nojiri and S. D. Odintsov, Phys. Rev. D 68, 123512 (2003).

[53] L. Amendola, Phys. Rev. D 60, 043501 (1999).

[54] J. P. Uzan, Phys. Rev. D 59, 123510 (1999).

[55] T. Chiba, Phys. Rev. D 60, 083508 (1999).

[56] N. Bartolo and M. Pietroni, Phys. Rev. D 61023518 (2000).

[57] F. Perrotta, C. Baccigalupi and S. Matarrese, Phys. Rev. D 61, 023507 (2000).

[58] G. R. Dvali, G. Gabadadze and M. Porrati, Phys. Lett. B 485, 208 (2000).

[59] K. Tomita, Astrophys. J. 529, 38 (2000).

[60] K. Tomita, Mon. Not. Roy. Astron. Soc. 326, 287 (2001).

[61] M. N. Celerier, Astron. Astrophys. 353, 63 (2000).

[62] H. Iguchi, T. Nakamura and K. i. Nakao, Prog. Theor. Phys. 108, 809 (2002).

[63] H. Alnes, M. Amarzguioui and O. Gron, Phys. Rev. D 73, 083519 (2006).

[64] S. Rasanen, JCAP 0402, 003 (2004).

[65] E. W. Kolb, S. Matarrese, A. Notari and A. Riotto, Phys. Rev. D 71, 023524 (2005).

[66] E. W. Kolb, S. Matarrese and A. Riotto, New J. Phys. 8, 322 (2006).

[67] S. Weinberg, Gravitation and Cosmology, Wiley and Sons, New York (1972).

[68] R. R. Caldwell, Phys. Lett. B 545, 23 (2002).

[69] A. A. Starobinsky, Grav. Cosmol. 6, 157 (2000).

[70] R. R. Caldwell, M. Kamionkowski and N. N. Weinberg, Phys. Rev. Lett. 91, 071301 (2003).

[71] J. D. Barrow, G. Galloway and F. Tipler, Mon. Not. Roy. astr. Soc. 223, 835 (1986).

[72] J. D. Barrow, Class. Quant. Grav. 21, L79 (2004).

[73] J. D. Barrow, Class. Quant. Grav. 21, 5619 (2004).

[74] H. Stefancic, Phys. Rev. D 71, 084024 (2005).

[75] S. Nojiri, S. D. Odintsov and S. Tsujikawa, Phys. Rev. D 71, 063004 (2005).

[76] I. H. Brevik and O. Gorbunova, Gen. Rel. Grav. 37, 2039 (2005).

[77] M. P. Dabrowski, Phys. Lett. B 625, 184 (2005).

[78] M. Sami, A. Toporensky, P. V. Tretjakov and S. Tsujikawa, Phys. Lett. B 619, 193 (2005).

[79] M. Bouhmadi-Lopez and J. A. Jimenez Madrid, JCAP 0505, 005 (2005).

[80] S. Nojiri and S. D. Odintsov, Phys. Lett. B 595, 1 (2004).

[81] M. Sami, P. Singh and S. Tsujikawa, Phys. Rev. D 74, 043514 (2006).

[82] D. Samart and B. Gumjudpai, Phys. Rev. D 76, 043514 (2007).

[83] L. Amendola and S. Tsujikawa, Phys. Lett. B 660, 125 (2008).

[84] J. Weller and A. J. Albrecht, Phys. Rev. D 65, 103512 (2002).

[85] M. Chevallier and D. Polarski, Int. J. Mod. Phys. D 10, 213 (2001).

[86] E. V. Linder, Phys. Rev. Lett. 90, 091301 (2003).

[87] H. K. Jassal, J. S. Bagla and T. Padmanabhan, Mon. Not. Roy. Astron. Soc. 356, L11 (2005).

[88] G. Efstathiou, Mon. Not. R. Astron. Soc. 342, 810 (2000).

[89] B. A. Bassett, M. Kunz, J. Silk and C. Ungarelli, Mon. Not. Roy. Astron. Soc. 336, 1217 (2002).

[90] B. A. Bassett, P. S. Corasaniti and M. Kunz, Astrophys. J. 617, L1 (2004).

[91] A. A. Starobinsky, JETP Lett. 68, 757 (1998). 
[92] T. D. Saini, S. Raychaudhury, V. Sahni and A. A. Starobinsky, Phys. Rev. Lett. 85, 1162 (2000).

[93] U. Alam, V. Sahni, T. D. Saini and A. A. Starobinsky, Mon. Not. Roy. Astron. Soc. 344, 1057 (2003).

[94] U. Alam, V. Sahni, T. D. Saini and A. A. Starobinsky, Mon. Not. Roy. Astron. Soc. 354, 275 (2004).

[95] I. Maor, R. Brustein, J. McMahon and P. J. Steinhardt, Phys. Rev. D 65, 123003 (2002).

[96] P. S. Corasaniti and E. J. Copeland, Phys. Rev. D 67, 063521 (2003).

[97] Y. Wang and P. Mukherjee, Astrophys. J. 606, 654 (2004).

[98] S. Nesseris and L. Perivolaropoulos, Phys. Rev. D 70, 043531 (2004).

[99] R. Lazkoz, S. Nesseris and L. Perivolaropoulos, JCAP 0511, 010 (2005).

[100] A. Upadhye, M. Ishak and P. J. Steinhardt, Phys. Rev. D 72, 063501 (2005).

[101] Z. K. Guo, N. Ohta and Y. Z. Zhang, Phys. Rev. D 72, 023504 (2005).

[102] Y. Wang and P. Mukherjee, Astrophys. J. 650, 1 (2006).

[103] S. Nesseris and L. Perivolaropoulos, JCAP 0702, 025 (2007).

[104] R. Crittenden, E. Majerotto and F. Piazza, Phys. Rev. Lett. 98, 251301 (2007).

[105] K. Ichikawa and T. Takahashi, JCAP 0702, 001 (2007).

[106] G. B. Zhao, J. Q. Xia, B. Feng and X. Zhang, Int. J. Mod. Phys. D 16, 1229 (2007).

[107] G. B. Zhao, J. Q. Xia, H. Li, C. Tao, J. M. Virey, Z. H. Zhu and X. Zhang, Phys. Lett. B 648, 8 (2007).

[108] B. A. Bassett et al., JCAP 0807, 007 (2008).

[109] K. Ichikawa and T. Takahashi, JCAP 0804, 027 (2008).

[110] A. G. Riess et al., Astrophys. J. 699, 539 (2009).

[111] S. H. Suyu et al., Astrophys. J. 711, 201 (2010).

[112] R. K. Sachs and A. M. Wolfe, Astrophys. J. 147, 73 (1967).

[113] A. A. Starobinsky, Phys. Lett. B 91 (1980) 99.

[114] D. Kazanas, Astrophys. J. 241 L59 (1980).

[115] K. Sato, Mon. Not. R. Astron. Soc. 195, 467 (1981).

[116] A. H. Guth, Phys. Rev. D 23, 347 (1981).

[117] G. F. Smoot et al., Astrophys. J. 396, L1 (1992).

[118] A. R. Liddle and D. H. Lyth, Cosmological inflation and large-scale structure, Cambridge University Press (2000).

[119] B. A. Bassett, S. Tsujikawa and D. Wands, Rev. Mod. Phys. 78, 537 (2006).

[120] L. Page et al. [WMAP Collaboration], Astrophys. J. Suppl. 148, 233 (2003).

[121] W. Hu and N. Sugiyama, Astrophys. J. 444, 489 (1995).

[122] W. Hu and N. Sugiyama, Astrophys. J. 471, 542 (1996).

[123] G. Efstathiou and J. R. Bond, Mon. Not. Roy. Astron. Soc. 304, 75 (1999).

[124] M. Doran and M. Lilley, Mon. Not. Roy. Astron. Soc. 330, 965 (2002).

[125] A. Melchiorri, L. Mersini, C. J. Odman and M. Trodden, Phys. Rev. D 68, 043509 (2003).

[126] S. Hannestad and E. Mortsell, Phys. Rev. D 66, 063508 (2002).

[127] J. Weller and A. M. Lewis, Mon. Not. Roy. Astron. Soc. 346, 987 (2003).

[128] P. S. Corasaniti, T. Giannantonio and A. Melchiorri, Phys. Rev. D 71, 123521 (2005).

[129] W. Lee and K. W. Ng, Phys. Rev. D 67, 107302 (2003).

[130] Y. Wang and M. Tegmark, Phys. Rev. Lett. 92, 241302 (2004).

[131] D. Rapetti, S. W. Allen and J. Weller, Mon. Not. Roy. Astron. Soc. 360, 555 (2005).

[132] L. Pogosian, P. S. Corasaniti, C. Stephan-Otto, R. Crittenden and R. Nichol, Phys. Rev. D 72, 103519 (2005).

[133] J. Q. Xia, G. B. Zhao, B. Feng, H. Li and X. Zhang, Phys. Rev. D 73, 063521 (2006).

[134] D. J. Eisenstein and W. Hu, Astrophys. J. 496, 605 (1998).

[135] M. Shoji, D. Jeong and E. Komatsu, Astrophys. J. 693, 1404 (2009).

[136] B. Feng, X. L. Wang and X. M. Zhang, Phys. Lett. B 607, 35 (2005).

[137] M. Tegmark et al. [SDSS Collaboration], Phys. Rev. D 69, 103501 (2004).

[138] U. Seljak et al., Phys. Rev. D 71, 103515 (2005).

[139] M. Tegmark et al. [SDSS Collaboration], Phys. Rev. D 74, 123507 (2006).

[140] D. Hooper and S. Dodelson, Astropart. Phys. 27, 113 (2007).

[141] M. Oguri and K. Takahashi, Phys. Rev. D 73, 123002 (2006).

[142] S. Basilakos and L. Perivolaropoulos, Mon. Not. Roy. Astron. Soc. 391, 411 (2008).

[143] Y. Wang, Phys. Rev. D 78, 123532 (2008).

[144] R. Tsutsui, T. Nakamura, D. Yonetoku, T. Murakami, S. Tanabe, Y. Kodama and K. Takahashi, Mon. Not. Roy. Astron. Soc. 394, L31 (2009).

[145] B. Jain and A. Taylor, Phys. Rev. Lett. 91, 141302 (2003).

[146] M. Takada and B. Jain, Mon. Not. Roy. Astron. Soc. 348, 897 (2004).

[147] M. Ishak, Mon. Not. Roy. Astron. Soc. 363, 469 (2005).

[148] C. Schimd et al., Astron. Astrophys. 463, 405 (2007).

[149] M. Takada and S. Bridle, New J. Phys. 9, 446 (2007).

[150] L. Hollenstein, D. Sapone, R. Crittenden and B. M. Schaefer, JCAP 0904, 012 (2009).

[151] D. Bailin and A. Love, Supersymmetric gauge field theory and string theory, Institute of Physics Publishing (1994).

[152] M. Green, J. H. Schwarz and E. Witten, Superstring theory, Cambridge University Press, Cambridge (1987).

[153] M. Dine, R. Rohm, N. Seiberg and E. Witten, Phys. Lett. B 156, 55 (1985).

[154] I. Affleck, M. Dine and N. Seiberg, Nucl. Phys. B 256, 557 (1985). 
[155] L. Susskind, arXiv:hep-th/0302219.

[156] B. Carter, Large number coincidences and the anthropic principle in cosmology, IAU Symposium 63: Con- frontation of cosmological theories with observational data, 291 (1974).

[157] J. Barrow and F. Tipler, The cosmological anthropic principle, Oxford University Press, Oxford (1988).

[158] S. Weinberg, Phys. Rev. Lett. 59, 2607 (1987).

[159] J. Garriga and A. Vilenkin, Phys. Rev. D 61, 083502 (2000).

[160] F. Denef and M. R. Douglas, JHEP 0405, 072 (2004).

[161] J. Garriga, A. D. Linde and A. Vilenkin, Phys. Rev. D 69, 063521 (2004).

[162] R. Blumenhagen et al., Nucl. Phys. B 713, 83 (2005).

[163] A. D. Linde, Rept. Prog. Phys. 47, 925 (1984).

[164] J. D. Brown and C. Teitelboim, Phys. Lett. B 195, 177 (1987).

[165] J. D. Brown and C. Teitelboim, Nucl. Phys. B 297, 787 (1988).

[166] R. Bousso, Gen. Rel. Grav. 40, 607 (2008).

[167] R. Bousso and J. Polchinski, JHEP 0006, 006 (2000).

[168] C. de Rham, G. Dvali, S. Hofmann, J. Khoury, O. Pujolas, M. Redi and A. J. Tolley, Phys. Rev. Lett. 100, 251603 (2008).

[169] N. Afshordi, arXiv:0807.2639 [astro-ph].

[170] T. Padmanabhan, Gen. Rel. Grav. 40, 529 (2008).

[171] T. Padmanabhan, arXiv:0807.2356 [gr-qc].

[172] S. W. Hawking, Phys. Lett. B 134, 403 (1984).

[173] S. Kachru, M. B. Schulz and E. Silverstein, Phys. Rev. D 62, 045021 (2000).

[174] N. Arkani-Hamed, S. Dimopoulos, N. Kaloper and R. Sundrum, Phys. Lett. B 480, 193 (2000).

[175] J. L. Feng, J. March-Russell, S. Sethi and F. Wilczek, Nucl. Phys. B 602, 307 (2001).

[176] S. H. H. Tye and I. Wasserman, Phys. Rev. Lett. 86, 1682 (2001).

[177] J. Garriga and A. Vilenkin, Phys. Rev. D 64, 023517 (2001).

[178] J. Yokoyama, Phys. Rev. Lett. 88, 151302 (2002).

[179] C. P. Burgess, R. Kallosh and F. Quevedo, JHEP 0310, 056 (2003).

[180] Y. Aghababaie, C. P. Burgess, S. L. Parameswaran and F. Quevedo, Nucl. Phys. B 680, 389 (2004).

[181] S. Mukohyama and L. Randall, Phys. Rev. Lett. 92, 211302 (2004).

[182] M. Ahmed, S. Dodelson, P. B. Greene and R. Sorkin, Phys. Rev. D 69, 103523 (2004).

[183] G. L. Kane, M. J. Perry and A. N. Zytkow, Phys. Lett. B 609, 7 (2005).

[184] A. D. Dolgov and F. R. Urban, Phys. Rev. D 77, 083503 (2008).

[185] A. de la Macorra and G. Piccinelli, Phys. Rev. D 61, 123503 (2000).

[186] S. C. C. Ng, N. J. Nunes and F. Rosati, Phys. Rev. D 64, 083510 (2001).

[187] R. R. Caldwell and E. V. Linder, Phys. Rev. Lett. 95, 141301 (2005).

[188] P. Binetruy, Phys. Rev. D 60, 063502 (1999).

[189] P. Brax and J. Martin, Phys. Lett. B 468, 40 (1999).

[190] A. D. Linde, "Inflation and quantum cosmology", in: Three hundred years of gravitation, Eds.: S. W. Hawking and W. Israel, Cambridge Univeristy Press, 604 (1987).

[191] R. Kallosh et al., JCAP 0310, 015 (2003).

[192] J. A. Frieman, C. T. Hill, A. Stebbins and I. Waga, Phys. Rev. Lett. 75, 2077 (1995).

[193] Y. Nomura, T. Watari and T. Yanagida, Phys. Lett. B 484, 103 (2000).

[194] K. Choi, Phys. Rev. D 62, 043509 (2000).

[195] J. E. Kim and H. P. Nilles, Phys. Lett. B 553, 1 (2003).

[196] L. J. Hall, Y. Nomura and S. J. Oliver, Phys. Rev. Lett. 95, 141302 (2005).

[197] E. J. Copeland, N. J. Nunes and F. Rosati, Phys. Rev. D 62, 123503 (2000).

[198] P. K. Townsend, JHEP 0111, 042 (2001).

[199] S. Hellerman, N. Kaloper and L. Susskind, JHEP 0106, 003 (2001).

[200] R. Kallosh, A. D. Linde, S. Prokushkin and M. Shmakova, Phys. Rev. D 65, 105016 (2002).

[201] R. Kallosh, A. D. Linde, S. Prokushkin and M. Shmakova, Phys. Rev. D 66, 123503 (2002).

[202] P. Fre, M. Trigiante and A. Van Proeyen, Class. Quant. Grav. 19, 4167 (2002).

[203] L. A. Boyle, R. R. Caldwell and M. Kamionkowski, Phys. Lett. B 545, 17 (2002).

[204] M. Gasperini, F. Piazza and G. Veneziano, Phys. Rev. D 65, 023508 (2002).

[205] T. Damour, F. Piazza and G. Veneziano, Phys. Rev. Lett. 89, 081601 (2002).

[206] A. J. Albrecht, C. P. Burgess, F. Ravndal and C. Skordis, Phys. Rev. D 65, 123507 (2002).

[207] C. P. Burgess, C. Nunez, F. Quevedo, G. Tasinato and I. Zavala, JHEP 0308, 056 (2003).

[208] C. P. Burgess, Annals Phys. 313, 283 (2004).

[209] E. V. Linder, Phys. Rev. D 73, 063010 (2006).

[210] V. Sahni, T. D. Saini, A. A. Starobinsky and U. Alam, JETP Lett. 77, 201 (2003).

[211] V. Sahni, A. Shafieloo and A. A. Starobinsky, Phys. Rev. D 78, 103502 (2008).

[212] T. Nakamura and T. Chiba, Mon. Not. Roy. Astron. Soc. 306, 696 (1999).

[213] T. Chiba and T. Nakamura, Phys. Rev. D 62, 121301 (2000).

[214] R. Bean, S. H. Hansen and A. Melchiorri, Phys. Rev. D 64, 103508 (2001).

[215] T. Barreiro, E. J. Copeland and N. J. Nunes, Phys. Rev. D 61, 127301 (2000).

[216] V. Sahni and L. M. Wang, Phys. Rev. D 62, 103517 (2000). 
[217] A. J. Albrecht and C. Skordis, Phys. Rev. Lett. 84, 2076 (2000).

[218] S. Dodelson, M. Kaplinghat and E. Stewart, Phys. Rev. Lett. 85, 5276 (2000).

[219] L. A. Urena-Lopez and T. Matos, Phys. Rev. D 62, 081302 (2000).

[220] A. A. Sen and S. Sethi, Phys. Lett. B 532, 159 (2002).

[221] D. Blais and D. Polarski, Phys. Rev. D 70, 084008 (2004).

[222] A. A. Coley and R. J. van den Hoogen, Phys. Rev. D 62, 023517 (2000).

[223] S. A. Kim, A. R. Liddle and S. Tsujikawa, Phys. Rev. D 72, 043506 (2005).

[224] A. R. Liddle, A. Mazumdar and F. E. Schunck, Phys. Rev. D 58, 061301 (1998).

[225] J. Ohashi and S. Tsujikawa, Phys. Rev. D 80, 103513 (2009).

[226] P. J. E. Peebles and A. Vilenkin, Phys. Rev. D 59, 063505 (1999).

[227] G. N. Felder, L. Kofman and A. D. Linde, Phys. Rev. D 59, 123523 (1999).

[228] M. Giovannini, Class. Quant. Grav. 16, 2905 (1999).

[229] M. Peloso and F. Rosati, JHEP 9912, 026 (1999).

[230] A. B. Kaganovich, Phys. Rev. D 63, 025022 (2001).

[231] M. Yahiro, G. J. Mathews, K. Ichiki, T. Kajino and M. Orito, Phys. Rev. D 65, 063502 (2002).

[232] K. Dimopoulos and J. W. F. Valle, Astropart. Phys. 18, 287 (2002).

[233] N. J. Nunes and E. J. Copeland, Phys. Rev. D 66, 043524 (2002).

[234] K. Dimopoulos, Phys. Rev. D 68, 123506 (2003).

[235] H. Tashiro, T. Chiba and M. Sasaki, Class. Quant. Grav. 21, 1761 (2004).

[236] M. Sami and V. Sahni, Phys. Rev. D 70, 083513 (2004).

[237] R. Rosenfeld and J. A. Frieman, JCAP 0509, 003 (2005).

[238] A. R. Liddle and L. A. Urena-Lopez, Phys. Rev. Lett. 97, 161301 (2006).

[239] I. P. Neupane, Class. Quant. Grav. 25, 125013 (2008).

[240] C. Armendariz-Picon, T. Damour and V. F. Mukhanov, Phys. Lett. B 458, 209 (1999).

[241] M. Gasperini and G. Veneziano, Astropart. Phys. 1, 317 (1993).

[242] M. Gasperini and G. Veneziano, Phys. Rept. 373, 1 (2003).

[243] S. M. Carroll, M. Hoffman and M. Trodden, Phys. Rev. D 68, 023509 (2003).

[244] J. M. Cline, S. Jeon and G. D. Moore, Phys. Rev. D 70, 043543 (2004).

[245] N. Arkani-Hamed, H. C. Cheng, M. A. Luty and S. Mukohyama, JHEP 0405, 074 (2004).

[246] F. Piazza and S. Tsujikawa, JCAP 0407, 004 (2004).

[247] M. R. Garousi, Nucl. Phys. B 584, 284 (2000).

[248] A. Sen, JHEP 0204, 048 (2002).

[249] G. W. Gibbons, Phys. Lett. B 537, 1 (2002).

[250] M. Fairbairn and M. H. G. Tytgat, Phys. Lett. B 546, 1 (2002).

[251] A. Feinstein, Phys. Rev. D 66, 063511 (2002).

[252] L. Kofman and A. D. Linde, JHEP 0207, 004 (2002).

[253] T. Padmanabhan, Phys. Rev. D 66, 021301 (2002).

[254] L. R. W. Abramo and F. Finelli, Phys. Lett. B 575, 165 (2003).

[255] J. M. Aguirregabiria and R. Lazkoz, Phys. Rev. D 69, 123502 (2004).

[256] M. R. Garousi, M. Sami and S. Tsujikawa, Phys. Rev. D 70, 043536 (2004).

[257] E. J. Copeland, M. R. Garousi, M. Sami and S. Tsujikawa, Phys. Rev. D 71, 043003 (2005).

[258] G. Calcagni and A. R. Liddle, Phys. Rev. D 74, 043528 (2006).

[259] E. Silverstein and D. Tong, Phys. Rev. D 70, 103505 (2004).

[260] M. Alishahiha, E. Silverstein and D. Tong, Phys. Rev. D 70, 123505 (2004).

[261] J. Martin and M. Yamaguchi, Phys. Rev. D 77, 123508 (2008).

[262] Z. K. Guo and N. Ohta, JCAP 0804, 035 (2008).

[263] E. J. Copeland, S. Mizuno and M. Shaeri, arXiv:1003.2881 [hep-th].

[264] P. Singh, M. Sami and N. Dadhich, Phys. Rev. D 68, 023522 (2003).

[265] M. Sami and A. Toporensky, Mod. Phys. Lett. A 19, 1509 (2004).

[266] M. P. Dabrowski, T. Stachowiak and M. Szydlowski, Phys. Rev. D 68, 103519 (2003).

[267] E. Elizalde, S. Nojiri and S. D. Odintsov, Phys. Rev. D 70, 043539 (2004).

[268] Z. K. Guo, Y. S. Piao, X. M. Zhang and Y. Z. Zhang, Phys. Lett. B 608, 177 (2005).

[269] M. z. Li, B. Feng and X. m. Zhang, JCAP 0512, 002 (2005).

[270] H. Wei, R. G. Cai and D. F. Zeng, Class. Quant. Grav. 22, 3189 (2005).

[271] I. Y. Aref'eva, A. S. Koshelev and S. Y. Vernov, Phys. Rev. D 72, 064017 (2005).

[272] H. Wei and R. G. Cai, Phys. Rev. D 72, 123507 (2005).

[273] R. G. Cai and A. Wang, JCAP 0503, 002 (2005).

[274] G. B. Zhao, J. Q. Xia, M. Li, B. Feng and X. Zhang, Phys. Rev. D 72, 123515 (2005).

[275] X. F. Zhang, H. Li, Y. S. Piao and X. M. Zhang, Mod. Phys. Lett. A 21, 231 (2006).

[276] W. Zhao, Phys. Rev. D 73, 123509 (2006).

[277] Z. K. Guo, Y. S. Piao, X. Zhang and Y. Z. Zhang, Phys. Rev. D 74, 127304 (2006).

[278] S. Nojiri and S. D. Odintsov, Gen. Rel. Grav. 38, 1285 (2006).

[279] Y. f. Cai, M. z. Li, J. X. Lu, Y. S. Piao, T. t. Qiu and X. m. Zhang, Phys. Lett. B 651, 1 (2007).

[280] Y. F. Cai, E. N. Saridakis, M. R. Setare and J. Q. Xia, arXiv:0909.2776 [hep-th]. 
[281] A. Vikman, Phys. Rev. D 71, 023515 (2005).

[282] J. Garriga and V. F. Mukhanov, Phys. Lett. B 458, 219 (1999).

[283] C. Bonvin, C. Caprini and R. Durrer, Phys. Rev. Lett. 97, 081303 (2006).

[284] M. Malquarti, E. J. Copeland, A. R. Liddle and M. Trodden, Phys. Rev. D 67, 123503 (2003).

[285] C. Wetterich, Astron. Astrophys. 301, 321 (1995).

[286] L. Amendola, Phys. Rev. D 60, 043501 (1999).

[287] L. Amendola, Phys. Rev. D 62, 043511 (2000).

[288] D. J. Holden and D. Wands, Phys. Rev. D 61, 043506 (2000).

[289] G. Huey and B. D. Wandelt, Phys. Rev. D 74, 023519 (2006).

[290] S. Das, P. S. Corasaniti and J. Khoury, Phys. Rev. D 73, 083509 (2006).

[291] C. Brans and R. H. Dicke, Phys. Rev. 124, 925 (1961).

[292] S. Tsujikawa, K. Uddin, S. Mizuno, R. Tavakol and J. Yokoyama, Phys. Rev. D 77, 103009 (2008).

[293] W. Zimdahl, D. Pavon and L. P. Chimento, Phys. Lett. B521, 133 (2001).

[294] W. Zimdahl and D. Pavon, Gen. Rel. Grav. 35, 413 (2003).

[295] L. P. Chimento, A. S. Jakubi, D. Pavon and W. Zimdahl, Phys. Rev. D 67, 083513 (2003).

[296] N. Dalal, K. Abazajian, E. E. Jenkins and A. V. Manohar, Phys. Rev. Lett. 87, 141302 (2001).

[297] L. Amendola, G. Camargo Campos and R. Rosenfeld, Phys. Rev. D 75, 083506 (2007).

[298] Z. K. Guo, N. Ohta and S. Tsujikawa, Phys. Rev. D 76, 023508 (2007).

[299] C. G. Boehmer, G. Caldera-Cabral, R. Lazkoz and R. Maartens, Phys. Rev. D 78, 023505 (2008).

[300] J. Valiviita, E. Majerotto and R. Maartens, JCAP 0807, 020 (2008).

[301] C. G. Boehmer, G. Caldera-Cabral, N. Chan, R. Lazkoz and R. Maartens, arXiv:0911.3089 [gr-qc].

[302] D. Pavon, S. Sen and W. Zimdahl, JCAP 0405, 009 (2004).

[303] R. G. Cai and A. Wang, JCAP 0503, 002 (2005).

[304] Z. K. Guo, R. G. Cai and Y. Z. Zhang, JCAP 0505, 002 (2005).

[305] M. Nishiyama, M. a. Morita and M. Morikawa, arXiv:astro-ph/0403571.

[306] B. Gumjudpai, T. Naskar, M. Sami and S. Tsujikawa, JCAP 0506, 007 (2005).

[307] T. Koivisto, Phys. Rev. D 72, 043516 (2005).

[308] S. del Campo, R. Herrera, G. Olivares and D. Pavon, Phys. Rev. D 74, 023501 (2006).

[309] S. Lee, G. C. Liu and K. W. Ng, Phys. Rev. D 73, 083516 (2006).

[310] H. M. Sadjadi and M. Alimohammadi, Phys. Rev. D 74, 103007 (2006).

[311] O. Bertolami, F. Gil Pedro and M. Le Delliou, Phys. Lett. B 654, 165 (2007).

[312] R. Mainini and S. Bonometto, JCAP 0706, 020 (2007).

[313] H. Wei and S. N. Zhang, Phys. Lett. B 644, 7 (2007).

[314] T. Fukuyama, M. Morikawa and T. Tatekawa, JCAP 0806, 033 (2008).

[315] G. Caldera-Cabral, R. Maartens and L. A. Urena-Lopez, Phys. Rev. D 79, 063518 (2009).

[316] G. Caldera-Cabral, R. Maartens and B. M. Schaefer, JCAP 0907, 027 (2009).

[317] E. Majerotto, J. Valiviita and R. Maartens, Mon. Not. Roy. Astron. Soc. 402, 2344 (2010).

[318] H. Wei, arXiv:1004.0492 [gr-qc].

[319] L. Amendola and D. Tocchini-Valentini, Phys. Rev. D 66, 043528 (2002).

[320] L. Amendola, Phys. Rev. D 69, 103524 (2004).

[321] J. Peebles, The large-scale structure of the universe, Princeton University Press (1980).

[322] L. M. Wang and P. J. Steinhardt, Astrophys. J. 508, 483 (1998).

[323] E. V. Linder, Phys. Rev. D 72, 043529 (2005).

[324] C. Di Porto and L. Amendola, Phys. Rev. D 77, 083508 (2008).

[325] L. Amendola, Phys. Rev. Lett. 93, 181102 (2004).

[326] L. Amendola, S. Tsujikawa and M. Sami, Phys. Lett. B 632, 155 (2006).

[327] S. Tsujikawa, Phys. Rev. D 72, 083512 (2005).

[328] P. Q. Hung, arXiv:hep-ph/0010126.

[329] P. Gu, X. Wang and X. Zhang, Phys. Rev. D 68, 087301 (2003).

[330] R. Fardon, A. E. Nelson and N. Weiner, JCAP 0410, 005 (2004).

[331] R. D. Peccei, Phys. Rev. D 71, 023527 (2005).

[332] R. Takahashi and M. Tanimoto, Phys. Lett. B 633, 675 (2006).

[333] N. Afshordi, M. Zaldarriaga and K. Kohri, Phys. Rev. D 72, 065024 (2005).

[334] A. W. Brookfield, C. van de Bruck, D. F. Mota and D. Tocchini-Valentini, Phys. Rev. Lett. 96, 061301 (2006).

[335] A. W. Brookfield, C. van de Bruck, D. F. Mota and D. Tocchini-Valentini, Phys. Rev. D 73, 083515 (2006).

[336] L. Amendola, M. Baldi and C. Wetterich, Phys. Rev. D 78, 023015 (2008).

[337] K. Ichiki and Y. Y. Keum, JHEP 0806, 058 (2008).

[338] K. Bamba, C. Q. Geng and S. H. Ho, JCAP 0809, 001 (2008).

[339] S. Tsujikawa and M. Sami, Phys. Lett. B 603, 113 (2004).

[340] L. Amendola, M. Quartin, S. Tsujikawa and I. Waga, Phys. Rev. D 74, 023525 (2006).

[341] P. Binetruy, C. Deffayet and D. Langlois, Nucl. Phys. B 565, 269 (2000).

[342] T. Shiromizu, K. i. Maeda and M. Sasaki, Phys. Rev. D 62, 024012 (2000).

[343] L. Randall and R. Sundrum, Phys. Rev. Lett. 83, 3370 (1999).

[344] L. Randall and R. Sundrum, Phys. Rev. Lett. 83, 4690 (1999). 
[345] K. i. Maeda, Phys. Rev. D 64, 123525 (2001).

[346] S. Mizuno and K. i. Maeda, Phys. Rev. D 64, 123521 (2001).

[347] E. J. Copeland, S. J. Lee, J. E. Lidsey and S. Mizuno, Phys. Rev. D 71, 023526 (2005).

[348] S. Tsujikawa, Phys. Rev. D 73, 103504 (2006).

[349] L. Amendola and D. Tocchini-Valentini, Phys. Rev. D 64, 043509 (2001).

[350] J. Khoury and A. Weltman, Phys. Rev. Lett. 93, 171104 (2004).

[351] J. Khoury and A. Weltman, Phys. Rev. D 69, 044026 (2004).

[352] T. Tamaki and S. Tsujikawa, Phys. Rev. D 78, 084028 (2008).

[353] C. M. Will, Living Rev. Rel. 4, 4 (2001).

[354] C. M. Will, Living Rev. Rel. 9, 3 (2005).

[355] P. Brax, C. van de Bruck, A. C. Davis, J. Khoury and A. Weltman, Phys. Rev. D 70, 123518 (2004).

[356] S. S. Gubser and J. Khoury, Phys. Rev. D 70, 104001 (2004).

[357] H. Wei and R. G. Cai, Phys. Rev. D 71, 043504 (2005).

[358] D. F. Mota and D. J. Shaw, Phys. Rev. Lett. 97, 151102 (2006).

[359] P. Brax, C. van de Bruck, A. C. Davis, D. F. Mota and D. J. Shaw, Phys. Rev. D 76, 124034 (2007).

[360] P. Brax, C. van de Bruck and A. C. Davis, Phys. Rev. Lett. 99, 121103 (2007).

[361] D. F. Mota and D. J. Shaw, Phys. Rev. D 75, 063501 (2007).

[362] A. C. Davis, C. A. O. Schelpe and D. J. Shaw, Phys. Rev. D 80, 064016 (2009).

[363] L. Hui, A. Nicolis and C. Stubbs, Phys. Rev. D 80, 104002 (2009).

[364] Y. Fujii et al., Nucl. Phys. B 573, 377 (2000).

[365] M. T. Murphy et al., Mon. Not. Roy. Astron. Soc. 327, 1208 (2001).

[366] J. K. Webb et al., Phys. Rev. Lett. 87, 091301 (2001).

[367] H. Chand, R. Srianand, P. Petitjean and B. Aracil, Astron. Astrophys. 417, 853 (2004).

[368] M. T. Murphy, J. K. Webb, V. V. Flambaum and S. J. Curran, Astrophys. Space Sci. 283, 577 (2003).

[369] J. D. Bekenstein, Phys. Rev. D 25, 1527 (1982).

[370] H. B. Sandvik, J. D. Barrow and J. Magueijo, Phys. Rev. Lett. 88, 031302 (2002).

[371] G. R. Dvali and M. Zaldarriaga, Phys. Rev. Lett. 88, 091303 (2002).

[372] T. Chiba and K. Kohri, Prog. Theor. Phys. 107, 631 (2002).

[373] J. P. Uzan, Rev. Mod. Phys. 75, 403 (2003).

[374] D. Parkinson, B. A. Bassett and J. D. Barrow, Phys. Lett. B 578, 235 (2004).

[375] S. Lee, K. A. Olive and M. Pospelov, Phys. Rev. D 70, 083503 (2004).

[376] E. J. Copeland, N. J. Nunes and M. Pospelov, Phys. Rev. D 69, 023501 (2004).

[377] D. F. Mota and J. D. Barrow, Phys. Lett. B 581, 141 (2004).

[378] M. R. Garousi, M. Sami and S. Tsujikawa, Phys. Rev. D 71, 083005 (2005).

[379] P. P. Avelino, L. M. G. Beca, J. P. M. de Carvalho, C. J. A. Martins and E. J. Copeland, Phys. Rev. D 69, 041301 (2004).

[380] L. Amendola, F. Finelli, C. Burigana and D. Carturan, JCAP 0307, 005 (2003).

[381] L. Amendola, I. Waga and F. Finelli, JCAP 0511, 009 (2005).

[382] R. J. Scherrer, Phys. Rev. Lett. 93, 011301 (2004).

[383] D. Giannakis and W. Hu, Phys. Rev. D 72, 063502 (2005).

[384] C. Armendariz-Picon and E. A. Lim, JCAP 0508, 007 (2005).

[385] D. Bertacca, S. Matarrese and M. Pietroni, Mod. Phys. Lett. A 22, 2893 (2007).

[386] D. Bertacca, N. Bartolo, A. Diaferio and S. Matarrese, JCAP 0810, 023 (2008).

[387] O. F. Piattella, D. Bertacca, M. Bruni and D. Pietrobon, JCAP 1001, 014 (2010).

[388] Y. Urakawa and T. Kobayashi, arXiv:0907.1191 [astro-ph.CO].

[389] O. Bertolami, C. G. Boehmer, T. Harko and F. S. N. Lobo, Phys. Rev. D 75, 104016 (2007).

[390] O. Bertolami and J. Paramos, Phys. Rev. D 77, 084018 (2008).

[391] V. Faraoni, Phys. Rev. D 80, 124040 (2009).

[392] M. E. Soussa and R. P. Woodard, Gen. Rel. Grav. 36, 855 (2004).

[393] G. Allemandi, A. Borowiec and M. Francaviglia, Phys. Rev. D 70, 103503 (2004).

[394] D. A. Easson, Int. J. Mod. Phys. A 19, 5343 (2004).

[395] R. Dick, Gen. Rel. Grav. 36, 217 (2004).

[396] G. Allemandi, A. Borowiec and M. Francaviglia, Phys. Rev. D 70, 043524 (2004).

[397] S. Carloni, P. K. S. Dunsby, S. Capozziello and A. Troisi, Class. Quant. Grav. 22, 4839 (2005).

[398] A. D. Dolgov and M. Kawasaki, Phys. Lett. B 573, 1 (2003).

[399] L. Amendola, D. Polarski and S. Tsujikawa, Phys. Rev. Lett. 98, 131302 (2007).

[400] L. Amendola, D. Polarski and S. Tsujikawa, Int. J. Mod. Phys. D 16, 1555 (2007).

[401] T. Chiba, Phys. Lett. B 575, 1 (2003).

[402] G. J. Olmo, Phys. Rev. Lett. 95, 261102 (2005).

[403] G. J. Olmo, Phys. Rev. D 72, 083505 (2005).

[404] I. Navarro and K. Van Acoleyen, JCAP 0702, 022 (2007).

[405] A. L. Erickcek, T. L. Smith and M. Kamionkowski, Phys. Rev. D 74, 121501 (2006).

[406] T. Chiba, T. L. Smith and A. L. Erickcek, Phys. Rev. D 75, 124014 (2007).

[407] S. M. Carroll, I. Sawicki, A. Silvestri and M. Trodden, New J. Phys. 8, 323 (2006).

[408] Y. S. Song, W. Hu and I. Sawicki, Phys. Rev. D 75, 044004 (2007). 
[409] R. Bean, D. Bernat, L. Pogosian, A. Silvestri and M. Trodden, Phys. Rev. D 75, 064020 (2007).

[410] T. Faulkner, M. Tegmark, E. F. Bunn and Y. Mao, Phys. Rev. D 76, 063505 (2007).

[411] L. Pogosian and A. Silvestri, Phys. Rev. D 77, 023503 (2008).

[412] V. Muller, H. J. Schmidt and A. A. Starobinsky, Phys. Lett. B 202, 198 (1988).

[413] V. Faraoni, Phys. Rev. D 70, 044037 (2004).

[414] L. Amendola, R. Gannouji, D. Polarski and S. Tsujikawa, Phys. Rev. D 75, 083504 (2007).

[415] W. Hu and I. Sawicki, Phys. Rev. D 76, 064004 (2007).

[416] A. A. Starobinsky, JETP Lett. 86, 157 (2007).

[417] S. Tsujikawa, Phys. Rev. D 77, 023507 (2008).

[418] S. A. Appleby and R. A. Battye, Phys. Lett. B 654, 7 (2007).

[419] E. V. Linder, Phys. Rev. D 80, 123528 (2009).

[420] B. Li and J. D. Barrow, Phys. Rev. D 75, 084010 (2007).

[421] R. Gannouji, B. Moraes and D. Polarski, JCAP 0902, 034 (2009).

[422] D. F. Torres, Phys. Rev. D 66, 043522 (2002).

[423] B. Boisseau, G. Esposito-Farese, D. Polarski and A. A. Starobinsky, Phys. Rev. Lett. 85, 2236 (2000).

[424] H. Motohashi, A. A. Starobinsky and J. Yokoyama, arXiv:1002.1141 [astro-ph.CO].

[425] K. Bamba, C. Q. Geng, S. Nojiri and S. D. Odintsov, Phys. Rev. D 79, 083014 (2009).

[426] K. Bamba, arXiv:0909.2991 [astro-ph.CO].

[427] A. Dev, D. Jain, S. Jhingan, S. Nojiri, M. Sami and I. Thongkool, Phys. Rev. D 78, 083515 (2008).

[428] M. Martinelli, A. Melchiorri and L. Amendola, Phys. Rev. D 79, 123516 (2009).

[429] V. F. Cardone, A. Diaferio and S. Camera, arXiv:0907.4689 [astro-ph.CO].

[430] A. Ali, R. Gannouji, M. Sami and A. A. Sen, arXiv:1001.5384 [astro-ph.CO].

[431] J. c. Hwang and H. r. Noh, Phys. Rev. D 65, 023512 (2002).

[432] J. c. Hwang and H. Noh, Phys. Rev. D 71, 063536 (2005).

[433] A. De Felice and S. Tsujikawa, arXiv:1002.4928 [gr-qc].

[434] S. Tsujikawa, Phys. Rev. D 76, 023514 (2007).

[435] S. Tsujikawa, K. Uddin and R. Tavakol, Phys. Rev. D 77, 043007 (2008).

[436] S. A. Appleby and R. A. Battye, JCAP 0805, 019 (2008).

[437] A. de la Cruz-Dombriz, A. Dobado and A. L. Maroto, Phys. Rev. Lett. 103, 179001 (2009).

[438] A. de la Cruz-Dombriz, A. Dobado and A. L. Maroto, Phys. Rev. D 77, 123515 (2008).

[439] H. Motohashi, A. A. Starobinsky and J. Yokoyama, Int. J. Mod. Phys. D 18, 1731 (2009).

[440] S. Tsujikawa, R. Gannouji, B. Moraes and D. Polarski, Phys. Rev. D 80, 084044 (2009).

[441] P. Zhang, Phys. Rev. D 73, 123504 (2006).

[442] Y. S. Song, W. Hu and I. Sawicki, Phys. Rev. D 75, 044004 (2007).

[443] Y. S. Song, H. Peiris and W. Hu, Phys. Rev. D 76, 063517 (2007).

[444] L. Amendola, M. Kunz and D. Sapone, JCAP 0804, 013 (2008).

[445] S. Tsujikawa and T. Tatekawa, Phys. Lett. B 665, 325 (2008).

[446] F. Schmidt, Phys. Rev. D 78, 043002 (2008).

[447] A. Borisov and B. Jain, Phys. Rev. D 79, 103506 (2009).

[448] T. Narikawa and K. Yamamoto, Phys. Rev. D 81, 043528 (2010).

[449] H. F. Stabenau and B. Jain, Phys. Rev. D 74, 084007 (2006).

[450] W. Hu and I. Sawicki, Phys. Rev. D 76, 104043 (2007).

[451] H. Oyaizu, Phys. Rev. D 78, 123523 (2008).

[452] H. Oyaizu, M. Lima and W. Hu, Phys. Rev. D 78, 123524 (2008).

[453] F. Schmidt, M. V. Lima, H. Oyaizu and W. Hu, Phys. Rev. D 79, 083518 (2009).

[454] I. Laszlo and R. Bean, Phys. Rev. D 77, 024048 (2008).

[455] T. Tatekawa and S. Tsujikawa, JCAP 0809, 009 (2008).

[456] K. Koyama, A. Taruya and T. Hiramatsu, Phys. Rev. D 79, 123512 (2009).

[457] F. Schmidt, A. Vikhlinin and W. Hu, Phys. Rev. D 80, 083505 (2009).

[458] K. i. Maeda, Phys. Rev. D 39, 3159 (1989).

[459] S. Capozziello and S. Tsujikawa, Phys. Rev. D 77, 107501 (2008).

[460] A. V. Frolov, Phys. Rev. Lett. 101, 061103 (2008).

[461] S. Appleby, R. Battye and A. Starobinsky, arXiv:0909.1737 [astro-ph.CO].

[462] V. Miranda, S. E. Joras, I. Waga and M. Quartin, Phys. Rev. Lett. 102, 221101 (2009).

[463] I. Thongkool, M. Sami, R. Gannouji and S. Jhingan, Phys. Rev. D 80, 043523 (2009).

[464] A. de la Cruz-Dombriz, A. Dobado and A. L. Maroto, Phys. Rev. Lett. 103, 179001 (2009).

[465] T. Kobayashi and K. i. Maeda, Phys. Rev. D 78, 064019 (2008).

[466] T. Kobayashi and K. i. Maeda, Phys. Rev. D 79, 024009 (2009).

[467] S. Tsujikawa, T. Tamaki and R. Tavakol, JCAP 0905, 020 (2009).

[468] E. Babichev and D. Langlois, Phys. Rev. D 80, 121501 (2009).

[469] A. Upadhye and W. Hu, Phys. Rev. D 80, 064002 (2009).

[470] E. Babichev and D. Langlois, arXiv:0911.1297 [gr-qc].

[471] A. Palatini, Rend. Circ. Mat. Palermo 43, 203 (1919).

[472] M. Ferraris, M. Francaviglia and I. Volovich, Class. Quant. Grav. 11, 1505 (1994). 
[473] D. N. Vollick, Phys. Rev. D 68, 063510 (2003).

[474] D. N. Vollick, Class. Quant. Grav. 21, 3813 (2004).

[475] E. E. Flanagan, Class. Quant. Grav. 21, 417 (2003).

[476] E. E. Flanagan, Phys. Rev. Lett. 92, 071101 (2004).

[477] E. E. Flanagan, Class. Quant. Grav. 21, 3817 (2004).

[478] F. W. Hehl and G. D. Kerling, Gen. Rel. Grav. 9, 691 (1978).

[479] T. P. Sotiriou and S. Liberati, Annals Phys. 322, 935 (2007).

[480] T. P. Sotiriou and S. Liberati, J. Phys. Conf. Ser. 68, 012022 (2007).

[481] S. Capozziello, R. Cianci, C. Stornaiolo and S. Vignolo, Class. Quant. Grav. 24, 6417 (2007).

[482] T. P. Sotiriou and V. Faraoni, arXiv:0805.1726 [gr-qc].

[483] X. Meng and P. Wang, Class. Quant. Grav. 20, 4949 (2003).

[484] X. Meng and P. Wang, Class. Quant. Grav. 21, 951 (2004).

[485] X. H. Meng and P. Wang, Phys. Lett. B 584, 1 (2004).

[486] T. P. Sotiriou, Class. Quant. Grav. 23, 1253 (2006).

[487] T. P. Sotiriou, Phys. Rev. D 73, 063515 (2006).

[488] M. Amarzguioui, O. Elgaroy, D. F. Mota and T. Multamaki, Astron. Astrophys. 454, 707 (2006).

[489] S. Fay, R. Tavakol and S. Tsujikawa, Phys. Rev. D 75, 063509 (2007).

[490] T. Koivisto, Phys. Rev. D 73, 083517 (2006).

[491] B. Li and M. C. Chu, Phys. Rev. D 74, 104010 (2006).

[492] B. Li, K. C. Chan and M. C. Chu, Phys. Rev. D 76, 024002 (2007).

[493] A. Iglesias, N. Kaloper, A. Padilla and M. Park, Phys. Rev. D 76, 104001 (2007).

[494] G. J. Olmo, Phys. Rev. Lett. 98, 061101 (2007).

[495] G. J. Olmo, Phys. Rev. D 77, 084021 (2008).

[496] G. J. Olmo, arXiv:0910.3734 [gr-qc].

[497] E. Barausse, T. P. Sotiriou and J. C. Miller, Class. Quant. Grav. 25, 105008 (2008).

[498] E. Barausse, T. P. Sotiriou and J. C. Miller, Class. Quant. Grav. 25, 062001 (2008).

[499] G. J. Olmo and P. Singh, JCAP 0901, 030 (2009).

[500] C. Barragan, G. J. Olmo and H. Sanchis-Alepuz, Phys. Rev. D 80, 024016 (2009).

[501] V. Reijonen, arXiv:0912.0825 [gr-qc].

[502] S. M. Carroll, A. De Felice, V. Duvvuri, D. A. Easson, M. Trodden and M. S. Turner, Phys. Rev. D 71, 063513 (2005).

[503] O. Mena, J. Santiago and J. Weller, Phys. Rev. Lett. 96, 041103 (2006).

[504] A. Nunez and S. Solganik, Phys. Lett. B 608, 189 (2005).

[505] G. Calcagni, S. Tsujikawa and M. Sami, Class. Quant. Grav. 22, 3977 (2005).

[506] A. De Felice, M. Hindmarsh and M. Trodden, JCAP 0608, 005 (2006).

[507] G. Calcagni, B. de Carlos and A. De Felice, Nucl. Phys. B 752, 404 (2006).

[508] S. Nojiri, S. D. Odintsov and M. Sasaki, Phys. Rev. D 71, 123509 (2005).

[509] T. Koivisto and D. F. Mota, Phys. Lett. B 644, 104 (2007).

[510] S. Tsujikawa and M. Sami, JCAP 0701, 006 (2007).

[511] T. Koivisto and D. F. Mota, Phys. Rev. D 75, 023518 (2007).

[512] B. M. Leith and I. P. Neupane, JCAP 0705, 019 (2007).

[513] Z. K. Guo, N. Ohta and S. Tsujikawa, Phys. Rev. D 75, 023520 (2007).

[514] L. Amendola, C. Charmousis and S. C. Davis, JCAP 0612, 020 (2006).

[515] S. Nojiri and S. D. Odintsov, Phys. Lett. B 631, 1 (2005).

[516] B. Li, J. D. Barrow and D. F. Mota, Phys. Rev. D 76, 044027 (2007).

[517] G. Cognola, E. Elizalde, S. Nojiri, S. Odintsov and S. Zerbini, Phys. Rev. D 75, 086002 (2007).

[518] A. De Felice and M. Hindmarsh, JCAP 0706, 028 (2007).

[519] S. C. Davis, arXiv:0709.4453 [hep-th].

[520] A. De Felice and S. Tsujikawa, Phys. Lett. B 675, 1 (2009).

[521] S. Y. Zhou, E. J. Copeland and P. M. Saffin, JCAP 0907, 009 (2009).

[522] K. Uddin, J. E. Lidsey and R. Tavakol, Gen. Rel. Grav. 41, 2725 (2009).

[523] A. De Felice and S. Tsujikawa, Phys. Rev. D 80, 063516 (2009).

[524] A. De Felice, D. F. Mota and S. Tsujikawa, Phys. Rev. D 81, 023532 (2010).

[525] J. O'Hanlon, Phys. Rev. Lett. 29, 137 (1972).

[526] P. Teyssandier and Ph. Tourrenc, J. Math. Phys. 242793 (1983).

[527] J. P. Uzan, Phys. Rev. D 59, 123510 (1999).

[528] T. Chiba, Phys. Rev. D 60, 083508 (1999).

[529] N. Bartolo and M. Pietroni, Phys. Rev. D 61, 023518 (2000).

[530] F. Perrotta, C. Baccigalupi and S. Matarrese, Phys. Rev. D 61, 023507 (2000).

[531] C. Baccigalupi, S. Matarrese and F. Perrotta, Phys. Rev. D 62, 123510 (2000).

[532] A. Riazuelo and J. P. Uzan, Phys. Rev. D 66, 023525 (2002).

[533] L. Perivolaropoulos, JCAP 0510, 001 (2005).

[534] S. Nesseris and L. Perivolaropoulos, JCAP 0701, 018 (2007).

[535] J. Martin, C. Schimd and J. P. Uzan, Phys. Rev. Lett. 96, 061303 (2006).

[536] R. Gannouji, D. Polarski, A. Ranquet and A. A. Starobinsky, JCAP 0609, 016 (2006). 
[537] V. Acquaviva and L. Verde, JCAP 0712, 001 (2007).

[538] Y. S. Song, L. Hollenstein, G. Caldera-Cabral and K. Koyama, arXiv:1001.0969 [astro-ph.CO].

[539] P. Zhang, M. Liguori, R. Bean and S. Dodelson, Phys. Rev. Lett. 99, 141302 (2007).

[540] S. Wang, L. Hui, M. May and Z. Haiman, Phys. Rev. D 76, 063503 (2007).

[541] B. Jain and P. Zhang, Phys. Rev. D 78, 063503 (2008).

[542] S. F. Daniel, R. R. Caldwell, A. Cooray and A. Melchiorri, Phys. Rev. D 77, 103513 (2008).

[543] E. Bertschinger and P. Zukin, Phys. Rev. D 78, 024015 (2008).

[544] G. B. Zhao, L. Pogosian, A. Silvestri and J. Zylberberg, Phys. Rev. D 79, 083513 (2009).

[545] Y. S. Song and K. Koyama, JCAP 0901, 048 (2009).

[546] Y. S. Song and O. Dore, JCAP 0903, 025 (2009).

[547] J. Guzik, B. Jain and M. Takada, Phys. Rev. D 81, 023503 (2010).

[548] P. Brax, C. van de Bruck, A. C. Davis and D. Shaw, arXiv:0912.0462 [astro-ph.CO].

[549] S. F. Daniel, E. V. Linder, T. L. Smith, R. R. Caldwell, A. Cooray, A. Leauthaud and L. Lombriser, arXiv:1002.1962 [astro-ph.CO].

[550] R. Bean and M. Tangmatitham, arXiv:1002.4197 [astro-ph.CO].

[551] G. B. Zhao et al., arXiv:1003.0001 [astro-ph.CO].

[552] C. Deffayet, Phys. Lett. B 502 (2001), 199.

[553] C. Deffayet, G. R. Dvali and G. Gabadadze, Phys. Rev. D 65 (2002), 044023.

[554] V. Sahni and Y. Shtanov, JCAP 0311, 014 (2003).

[555] K. Hinterbichler, A. Nicolis and M. Porrati, JHEP 0909, 089 (2009).

[556] I. Sawicki and S. M. Carroll, arXiv:astro-ph/0510364.

[557] M. Fairbairn and A. Goobar, Phys. Lett. B 642, 432 (2006).

[558] R. Maartens and E. Majerotto, Phys. Rev. D 74, 023004 (2006).

[559] U. Alam and V. Sahni, Phys. Rev. D 73, 084024 (2006).

[560] Y. S. Song, I. Sawicki and W. Hu, Phys. Rev. D 75, 064003 (2007).

[561] J. Q. Xia, Phys. Rev. D 79, 103527 (2009).

[562] G. Dvali and M. S. Turner, arXiv:astro-ph/0301510.

[563] A. Lue, R. Scoccimarro and G. D. Starkman, Phys. Rev. D 69, 124015 (2004).

[564] K. Koyama and R. Maartens, JCAP 0601, 016 (2006).

[565] S. M. Carroll, I. Sawicki, A. Silvestri and M. Trodden, New J. Phys. 8, 323 (2006).

[566] K. Yamamoto, B. A. Bassett, R. C. Nichol and Y. Suto, Phys. Rev. D 74, 063525 (2006).

[567] K. Koyama and F. P. Silva, Phys. Rev. D 75, 084040 (2007).

[568] D. Huterer and E. V. Linder, Phys. Rev. D 75, 023519 (2007).

[569] H. Wei, Phys. Lett. B 664, 1 (2008).

[570] Y. Gong, M. Ishak and A. Wang, Phys. Rev. D 80, 023002 (2009).

[571] X. y. Fu, P. x. Wu and H. w. Yu, Phys. Lett. B 677, 12 (2009).

[572] A. I. Vainshtein, Phys. Lett. B 39, 393 (1972).

[573] C. Deffayet, G. R. Dvali, G. Gabadadze and A. I. Vainshtein, Phys. Rev. D 65, 044026 (2002).

[574] A. Gruzinov, New Astron. 10, 311 (2005).

[575] M. Porrati, Phys. Lett. B 534, 209 (2002).

[576] M. A. Luty, M. Porrati and R. Rattazzi, JHEP 0309, 029 (2003).

[577] A. Nicolis, R. Rattazzi and E. Trincherini, Phys. Rev. D 79, 064036 (2009).

[578] C. Deffayet, G. Esposito-Farese and A. Vikman, Phys. Rev. D 79, 084003 (2009).

[579] C. Deffayet, S. Deser and G. Esposito-Farese, Phys. Rev. D 80, 064015 (2009).

[580] F. P. Silva and K. Koyama, Phys. Rev. D 80, 121301 (2009).

[581] N. Chow and J. Khoury, Phys. Rev. D 80, 024037 (2009).

[582] T. Kobayashi, H. Tashiro and D. Suzuki, Phys. Rev. D 81, 063513 (2010).

[583] T. Kobayashi, arXiv:1003.3281 [astro-ph.CO].

[584] T. Buchert, Gen. Rel. Grav. 40, 467 (2008).

[585] R. A. Vanderveld, E. E. Flanagan and I. Wasserman, Phys. Rev. D 74, 023506 (2006).

[586] A. Paranjape and T. P. Singh, Class. Quant. Grav. 23, 6955 (2006).

[587] J. W. Moffat, JCAP 0605, 001 (2006).

[588] D. Garfinkle, Class. Quant. Grav. 23, 4811 (2006).

[589] D. J. H. Chung and A. E. Romano, Phys. Rev. D 74, 103507 (2006).

[590] H. Alnes and M. Amarzguioui, Phys. Rev. D 74, 103520 (2006).

[591] H. Alnes, M. Amarzguioui and O. Gron, JCAP 0701, 007 (2007).

[592] H. Alnes and M. Amarzguioui, Phys. Rev. D 75, 023506 (2007).

[593] T. Kai, H. Kozaki, K. i. Nakao, Y. Nambu and C. M. Yoo, Prog. Theor. Phys. 117, 229 (2007).

[594] K. Enqvist and T. Mattsson, JCAP 0702, 019 (2007).

[595] T. Biswas, R. Mansouri and A. Notari, JCAP 0712, 017 (2007).

[596] N. Brouzakis, N. Tetradis and E. Tzavara, JCAP 0702, 013 (2007).

[597] M. Tanimoto and Y. Nambu, Class. Quant. Grav. 24, 3843 (2007).

[598] J. Garcia-Bellido and T. Haugboelle, JCAP 0804, 003 (2008).

[599] J. Garcia-Bellido and T. Haugboelle, JCAP 0809, 016 (2008). 
[600] T. Clifton, P. G. Ferreira and K. Land, Phys. Rev. Lett. 101, 131302 (2008).

[601] S. Alexander, T. Biswas, A. Notari and D. Vaid, JCAP 0909, 025 (2009).

[602] C. Clarkson, T. Clifton and S. February, JCAP 0906, 025 (2009).

[603] K. Kainulainen and V. Marra, Phys. Rev. D 80, 127301 (2009).

[604] R. R. Caldwell and A. Stebbins, Phys. Rev. Lett. 100, 191302 (2008).

[605] C. Quercellini, M. Quartin and L. Amendola, Phys. Rev. Lett. 102, 151302 (2009).

[606] M. Quartin and L. Amendola, Phys. Rev. D 81, 043522 (2010).

[607] A. E. Romano and M. Sasaki, arXiv:0905.3342 [astro-ph.CO].

[608] M. N. Celerier, K. Bolejko, A. Krasinski and C. Hellaby, arXiv:0906.0905 [astro-ph.CO].

[609] P. Dunsby, N. Goheer, B. Osano and J. P. Uzan, arXiv:1002.2397 [astro-ph.CO].

[610] K. Saito, A. Ishibashi and H. Kodama, arXiv:1002.3855 [astro-ph.CO].

[611] F. Piazza, arXiv:0904.4299 [hep-th].

[612] F. Piazza, New J. Phys. 11, 113050 (2009).

[613] S. Nesseris, F. Piazza and S. Tsujikawa, arXiv:0910.3949 [astro-ph.CO].

[614] C. M. Hirata and U. Seljak, Phys. Rev. D 72, 083501 (2005).

[615] P. Martineau and R. H. Brandenberger, Phys. Rev. D 72, 023507 (2005).

[616] A. Ishibashi and R. M. Wald, Class. Quant. Grav. 23, 235 (2006).

[617] T. Buchert, Class. Quant. Grav. 23, 817 (2006).

[618] T. Buchert, J. Larena and J. M. Alimi, Class. Quant. Grav. 23, 6379 (2006).

[619] S. Rasanen, JCAP 0611, 003 (2006).

[620] V. Marra, E. W. Kolb, S. Matarrese and A. Riotto, Phys. Rev. D 76, 123004 (2007).

[621] M. Kasai, H. Asada and T. Futamase, Prog. Theor. Phys. 115, 827 (2006).

[622] M. Kasai, Prog. Theor. Phys. 117, 1067 (2007).

[623] D. L. Wiltshire, New J. Phys. 9, 377 (2007).

[624] J. Behrend, I. A. Brown and G. Robbers, JCAP 0801, 013 (2008).

[625] A. Paranjape, Phys. Rev. D 78, 063522 (2008).

[626] J. Larena, J. Alimi, T. Buchert, M. Kunz and P. S. Corasaniti, Phys. Rev. D 79, 083011 (2009).

[627] M. Gasperini, G. Marozzi and G. Veneziano, JCAP 0903, 011 (2009).

[628] E. W. Kolb, V. Marra and S. Matarrese, arXiv:0901.4566 [astro-ph.CO]. 